INAUGURAL - DISSERTATION

Untersuchung zur mikrochirurgischen Entfernung unterer Weisheitszähne mittels okklusalen Zugangs

Eun-Jin Choi 
Aus der Abteilung Mund-, Kiefer- und Gesichtschirurgie

(Prof. Dr. med. Dr. med. dent. H. Schliephake)

im Zentrum Zahn-, Mund- und Kieferheilkunde

der Medizinischen Fakultät der Universität Göttingen

\section{Untersuchung zur mikrochirurgischen Entfernung unterer Weisheitszähne mittels okklusalen Zugangs}

\section{INAUGURAL - DISSERTATION}

zur Erlangung des Doktorgrades für Zahnheilkunde

der Medizinischen Fakultät der Georg-August-Universität zu Göttingen

vorgelegt von

Eun-Jin Choi

aus

Aachen

Göttingen 2012 
Dekan: Prof. Dr. med. M. P. Schön

I. Berichterstatter: Prof. Dr. med. Dr. med. dent. W. Engelke

II. Berichterstatter: Prof. Dr. med. Hommerich

III. Berichterstatter: Prof. Dr. med. Oppermann

Tag der mündlichen Prüfung: 27.08.2013 
Die vorliegende Promotionsschrift war die Grundlage für folgende Veröffentlichung:

Engelke W, Bierbaum J, Choi EJ (2011):

Die mikrochirurgische Entfernung unterer dritter Molaren über einen okklusalen Zugang.

Dtsch Zahnärztl Z $\underline{66}, 415-423$ 


\section{Inhaltsverzeichnis}

1.

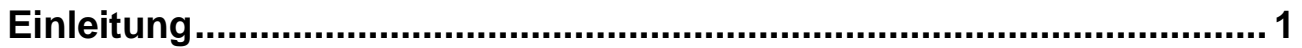

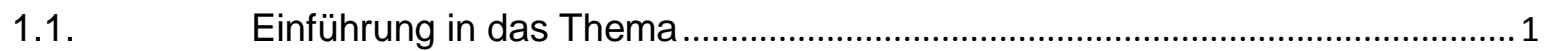

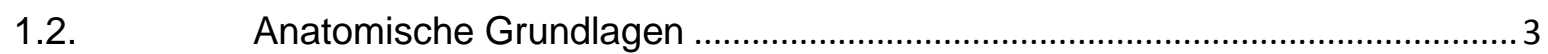

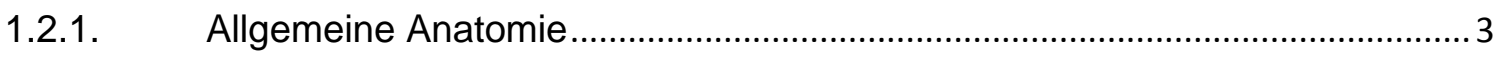

1.2.2. Entwicklung des Weisheitszahnes ..................................................................... 7

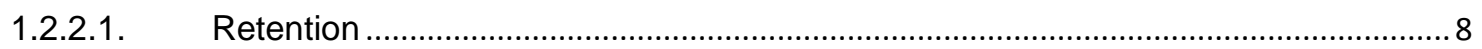

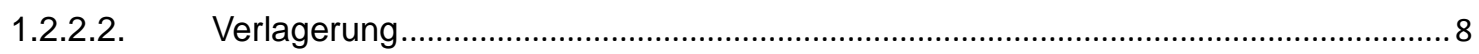

1.3. Pathologie und Klinik des Weisheitszahnes ..............................................11

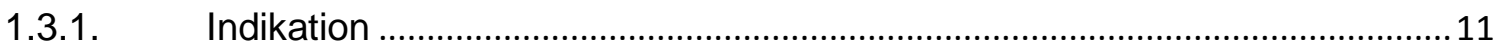

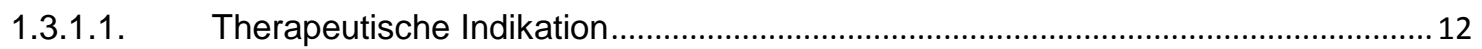

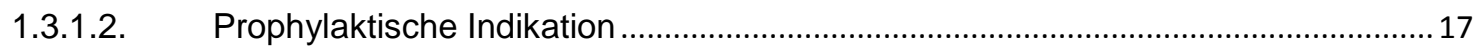

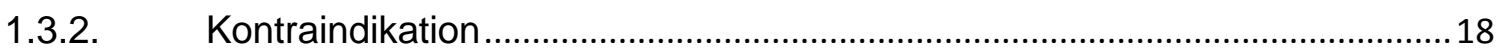

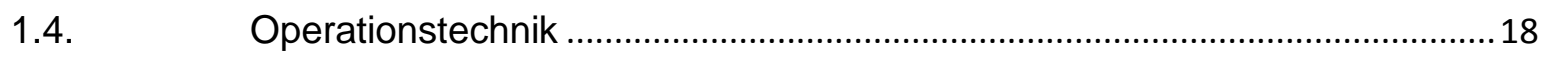

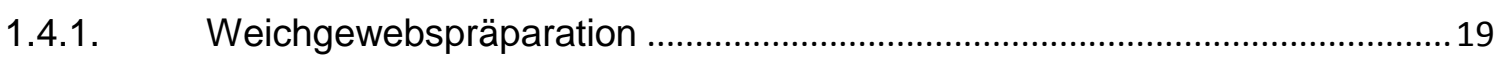

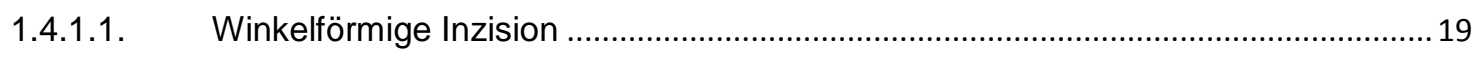

1.4.1.2. Spaltlappen-Inzision (Partial-Thickness Flap) ....................................................... 20

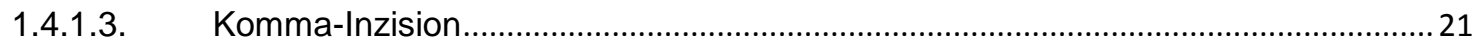

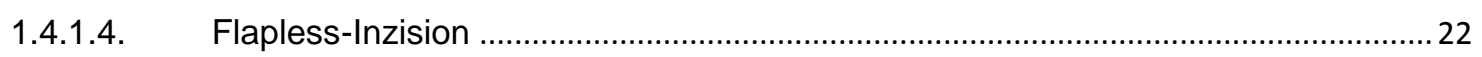

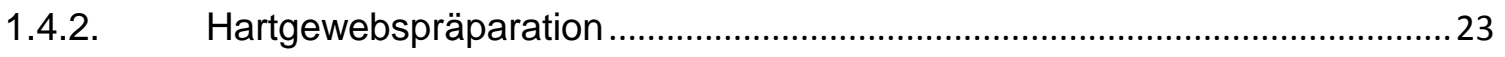

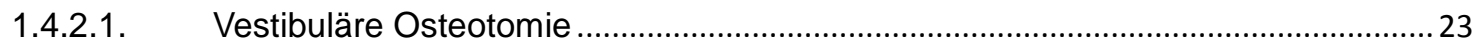

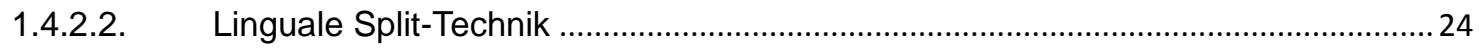

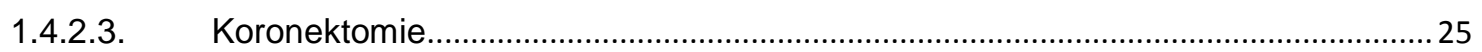

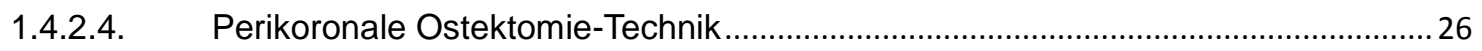

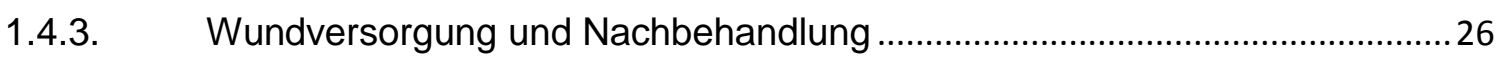

1.5. Literaturübersicht zur Auswertung der Knochendefektsituation ....................28

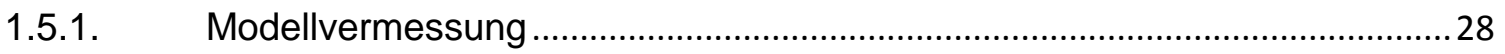

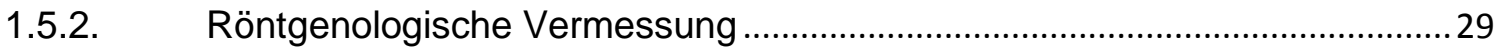

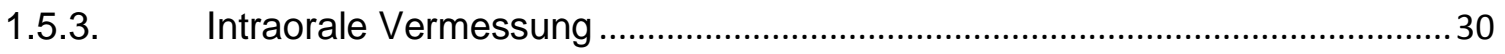

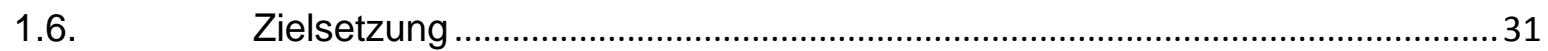

2. Material und Methode........................................................................ 32

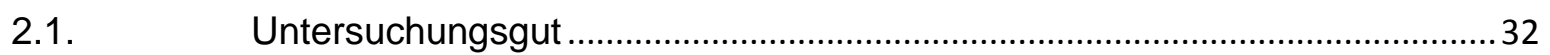

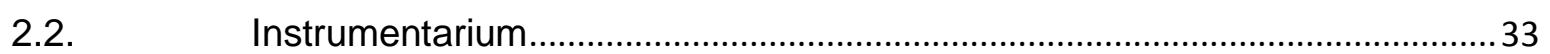

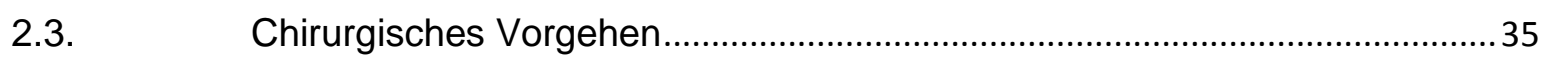

2.4. Dokumentation der Röntgenbefunde ............................................................ 42 


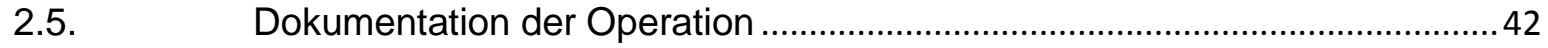

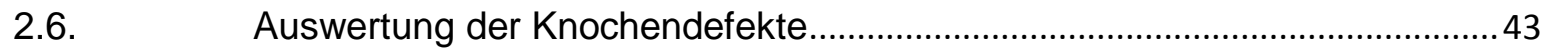

2.6.1. Präoperative Vermessung des Röntgenbildes ................................................. 43

2.6.2. Intraoperative Vermessung der Alveolenwand ................................................ 45

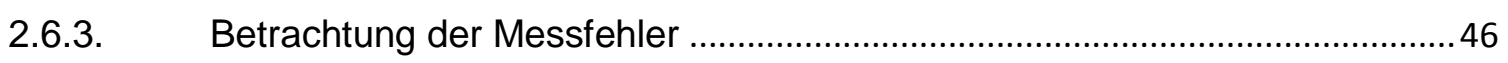

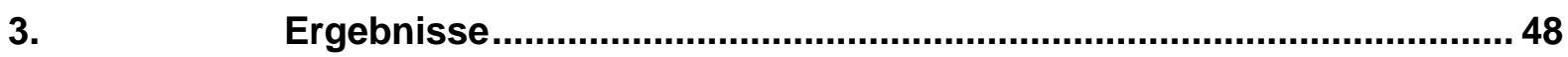

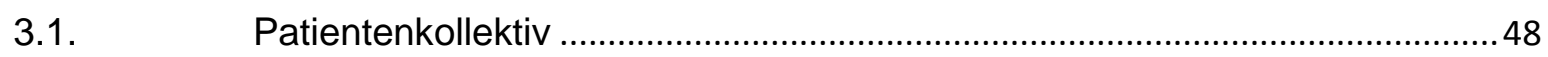

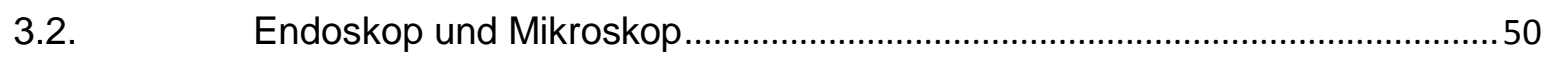

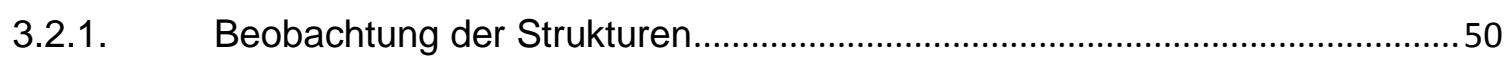

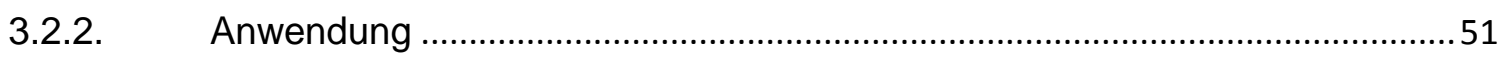

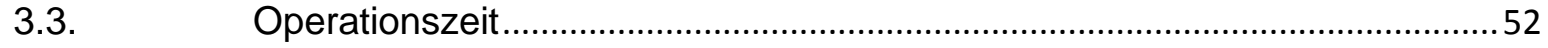

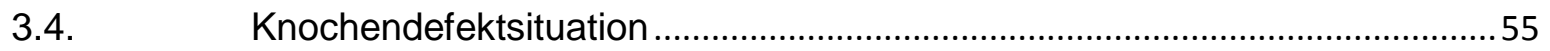

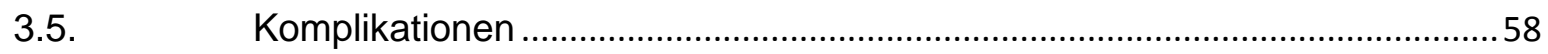

4.

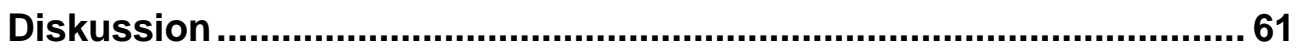

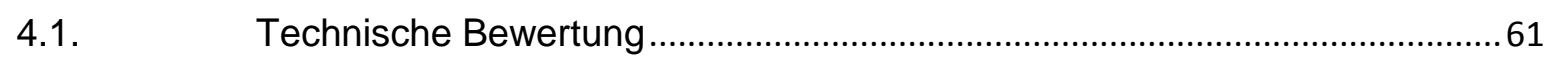

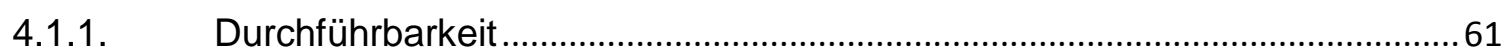

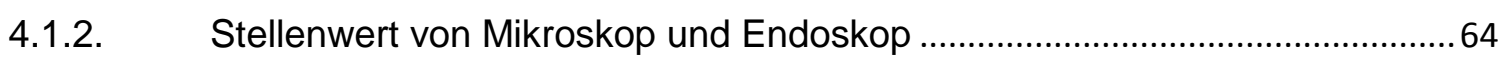

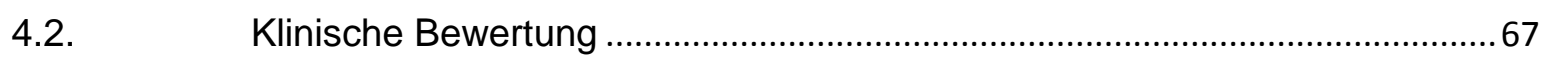

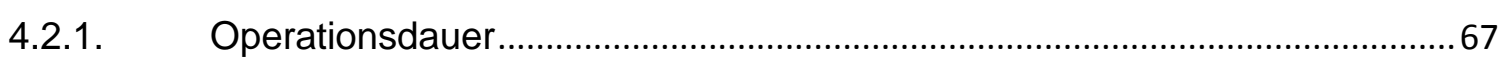

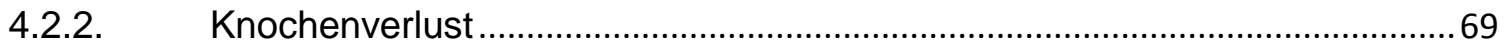

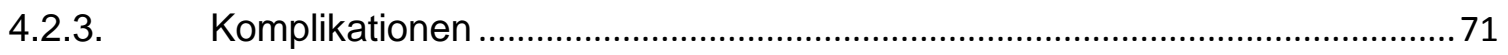

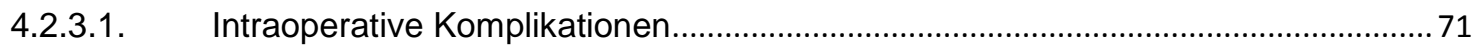

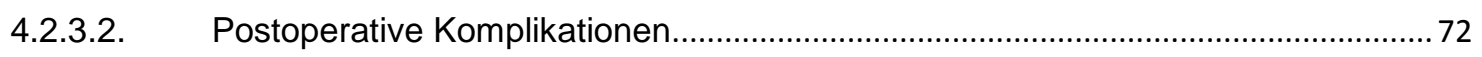

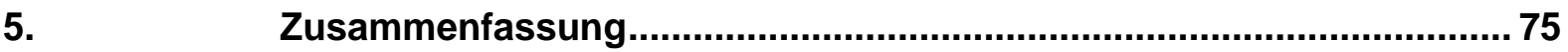

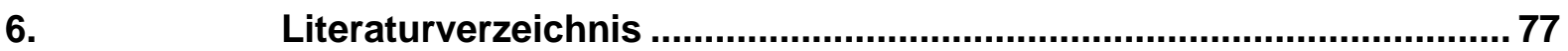

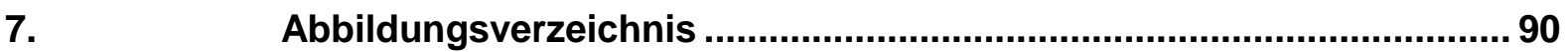

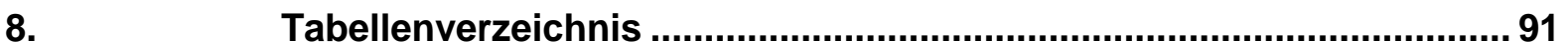

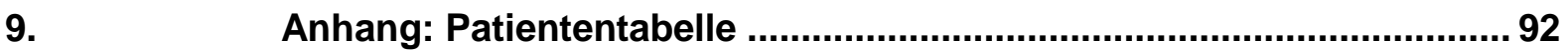




\section{Einleitung}

\subsection{Einführung in das Thema}

Den dritten Molaren, umgangssprachlich auch Weisheitszahn genannt, haben $90 \%$ der Bevölkerung und 33\% mit mindestens einem impaktierten Zahn (Schersten et al. 1989). Die Tatsache, dass bei dieser Prävalenz die operative Weisheitszahnentfernung einer der häufigsten zahnärztlichchirurgischen Eingriffe ist (Sands et al. 1993), ist nicht erstaunlich. Obwohl die operative Weisheitszahnentfernung so häufig durchgeführt wird, kann man aus zahlreichen wissenschaftlichen Studien entnehmen, dass Hartwie auch Weichgewebsmanipulationen die Beschwerderate der Patienten hinsichtlich Schmerz, Schwellung und Trismus steigen lassen. Zahlreiche Faktoren wie z. B. anatomische Unterkieferstrukturen, Retentionsart und Verlagerungsart des Weisheitszahnes, Alter des Patienten etc. erschweren zusätzlich die Entfernung von Unterkieferweisheitszähnen (Bruce et al. 1980; Chuang et al. 2007). Teilweise treten auch intraund/oder postoperative Komplikationen auf. Reich et al. (2005) konnten in ihrer Studie eine allgemeine intraoperative Komplikationsrate von 5,8\% nachweisen. In anderen Studien konnten postoperative Komplikationsraten von 8,4\% (Van Gool et al. 1977), 8,9\% (Barbosa-Rebellato et al. 2011) bis $18,9 \%$ (Chuang et al. 2007) nachgewiesen werden. Strietzel und Reichart (2002) gaben die postoperativen Komplikationen mit $32 \%$ postoperativen Schmerzen, 28\% Schwellungen, 18\% Kieferklemme, 13\% Sensibilitätsstörungen und $9 \%$ Sonstigem an.

Da die Morbidität nach Osteotomie noch immer ein erhebliches Problem darstellt, ist die Entwicklung von Methoden mit möglichst geringem intraoperativen Trauma und postoperativen Komplikationen erforderlich.

Die Prinzipien der Oralchirurgie bekommen diesbezüglich einen neuen Trend in Richtung der minimal-invasiven, atraumatischen Operation. In den letzten Jahren wurden verschiedene Techniken vorgestellt, die dieses ermöglichen. Die Odontosektion sowie die Empfehlung der partiellen Entfernung von Kronen dritter Molaren sind ein Beispiel hierfür. 
Im Vergleich zum Gebiet der Weisheitszahnoperation wurden bereits in den letzten Jahren im Gebiet der Implantologie Operationstechniken entwickelt, die weniger intraoperative Traumata und Komplikationen hinterließen. Eine Technik davon wurde von Choi (2010) sowie Choi und Engelke (2009) ausführlich dargestellt. Es handelt sich um eine minimalinvasive Operationstechnik, die einen Verzicht auf den Mukoperiostlappen beinhaltet. Die Vorteile, die sich in Tierexperimenten zeigten, waren hinsichtlich der Reduktion von Weichgewebstraumata, des besseren Erhaltes der Vaskularisation von Knochengewebe und struktureller Vorteile für den Zustand der periimplantären Gingiva enorm (Choi 2010).

Da diese Vorteile auch bei Weisheitszahnoperationen hilfreich sein könnten, wäre die Überlegung naheliegend, das Weichgewebstrauma bei Weisheitszahnoperationen durch Mini-Inzisionen analog zur Implantologie zu reduzieren. Diese Idee wurde bislang nicht verfolgt, da bei der Weisheitszahnoperation die Übersichtlichkeit des Operationsfeldes besonders wichtig für den Operateur war (Pederson 1988). Dieses Problem der Übersicht konnte durch die Anwendung von visuellen Hilfsmitteln neu bearbeitet werden.

In der Oralchirurgie wird analog zur Allgemeinchirurgie die Anwendung von Endoskopen immer mehr bevorzugt. Der Einsatz wird in verschiedenen Gebieten wie z. B. in der Implantologie und Sinusbodenaugmentation belegt (Engelke und Capobianco 2005; Schleier et al. 2008). Juodzbalys et al. (2008) haben in einer Untersuchung zur Bewertung der Stützimmersionsendoskopie gezeigt, dass sie als unterstützendes Instrument zur Beurteilung der Morphologie und der knöchernen Bedingungen der Extraktionsalveole ohne Lappenbildung eingesetzt werden kann.

Auf der Basis der Erfahrung mit der Stützimmersionsendoskopie (Engelke 2002) mit implantologischer Indikation wurde ein Verfahren entwickelt, das die Entfernung impaktierter dritter unterer Molaren über einen okklusalen Zugang erlaubt und somit eine laterale Osteotomie vermeidet. 


\subsection{Anatomische Grundlagen}

\subsubsection{Allgemeine Anatomie}

\section{Der Unterkiefer}

Der Unterkiefer ist ein Teil des Gesichtsschädels und wird in den horizontalen Anteil "Körper“ (Corpus mandibulae) und den zum Kiefergelenk aufsteigenden Anteil „Ast“ (Ramus mandibulae) eingeteilt. Am aufsteigenden Ast befindet sich ein Muskelfortsatz (Processus coronoideus) für den Ansatz des Musculus temporalis und der Gelenkfortsatz (Processus condylaris) mit dem Kiefergelenksköpfchen (Caput mandibulae), der das Kiefergelenk bildet.

\section{Der Alveolarknochen}

Im Alveolarfortsatz (Pars alveolaris), der zum horizontalen Anteil des Unterkiefers gehört, sind die Zähne in den Zahnfächern (Alveoli dentales) verankert. Dieser Anteil von Kieferknochen ist in der Entwicklung wie auch in der Rückbildung abhängig von den Zähnen (Schroeder 2000). In Abhängigkeit der unterschiedlichen Größe, der Form und der Stellung der Zähne im Kieferknochen ergeben sich Unterschiede in der Morphologie des Alveolarfortsatzes.

Der Aufbau dieser Knochenstruktur wird in drei Anteile unterteilt. Die äußerste Schicht, die Kortikalis, wird als eine von Knochenhaut (Periost) bedeckte Knochenplatte beschrieben und ist unterschiedlich dick.

Die zweite Schicht ist die Spongiosa, die aus zarten, netzförmig angeordneten Knochenbälkchen besteht, zwischen denen sich Knochenmarksräume befinden (Schroeder 2000). Die letzte Schicht, die innere Knochenplatte, die die Alveolenwände bildet, besitzt den Charakter eines feinlöchrigen Siebes und ist somit eine sehr poröse Kortikalis, die auch Lamina cribriformis genannt wird. Im koronalen und apikalen Bereich der Alveolen sind zahlreiche Foramina vorhanden, die als Volkmann-Kanäle bezeichnet werden (Schroeder 2000). Diese Kanäle verbinden das Desmodont mit den Knochenmarksräumen und werden von Blut- und 
Lymphgefäßen sowie von Nervenfasern durchzogen (Birn 1966). Am Eingang der Alveolenfächer (Limbus alveolaris) geht die äußere Kortikalisschicht in die Lamina cribriformis über. Die Struktur der Kortikalis und der Knochenbälkchen der Spongiosa besteht aus Havers-Systemen (Osteomen) und Lamellen (McMillan 1924). Im Vergleich zum Oberkiefer ist die Kortikalis im Unterkiefer generell stärker ausgeprägt und dicker, aber geringer vaskularisiert. Im Unterkiefer selbst ist die orale Kortikalis dicker als die vestibuläre (Schroeder 1997). Im Gegensatz dazu ist der Spongiosaanteil im Oberkiefer größer als im Unterkiefer. Diese unterschiedliche Knochenstruktur führt $\mathrm{zu}$ einem erschwerten operativen Eingriff im Unterkiefer im Vergleich zum Oberkiefer.

Zur Funktion der Alveolarfortsätze gehört die Verankerung der Zähne und die Aufnahme und Verteilung jener Kräfte, die als Druck durch intermittierenden Zahnkontakt beim Kauen, Schlucken, Sprechen und bei parafunktionalem Stress (Knirschen, Pressen) ausgelöst werden (Schroeder 2000).

\section{Das Periost/Endost}

Der Knochen wird an seiner Oberfläche von Bindegewebe bedeckt, was man im äußeren Bereich als Periost bezeichnet und im inneren Bereich als Endost. Das Periost besteht aus zwei Bindegewebsschichten, dem äußeren Stratum fibrosum und dem am Knochen anliegenden Stratum osteogenicum. Das Stratum fibrosum ist eine sehr faserreiche Schicht mit Fibroblasten und sogenannten Sharpey-Fasern, die in die Knochenmatrix eintreten und das Periost am Knochen befestigen. Das Stratum osteogenicum ist hingegen eine sehr zell-, nerven- und gefäßreiche Schicht mit Fibroblasten und Knochenvorläuferzellen. Das Periost trägt wesentlich zur Ernährung und Erhaltung des Knochens bei.

Das Endost besteht aus abgeflachten Bindegewebszellen, kleidet den Knochen sowie die Knochenkanälchen von innen aus und bedeckt auch die Spongiosabälkchen. Gemeinsam mit dem Periost bildet das Endost neuen Knochen (Junqueira et al. 1996). 


\section{Die Nerven}

Der vom Nervus mandibularis abzweigende Nervus alveolaris inferior verläuft an der Innenseite des Ramus mandibulae nach kaudal zum Unterkieferloch (Foramen mandibulae) und entlässt kurz vorher den Nervus mylohyoideus, der weiter zum Musculus mylohyoideus und zum vorderen Bauch des Musculus digastricus zieht. Der Nervus alveolaris inferior folgt $a b$ dem Foramen mandibulae dem Verlauf des Unterkieferkanals (Canalis mandibulae) bis zum Kinnloch (Foramen mentale), den er als Nervus mentalis für die Versorgung der Haut des Kinns und der Haut und Schleimhaut der Unterlippe verlässt. Für die Innervation der Zähne und des Zahnfleisches trennen sich die Rami dentales inferiores und die Rami gingivales inferiores im Unterkieferkanal von dem Nervus alveolaris inferior. Der Canalis mandibulae verläuft oft in unmittelbarer Nähe des unteren dritten Molaren, sodass der Nervus alveolaris inferior bei operativen Eingriffen im Rahmen einer Weisheitszahnentfernung manipuliert werden und es zu einer postoperativen Sensibilitätsstörung kommen kann. Diese Komplikation wurde schon häufig untersucht, wobei eine postoperative Sensibilitäts-störung von $0 \%$ (Bell 2004), 3,6\% (Gülicher und Gerlach 2001) bis zu 8,4\% (Lopes et al. 1995) ermittelt worden ist. Eine dauerhafte Schädigung des Nervus alveolaris inferior ist jedoch mit 0,014\% (Alling 1986) und 0,91\% (Carmichael und McGowan 1992; Gülicher und Gerlach 2001) im Vergleich zu Irritationen relativ gering.

Aber nicht nur der Nervus alveolaris inferior, sondern auch der Nervus lingualis, der ebenfalls vom Nervus mandibularis stammt, ist für operative Eingriffe eine wichtige anatomische Struktur. Der Nervus lingualis gelangt ebenfalls an der Innenseite des Ramus mandibulae nach kaudal, zieht dabei aber vor dem Nervus alveolaris inferior nach unten und begleitet inn nicht im Canalis mandibulae. Unterhalb der mandibulären Befestigung des Musculus constrictor pharyngis superior und der Raphe pterygomandibularis wendet sich der Nervus lingualis bogenförmig nach anterior und bekommt so häufig Kontakt zum medialen Periost im Wurzelbereich 
der hinteren Unterkiefermolaren (Williams 1995). Im weiteren Verlauf zieht er nach vorn in den Mundboden und verzweigt sich in seine Rami linguales, welche die vorderen zwei Drittel der Zunge sensibel versorgen.

Die Lage des Nervus lingualis in der unteren Weisheitszahnregion wurde von Kiesselbach und Chamberlain im Jahre 1984 beschrieben. In der horizontalen bis zur lingualen Alveolarwand ist der Nervus lingualis nur ca. $0,58 \mathrm{~mm}$ entfernt (mit einer Streuung von $\pm 0,9 \mathrm{~mm}$ ) und in der vertikalen bis zum Alveolarkamm ca. 2,28 mm (mit einer Streuung von $\pm 1,96 \mathrm{~mm}$ ). Das bedeutet, dass der Nerv Kontakt am Alveolarknochen aufweisen kann oder auch auf dem Knochen, gegebenenfalls über dem Knochen liegt. Aufgrund dieser Variabilität bezüglich seines Verlaufs am Alveolarknochen entlang, ist die Gefahr einer Nervenschädigung höher als bei dem Nervus alveolaris inferior. Die allgemeine Häufigkeit postoperativer Sensibilitätsstörungen nach operativer Entfernung der unteren Weisheitszähne im Innervationsgebiet des Nervus lingualis liegt zwischen $0 \%$ (Chiapasco et al. 1993), 2,1\% (Gülicher und Gerlach 2001) bis 23\% (Middlehurst et al. 1988). Betrachtet man jedoch die zahlreich zu diesem Thema veröffentlichten Arbeiten, so zeigt nur ein geringer Anteil von 0,008\% (Alling 1986), 0,37\% (Gülicher und Gerlach 2001) und 0,6\% (Carmichael und McGowan 1992) der beobachteten Nerventraumatisierungen, dauerhafte Folgen.

\section{Der Mundboden (Diaphragma oris)}

Der Mundboden spannt sich zwischen dem bogenförmig verlaufenden Unterkieferkörper aus und ist die untere Begrenzung der Mundhöhle. Er wird aus den suprahyalen Muskeln gebildet. Der Musculus mylohyoideus bildet mit seinen beiden Anteilen rechts und links den vorderen Mundboden. Beide Muskeln setzen am Unterkiefer an und verbinden sich in der Mitte über eine bindegewebige Raphe mylohyoideus zu einer Muskelplatte. Die Ansatzlinie des Musculus mylohyoideus ist in manchen Fällen unterbrochen und gestuft, sodass sich Entzündungsprozesse von einer Loge in die andere ausbreiten können. Der Musculus geniohyoideus 
bildet den Mundboden über dem Musculus mylohyoideus und der Musculus digastricus venter anterior darunter. Hinten wird der Mundboden von dem Musculus digastricus venter posterior und von dem Musculus stylohyoideus gebildet. All diese Muskeln inserieren am Zungenbein (Os hyoideum).

Die Funktion des Mundbodens besteht darin, den Inhalt der Mundhöhle gegen die Schwerkraft abzusichern, das Zungenbein und den Larynx beim Schluckakt nach kranial zu ziehen und den Unterkiefer bei der Kieferöffnung herabzuziehen (wobei dann das Zungenbein von den infrahyoidalen Muskeln fixiert wird). Ihre Innervation ist komplex, da sie embryonal von verschiedenen Anlagen abstammen. So wird der Musculus mylohyoideus sowie der Musculus digastricus venter anterior vom Nervus mylohyoideus (Mandibularis-Nerv), der Musculus geniohyoideus vom Nervus hypoglossus versorgt und die hinteren oberen Zungenbeinmuskeln vom Nervus facialis innerviert.

\subsubsection{Entwicklung des Weisheitszahnes}

Weisheitszähne sind die einzigen Zähne, die sich erst nach der Geburt vollständig entwickeln. Die Entwicklung aller anderen Zähne beginnt in der Embryonalzeit. Sie wurden aus der ektodermalen Zahnleiste und dem Mesenchym gebildet (Drews 2006). Auch die Knospe des bleibenden Zahnes entwickelt sich neben dem Milchzahn in der Embryonalzeit und bleibt in der mesodermalen Hülle bis zum Durchbruch eingeschlossen. Die Entwicklung des Weisheitszahnes wird nicht allein von der ektodermalen Zahnleiste ausgelöst, sondern beginnt erst nach Interaktion mit dem Kiefermesenchym der kranialen Neuralleiste (Ten Cate 1998). Dies geschieht in den ersten 5 Jahren nach der Geburt mit der Größenentwicklung des Kiefers. Wenn diese beiden Komponenten nicht aufeinander stoßen, wird der Weisheitszahn nicht gebildet.

Da verschiedene Umweltfaktoren oder genetische Faktoren bis zu diesem Zeitpunkt das Wachstum des Kiefers sowie die Migration der Zahnleiste 
beeinflussen können, ist die Positionierung der Weisheitszähne bis zu diesem Zeitpunkt nicht vorgegeben. Oft stehen durch Erbanlagen Kieferund Zahngrößen nicht im richtigen Verhältnis, was zu einer Retention sowie auch Verlagerung der Weisheitszähne führen kann. Auch Krankheitsbilder, die einen Einfluss auf das Wachstum haben, können eine Ursache darstellen. Rachitis, Funktionsstörungen der Hypophyse, der Schilddrüse, des Thymus sowie Missbildungssyndrome wie LippenKiefer- Gaumen-Spalten, Dysostosis cleidocranialis oder Langdon-DownSyndrom können Entwicklungsstörungen erzeugen.

\subsubsection{Retention}

Der Begriff Retention bezeichnet eine Position des Weisheitszahnes, bei der nach Abschluss des Wurzelwachstums die Okklusionsebene nicht erreicht wird. Es gibt allgemein drei Formen der Retention, die vom Durchbruchsgrad des Zahnes abhängig sind. Komplett oder vollretinierte Zähne sind vollständig mit Mundschleimhaut bedeckt und sind so von der Mundhöhle isoliert. Bei einer teil- oder partiellen Retention spricht man von Zähnen, die nur leicht mit dem Kronenanteil in die Mundhöhle durchgebrochen sind und der restliche Kronenanteil noch von Schleimhaut oder Knochen bedeckt ist. Zuletzt gibt es die impaktierten Zähne, die komplett im Knochen eingeschlossen sind oder aufgrund benachbarter hartgewebiger Hindernisse nicht durchbrechen können. Da die unteren Weisheitszähne mit 57\% aller Zahnretentionen nicht selten davon betroffen sind (Kristen 1978), hat es eine erhebliche Bedeutung in der Indikation und zeitlichen Planung der Entfernung.

\subsubsection{Verlagerung}

Liegt eine irreguläre Durchbruchsrichtung vor oder sind die Zähne an der falschen Stelle im Kiefer, spricht man von einer Verlagerung.

Um die Verlagerungsart des Weisheitszahnes zu bestimmen, wurden von zahlreichen Autoren anhand von Hilfslinien und Hilfsstrukturen die 
Position und Beschaffenheit definiert. Winter hat im Jahre 1926 mit drei Hilfslinien, die er im Röntgenbild eingezeichnet hat, die Lage des dritten Molaren im Vergleich zum zweiten Molaren beschrieben. So konnte er verschiedene Verlagerungsformen wie vertikal, horizontal, mesial, distal und transversal angulierte Zähne definieren (Abb.1.1).

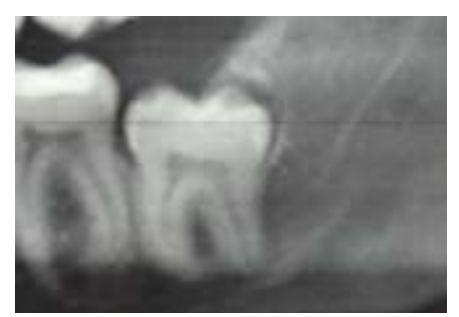

Vertikal

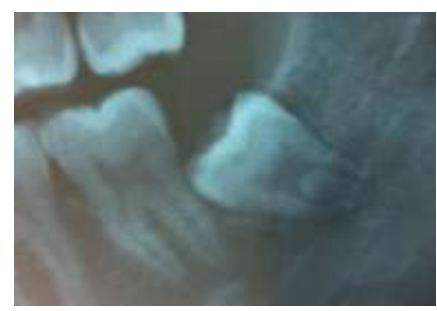

Mesioangulär

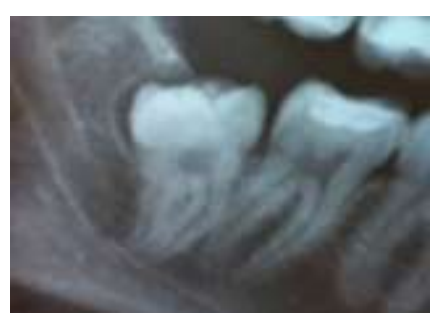

Distoangulär

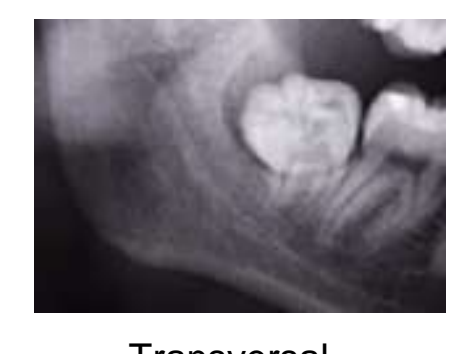

Transversal

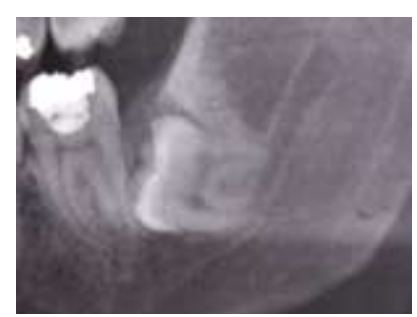

Horizontal

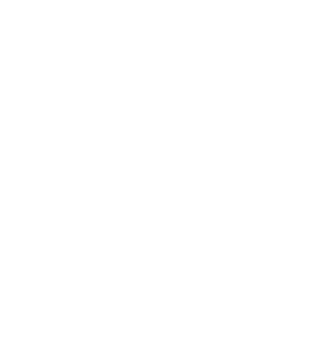

Abb.1.1: Röntgenologische Darstellung der Verlagerungsformen: die Klassifikation ist nach Winter (1926)

Pell und Gregory (1933) haben ebenfalls die Verlagerungsformen beschrieben, wobei sie die Tiefenlage im Vergleich zum zweiten Molaren wie auch das Platzangebot für den Durchbruch des dritten Molaren definierten. Die Tiefenlage wurde anhand der Schmelz-Zement-Grenze des zweiten Molaren festgestellt und in drei Stufen definiert $(A, B, C)$ (Abb.1.2). Eine weitere Klassifizierung erfolgte in der sagittalen Ebene. Durch das Platzangebot distal des zweiten Molaren bis zum aufsteigenden Ast des Unterkiefers haben Pell und Gregory (1933) weitere drei Stufen definiert (I, II, III) (Abb.1.3). 


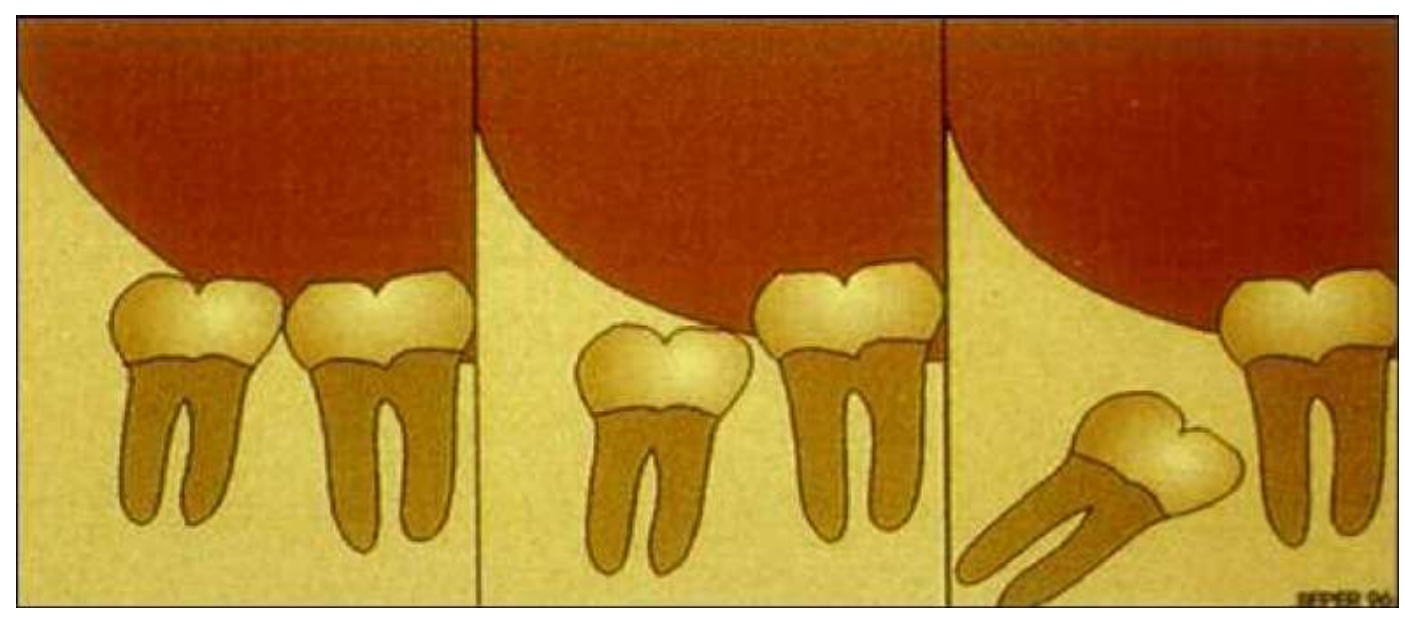

Abb.1.2: Schematische Darstellung der Pell- und Gregory-Klassifikation - nach kranio-kaudaler Lage

Level A: Krone des retinierten Zahnes liegt etwa auf der Höhe der Okklusionsebene oder sogar darüber

Level B: Die kraniale Begrenzung der Weisheitszahnkrone befindet sich kaudal der Okklusionsebene, aber kranial der Schmelz-Zement-Grenze des zweiten Molaren

Level C: Weisheitszahnkrone liegt kaudal der Schmelz-Zement-Grenze des zweiten Molaren

(Bähr 1996, S.886) 


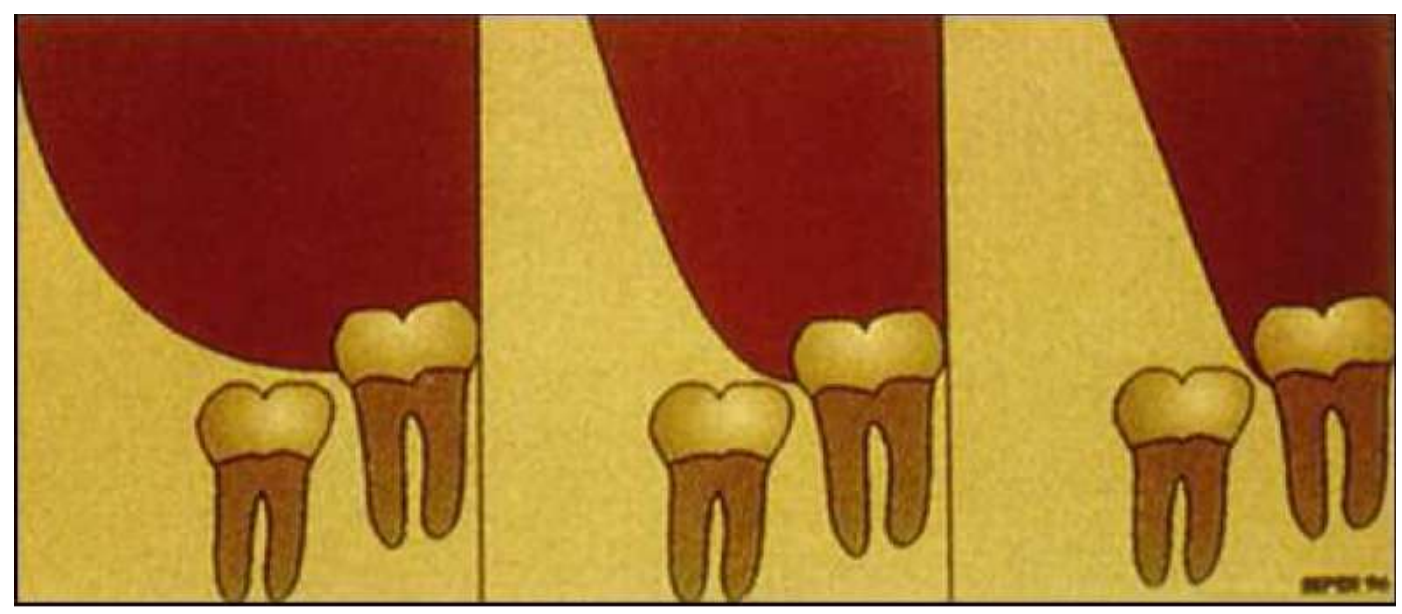

Abb.1.3: Schematische Darstellung der Pell- und Gregory-Klassifikation - nach Lagebeziehung zum aufsteigenden Unterkieferast

Klasse I: Platzangebot distal des zweiten Molaren ist so groß, dass der retinierte Weisheitszahn durchbrechen könnte

Klasse II: Der Raum distal des zweiten Molaren ist kleiner als die mesio-distale Weisheitszahnkrone

Klasse III: Die vordere Begrenzung des aufsteigenden Astes schließt direkt am zweiten Molaren an, sodass der Weisheitszahn vollständig innerhalb des Unterkieferastes liegt

(Bähr 1996, S.887)

Diese Klassifizierung gibt dem Operateur wichtige Hinweise, eine intensive Planung aufzustellen und den Schwierigkeitsgrad der Operation einzuschätzen.

\subsection{Pathologie und Klinik des Weisheitszahnes}

\subsubsection{Indikation}

In der Leitlinie „operative Entfernung von Weisheitszähnen“ (Kunkel und Wagner 2006), die bei der zahnärztlichen Zentralstelle Qualitätssicherung erschien, wurde die Indikation zwischen klinisch bzw. radiologisch symptomatischen und symptomlosen Zähnen unterschieden. Die erste Gruppe, die klinisch oder radiologisch symptomatischen Weisheitszähne, 
wurde in der Literatur weitgehend als eine Indikation beschrieben, während für die zweite Gruppe, die symptomlosen Weisheitszähne, keine evidenzbasierten Empfehlungen gegeben werden konnten (Song et al. 2000). Dennoch werden nach Literaturangaben mehr prophylaktische Entfernungen vorgenommen als therapeutische. Nach einer retrospektiven Studie nach Leesemann (2001) liegt ein Verhältnis von 85\% prophylaktisch gegenüber $15 \%$ therapeutisch bedingter Weisheitszahnentfernungen vor. Im Unterkiefer speziell wurde von Neumann (2003) belegt, dass die Weisheitszähne zu 73,9\% aus prophylaktischen und zu $26,1 \%$ aus therapeutischen Gründen entfernt worden sind.

\subsubsection{Therapeutische Indikation}

Eine therapeutische Indikation besteht laut Otten (2002), wenn pathologische Befunde des Zahnes selbst oder seiner Umgebungsgewebe eine Erhaltung unmöglich machen oder wenn erforderliche Maßnahmen einer Behandlung von Patienten abgelehnt werden.

\section{- Dentitio difficilis}

Der Begriff „Dentitio difficilis“ bedeutet „erschwerter Zahndurchbruch“ und ist einer der häufigsten Indikationen zur operativen Weisheitszahnentfernung, da es zu einer Schlupfwinkelinfektion „Perikoronitis“ führen kann (Otten 2002). Die verlängerte Eruptionsphase des Weisheitszahnes ist häufig durch Platzmangel und schräge Durchbruchsrichtung gefährdet, sodass das die Zahnkrone umschließende Epithel keinen Anschluss an die Gingiva findet. Es entsteht eine Retentionsnische für Zelldentritus und Speisereste, die über längere Zeit mit den potentiell pathogenen Bakterien der Mundflora eine Infektion auslösen kann. Entzündungszeichen wie Rötung, Schwellung und Schmerzen sind die frühen klinischen Symptome. Im Verlauf sind auch die Lymphknoten beteiligt und die entzündliche Infiltration benachbarter Gewebe führt zur schmerzhaften Mundöffnungseinschränkung. Fieber und allgemeine Krankheitsgefühle begleiten das Geschehen. Die Therapie der Wahl ist die Beseitigung der Entzündung 
durch Drainage und Reinigung und später die operative Entfernung des Weisheitszahnes. Als Komplikation einer nicht oder unzureichend behandelten "Dentitio difficilis" kann es des Weiteren zu einer eitrig einschmelzenden Entzündung mit Ausbreitungstendenz in die parapharyngeale oder seltener in die pterygomandibuläre oder masseterico-mandibuläre Loge kommen (Otten 2002).

\section{- Pathologische Strukturen in Zusammenhang mit Zahnfollikeln}

Oft entwickeln sich Zysten aus Epithelgewebe der Zahnanlage. Follikuläre Zysten, die sich aus dem Epithel der Zahnleiste entwickeln, sind die häufigsten, die am Weisheitszahn beobachtet werden. Auch radikuläre Zysten, deren Ursprung auf entzündlicher Basis im apikalen oder lateralen Parodontium aus Malassez-Epithelresten zurückzuführen ist, können auftreten. Primordial- und Keratozysten, die ebenfalls aus dem Epithel der Zahnleiste entstehen, sind seltener bei Weisheitszähnen auffindbar. Zysten entstehen, wenn entzündliche oder traumatische Reize zu einer Epithelproliferation führen oder wenn das Epithel von sich aus proliferiert. Da bei Weisheitszähnen eine verlängerte Durchbruchszeit einen ständigen Reiz auf das Zahnsäckchen ausübt, sind diese besonders gefährdet. Die Inzidenz einer Zyste im Zusammenhang mit einem Weisheitszahn, befindet sich bei 2,3\% (Güven et al. 2000). Oft sind Zysten beim Wachstum symptomfrei und werden als Zufallsbefund im Röntgenbild entdeckt. Diese Zysten sollten vor einem erheblichen Ausmaß der Größe entfernt werden, da sie sonst dem umgebenden Gewebe durch Druckatrophie, Verdrängung oder Resorption Schaden zufügen können (Schwenzer und Ehrenfeld 2009).

Bei der operativen Entfernung wird auch der Weisheitszahn mit entfernt, damit die Rezidivgefahr möglichst klein gehalten werden kann. Zur Differenzialdiagnose sollte der Zystenbalg pathologisch untersucht werden, um sicherzustellen, dass kein tumorartiges Geschehen, welches mit 0,79\% relativ selten auftritt (Güven et al. 2000), übersehen wird. Dabei sollte man bedenken, dass follikuläre Zysten zu zystischen 
Ameloblastomen entarten können. Die Inzidenz der Ameloblastome im Zusammenhang mit den Weisheitszähnen liegt nach Regezi et al. (1978) bei $0,14 \%$ und ist somit selten.

Aber nicht nur die Ameloblastome sind eine Indikation zur notwendigen Therapie. Bei allen Geweben der Mundhöhle können grundsätzlich gutartige Tumore im Zusammenhang mit Weisheitszähnen auftreten. Eine frühzeitige Erkennung der Diagnose wie auch die nachfolgende Therapie ist sehr wichtig, um eine maligne Entartung sowie größere Defekte zu vermeiden.

\section{- Weisheitszahn, als relevante Schmerzursache}

Die "International association for the study of pain" definiert Schmerz als unangenehmes Sinnes- oder Gefühlserlebnis, das mit tatsächlicher oder potenzieller Gewebeschädigung einhergeht oder von betroffenen Personen so beschrieben wird, als wäre eine solche Gewebeschädigung die Ursache (Merskey und Bogduk 1994).

Die Patienten kommen häufig mit Beschwerden, die Sie als Schmerz beschreiben. Die zahnärztliche Schmerzanamnese sollte Lokalisation, Intensität, auslösende Faktoren, Beginn, zeitlichen Verlauf, Qualität und modifizierende Faktoren einbeziehen, um die Ursache des Schmerzes identifizieren zu können. Im Unterkiefer kann der Weisheitszahn Schmerzen bis in die Nähe des Ohres auslösen oder bis in den Bereich des Kehlkopfes (Weber 2000), was eine Folge von Karies, Parodontopathien, Entzündungsprozessen oder auch Traumata sein kann. Wenn keine alternativen Therapiemöglichkeiten gegeben sind, sollte man den Weisheitszahn entfernen, um die Schmerzursache zu beseitigen.

\section{- Nicht behandelbare Karies}

Karies ist die häufigste Erkrankung der Zahnhartsubstanz, die durch potentiell pathogene Mikroorganismen, niedermolekulare Kohlenhydrate und mit einer ausreichenden Einwirkzeit am Zahnschmelz entsteht (Hellwig et al. 1999). Durch eine optimale Mundhygiene und zahn- 
freundliche Ernährung kann man einer Karies grundsätzlich vorbeugen. Dennoch sind Weisheitszähne durch die schwer zugängliche Stellung im Zahnbogen besonders von Karies gefährdet, weil die Mundhygiene selbst für Erwachsene nicht einfach ist.

Das Ausmaß der entstehenden Karies bleibt nicht gering und eine Therapie ist oft prognostisch nicht mehr gerechtfertigt. Hier wird die Indikation zur Weisheitszahnentfernung wahrgenommen und der Zahn wird frühzeitig entfernt, um Entzündungen der Pulpa wie auch im Nachbargewebe zu vermeiden.

\section{- Parodontale Erkrankungen und nicht behandelbare periapikale Veränderungen}

Parodontitis marginalis ist eine entzündliche Erkrankung des Zahnbettes, die aufgrund bakterieller Stoffwechselprodukte hervorgerufen wird. Folgen dieser Krankheit sind Attachmentverlust, Knochenabbau und in fortgeschrittenen Stadien Zahnlockerung, Zahnverluste oder Abszesse. Die Therapie der Wahl wäre eine parodontale Behandlung. Da aber eine Parodontitis irreversibel und im Weisheitszahnbereich schwer zu therapieren ist, sollte diese Infektionsquelle durch eine Entfernung des Zahnes beseitigt werden.

Als periapikale Veränderung wird am häufigsten die apikale Parodontitis angesprochen. Die apikale Parodontitis ist ein entzündlicher Prozess an der Wurzelspitze des betroffenen Zahnes im Desmodont und im angrenzenden Knochen. Die Bakterien passieren durch den Wurzelkanal bis zur Wurzelspitze und lösen eine Entzündung aus, wobei zur Bekämpfung die Makrophagen des menschlichen Immunsystems Enzyme ausschütten, die gleichzeitig auch den Knochen auflösen. Eine Beseitigung dieser Bakterien wäre die Therapie der Wahl, welche in diesem Fall eine endodontische Behandlung oder auch eine Wurzelspitzenresektion wäre. Jedoch wird bei Weisheitszähnen aufgrund der anatomisch variierenden Wurzelkanalverhältnisse sowie häufigen Wurzelkanalkrümmungen eine Wurzelkanalbehandlung selten durch-geführt. 
Auch bei der Wurzelspitzenresektion sind diese Faktoren ein Problem. Zusätzlich erschwert im Unterkiefer die nahe Beziehung zum Nervus alveolaris inferior die Behandlung.

\section{- Resorption an benachbarten Zähnen}

Wurzelresorptionen an vitalen oder devitalen Zähnen beider Dentitionen sind die Folge dentoklastischer Zellaktivität, die reversibel oder irreversibel verlaufen können (Schroeder 1997).

Die physiologische Wurzelresorption wird in der Regel nur bei Milchzähnen beobachtet. Mit regressiven Veränderungen des Pulpa-gewebes wird die Regenerationsleistung verringert, und somit die Bildung von Tertiärdentin aufgehoben. Anstelle dieser Prozesse überwiegen Resorptionsvorgänge.

Die pathologische Resorption hingegen kann extern, intern oder auch als Druckresorption aufgrund anderer pathologischer Prozesse (z. B. Trauma, Tumor) oder abnorm durchbrechender Zähne verursacht werden (Gold und Hasselgren 1992).

Besonders horizontal und mesioangulär verlagerte dritte Molaren haben einen Einfluss auf die Wurzeln der Nachbarzähne (Azaz und Taicher 1982). In der Literatur wurde eine Resorption mit einer Inzidenz von 0\% bis $4,7 \%$ beschrieben (Von Wowern und Nielsen 1989; Nordenram et al. 1987).

\section{- Zähne im Bruchspalt, die die Frakturbehandlung erschweren}

8\% aller Unterkieferfrakturen im Kieferwinkel verlaufen durch eine Alveole (Schilli und Krekeler 1984). Besonders die retinierten Weisheitszähne bilden im Kieferwinkel eine Prädilektionsstelle für Frakturen. Bei teilretinierten Weisheitszähnen wie auch bei einer offenen Fraktur, die durch Gewalteinwirkung entstanden ist, kann es zu einer Bruchinfektion kommen, da eine Verbindung zwischen Mundhöhle und Knochen besteht. Hier ist eine Entfernung des Weisheitszahnes für eine infektionslose Frakturheilung indiziert. Auch nicht erhaltungswürdige Zähne wie z. B. 
Zähne mit Parodontitis apicalis, Wurzelfrakturen und großen kariösen Defekten sollten entfernt werden. Eine Ausnahme stellen impaktierte Zähne dar oder geschlossene Frakturen. Wenn eine optimale Reposition ohne Probleme möglich ist, können diese Weisheitszähne unter Antibiotikaschutz belassen werden.

\section{- Zähne, die zur Transplantation verwendet werden}

Beim vorzeitigen Verlust oder einer Nichtanlage des ersten Molaren kann anstelle dessen der Weisheitszahn transplantiert werden. Hierbei ist es prognostisch günstiger, wenn der Weisheitszahn noch im Zahnkeimzustand ist. Das geeignete Alter für eine Transplantation liegt zwischen dem 10. und 25. Lebensjahr und die Zahnwurzel sollte mindestens zu 2/3 oder besser $3 / 4$ ausgebildet sein, um eine hohe Erfolgsate zu erzielen (Schwenzer und Ehrenfeld 2009).

Des Weiteren gibt es die Indikation bei:

- Manifester Störung der dynamischen Okklusion durch Kippung oder Elongation.

- Zähnen, die bei der kieferorthopädischen und rekonstruktiven Chirurgie stören

\subsubsection{Prophylaktische Indikation}

Die prophylaktische Indikation kann sich aus der Abwägung der Risiken des Belassens gegenüber den Risiken der Zahnentfernung ergeben. Aus übergeordneten, der Lebensführung zuzuordnenden Gesichtspunkten, z. B. bei fehlender Verfügbarkeit medizinischer Versorgung etc., kann eine Entfernung sinnvoll sein.

Auch bei anderen Maßnahmen, die unter Narkose vorgenommen werden müssen, können Weisheitszahnentfernungen kombiniert werden, damit ein weiterer Narkoseeingriff nicht durchgeführt werden muss.

Bei geplanter prothetischer Versorgung, wenn ein sekundärer Durchbruch aufgrund der weiteren Atrophie des Alveolarkamms bzw. aufgrund der 
Druckbelastung durch herausnehmbaren Zahnersatz zu erwarten ist, kann nach Absprache mit dem Patienten eine prophylaktische Entfernung des Weisheitszahnes durchgeführt werden.

Auch im Gebiet der Kieferorthopädie kann zur Vereinfachung der kieferorthopädischen Zahnbewegungen und/oder zur Erleichterung der kieferorthopädischen Retention die Indikation gestellt werden (Wagner et al. 2009).

In der Literatur wurden prophylaktische Indikationen bei jugendlichen Patienten bis zu einem Alter von 24 Jahren vorgeschlagen, da der Weisheitszahn im Germzustand leichter zu entfernen ist und somit auch weniger Komplikationen aufweist (Chiapasco et al. 1995).

\subsubsection{Kontraindikation}

Als Kontraindikation der operativen Weisheitszahnentfernung sind akute Entzündungsereignisse wie Perikoronitis und Abszesse oder akute Schleimhauterkrankungen (Pilze, Herpes) zu erwähnen.

Aus allgemeinmedizinischer Sicht werden Patienten mit hämorrhagischen Diathesen, Antikoagulanzientherapie, immunsuppressiver Therapie, kardiovaskulären Erkrankungen, Schmerzmittelabusus und Zustand nach einer Strahlentherapie im Mund-Kiefer-Gesichtsbereich als kontraindiziert angesehen, wobei nach Absprache mit dem Internisten eine Operation auch möglich ist.

\subsection{Operationstechnik}

Bei Zähnen, deren Extraktion aufgrund ihrer Retention und Verlagerungsart nicht im Sinne einer Extraktion möglich sind, müssen diese operativ entfernt werden. Diese Entfernung kann grundsätzlich als Osteotomie erfolgen (Klammt 2000). In der Literatur wurden aufgrund der zahlreichen Komplikationen mehrere Methoden der operativen Weisheitszahnentfernung beschrieben. 


\subsubsection{Weichgewebspräparation}

Der erste Schritt einer Osteotomie ist die Darstellung des Operationsfeldes, die durch eine Inzision erfolgt. Die Inzision wird je nach Situation unterschiedlich gewählt, wobei verschiedene Anforderungen erfüllt werden sollten. Wichtig ist hierbei, dass eine ausreichende Übersicht, Schnittführung mit Abstand zum Knochendefekt, Erweiterungsfähigkeit, spannungsfreie Verschlussmöglichkeiten und keine Gefährdung der Lappengefäße gewährleistet werden sollte.

$\mathrm{Da}$ in der Literatur Zusammenhänge zwischen der Dimension der Wundfläche und Wundinfektionsrate oder auch der Knochenresorption beschrieben wurden (Goetzke und Klammt 1991), konnten demnach verschiedene Inzisionstechniken entstehen.

\subsubsection{Winkelförmige Inzision}

Die als Standard-Inzision durchgeführte winkelförmige Inzision dient zur kompletten Übersicht des Operationsfeldes.

Als erstes wird ein Schnitt, mittig oder vestibulär vom distalen Kronenanteil des zweiten Molaren, durchgeführt. Aufgrund der möglichen Traumatisierungen des oberflächig verlaufenden Nervus lingualis erfolgt eine relativ spitzwinklige Schnittführung nach vestibulär dorsal, wobei sie stets auf der Knochenunterlage Kontakt aufweist.

Als zweites wird die Schnittührung nach mesial durchgeführt, die am marginalen Rand entlang des zweiten Molaren und ins Vestibulum hin entlastet wird (Wagner et al. 2009). Hierbei sollte die Inzision nicht über die Umschlagsfalte reichen, da die Gefahr der Verletzung der Fazialisgefäße besteht. Neben dieser marginalen Schnittführung gibt es auch die Möglichkeit, den Schnitt paramarginal zu führen, um die marginale Gingiva zu schonen (Suarez-Cunqueiro et al. 2003; Kirtiloglu et al. 2007).

Nach der Inzision wird ein Mukoperiostlappen gebildet, indem man supperiostal die Schleimhaut samt Periost vom Knochen mit einem 
Raspatorium abscharbt.

Der Vorteil dieses Lappens ist, dass diese Inzision alle Anforderungen erfüllt, und bezüglich der Technik für unerfahrene Operateure einfach in der Durchführung ist.

Als Nachteil wird die Knochenresorption durch den gebildeten Mukoperiostlappen belegt (Brägger et al. 1988; Fickl et al. 2011), die durch die stimulierte Osteoklastenaktivität zustande kommt. Zusätzlich ist auch das Infektionsrisiko durch eine großflächige Wunde erhöht.

\subsubsection{Spaltlappen-Inzision (Partial-Thickness Flap)}

Die Durchführung der Spaltlappen-Inzision wurde bereits in den 60er Jahren in der Literatur erwähnt (Pfeiffer 1965). Dennoch wurde die erste richtige Klassifizierung in Mukoperiost- (Full-Thickness Flap) und Spaltlappen (Partial-Thickness Flap) erst später richtig definiert.

Der in der Oralchirurgie als "Standard-Verfahren“ durchgeführte Mukoperiostlappen ist, wie der Name schon sagt, ein Lappen, der aus allen Schichten der Schleimhaut, einschließlich des Periostes besteht. Der Spaltlappen hingegen besteht nur aus der epithelialen Schicht und der zugrunde liegenden Bindegewebsschicht, ohne das Periost. Diese Methode der Lappenbildung wurde überwiegend in der Parodontologie verwendet, um die Knochenresorption durch Abpräparieren des Periostes zu reduzieren. $\mathrm{Da}$ in der Oralchirurgie das gleiche Problem der Knochenresorption (Brägger et al. 1988; Fickl et al. 2011) bestand, wurde die Etablierung dieser Technik versucht.

In der Literatur konnten auch im Gebiet der Oralchirurgie, wie z. B. in implantologischen oder augmentierenden Bereichen, reduzierte Knochenresorptionen im Zusammenhang mit der Nutzung von Spaltlappen nachgewiesen werden (Jensen et al. 2009; Fickl et al. 2011). Die Technik dieser Lappenbildung ist anspruchsvoller als ein Mukoperiostlappen und ist somit für Operateure mit geringer Erfahrung schwieriger zu praktizieren. Aufgrund dessen ist derzeit diese Inzision im Gebiet der Weisheitszahnosteotomie nicht weit verbreitet. 


\subsubsection{Komma-Inzision}

Die Komma-Inzision ist, wie der Name bereits besagt, eine Schnittführung, die dem Satzzeichen „Komma“ ähnelt.

Im Jahre 2002 wurde diese Inzision durch Nageshwar definiert und vorgestellt. Die Schnittführung bei dieser Inzision beginnt mit einer Dehnung der vestibulären Schleimhaut nach kaudal durch den Zeigefinger oder Daumen, was eine Dehnung des Bukkinatormuskels erzeugt. Ausgehend von einem Punkt an der vestibulären Schleimhaut erfolgt danach ein Schnitt distal des zweiten Molaren, aufsteigend bis zum distobukkalen marginalen Rand und folgt dem Zahn bis zur lingualen Seite.

Das resultierende Operationsfeld erlaubt dem Operateur fast alle Osteotomietechniken wie z. B. konventionelle Osteotomie oder die linguale Split-Technik. Durch eine kleine Modifikation können nicht nur impaktierte Zähne, sondern auch teilretinierte Zähne freigelegt werden.

Vorteil dieser Inzision ist das minimal-invasiv gehaltene Operationsfeld, wobei eine Lappenbildung nicht mehr nötig ist. Die Wunden liegen nicht auf den knöchernen Defekten, Muskeln oder Sehnen. Nach der Entfernung des Zahnes ist auch die Replazierung der Schleimhaut einfach zu gestalten. Auch von postoperativen Ergebnissen konnte man in dieser Studie feststellen, dass in Hinsicht auf Schmerz, Schwellung, Trismus und parodontales Attachment die Auswertung besser war, als bei der konventionellen Inzision (Nageshwar 2002).

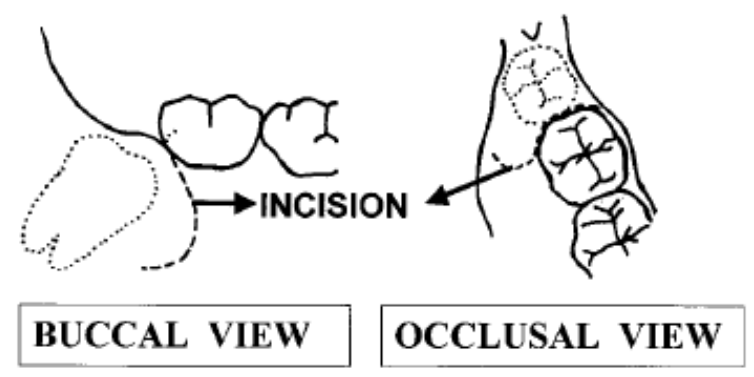

Abb.1.4: Bildliche Darstellung der Komma-Inzision (Nageshwar 2002, S.1507) 


\subsubsection{Flapless-Inzision}

Die Flapless-Inzision wurde bei teilretinierten Weisheitszähnen in der Vergleichsstudie von Kim et al. im Jahre 2011 vorgestellt.

In dieser Studie wurden die teilretinierten Weisheitszähne bei Patienten mit oder ohne Lappenbildung entfernt. Der chirurgische Eingriff erfolgte ansonsten mittels eingesetzter Hartmetall-Bohrer, die den Zahn in zwei Fragmente zerteilte, auf gleiche Weise.

Diese Studie belegt, dass die Entfernung eines teilretinierten Weisheitszahnes ohne Lappenbildung in Hinsicht auf Schmerz, Schwellung, Sondierungstiefe von Patienten besser empfunden wird, als eine Entfernung mit Lappenbildung.

Analog zu der Studie von Choi und Engelke (2009), die die FlaplessTechnik in der Implantologie belegt haben, waren die gleichen Vorteile zu beobachten: eine Reduktion des Weichgewebstraumas, geringere Narbenbildung, Erhalt der Vaskularisation des Knochengewebes und strukturelle Vorteile der marginalen Gingiva des Nachbarzahnes.

Dennoch kann nur Flapless operiert werden, wenn die Voraussetzung besteht, dass eine ausreichende Übersicht des Operationsfeldes gewährleistet ist. Dies bedeutet, der Weisheitszahn sollte entweder vollständig oder partiell in der Mundhöhle durchgebrochen sein.

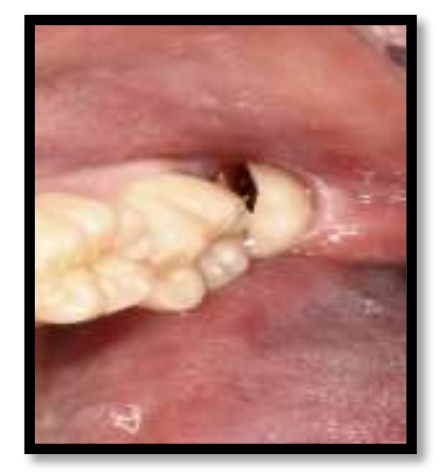

Abb.1.5: Bildliche Darstellung der Flapless-Odontotomie: Trennung des Weisheitszahnes ohne vorangegangene Inzision (Kim et al. 2011, S.968) 


\subsubsection{Hartgewebspräparation}

\subsubsection{Vestibuläre Osteotomie}

Die vestibuläre Ostetomie ist uns bekannt als die „konventionelle Methode“. Nach der Darstellung des Alveolarknochens wird die Schleimhaut mithilfe von Wundhaken abgehalten. Vestibulär und distal des retinierten Zahnes wird der Knochen bis auf den maximalen Kronenumfang mittels Fräsen und Rosenbohrer unter Wasserkühlung abgetragen. Wenn es unvermeidlich ist, an der lingualen Fläche des Alveolarkammes Knochen abzutragen, wird zum Schutz des Nervus lingualis ein Raspatorium subperiostal eingeführt.

Die Nutzung dieser Retraktionshilfsmittel wird in der Literatur oftmals diskutiert. Zum einen wird belegt, dass die Instrumente, die für den eingentlichen Schutz dienen sollten, eine Ursache der Nervschädigung darstellen (Blackburn und Bramley 1989; Robinson und Smith 1996). Andererseits gibt es Studien, die das Gegenteil behaupten (Appiah-Anane und Appiah-Anane 1997; Pogrel und Goldman 2004).

Ein großer Teil der teilretinierten Zähne sollte nach dem vestibulären Knochenabtrag entfernbar sein. Je nach Verlagerungsart wird weiterer Knochen osteotomiert, sodass der Zahn ohne größere Kraftanwendung mit dem Hebel luxiert werden kann. Bei morphologisch schwierigen Zähnen wäre sonst eine Odontosektion empfehlenswert, um weitgehend den Knochen in der Umgebung wie auch den Alveolarnerven zu schonen.

Diese Technik kann allgemein auch von nicht erfahrenen Zahnärzten durchgeführt werden, da die Methode relativ einfach ist. Auch die gute Übersicht der Alveole ist bezüglich verbliebener Wurzelreste von Vorteil. Jedoch ist die Menge des Knochenverlustes bei komplizierten Zähnen erheblich, sodass sogar postoperative Komplikationen wie z. B. Nervenschädigungen, Kieferfrakturen oder auch Infektionen auftreten können (Dunstan und Sugar 1997; Barbosa-Rebellato et al. 2011). 


\subsubsection{Linguale Split-Technik}

Die linguale Split-Technik wurde im Jahre 1956 von Ward publiziert und später durch Davis et al. (1983) modifiziert vorgestellt. Die erste Idee dieser Prozedur war, nach Bildung eines Weichteillappens die linguale Knochenplatte zu entfernen, und so den Zugang zum Weisheitszahn zu erzielen. Anders als bei der konventionellen Osteotomie wurden hierbei weder Fräse noch Rosenbohrer verwendet, sondern ein Osteotom.

Das Problem bei dieser Operation war, dass es zu exzessiven Blutungen kam, und dies die Operation erschwerte.

Die spätere Modifikation löste das Problem, indem die linguale Knochenplatte nicht mehr komplett vom Periost getrennt wurde. Begleitend erfolgte auch eine Modifikation der eigentlichen Osteotomie. Die Osteotomie der lingualen Knochenplatte wurde nicht in einem ganzen Stück, sondern in mehreren Fragmenten geteilt durchgeführt, um gezielter mit dem Osteotom den Weisheitszahn freizulegen.

Mit dieser Technik konnten alle Angulationstypen routiniert entfernt werden (Davis et al. 1983), was allerdings eine Einweisung in die verschiedenen Vorgehensweisen voraussetzte. Zusätzlich sollte auch eine Einarbeitung mit dem Osteotom erfolgen, damit eine Fehlanwendung und dadurch resultierende Kieferfrakturen nicht entstehen.

Nach routinierter Anwendung kann diese Technik zeitsparender als die konventionelle Technik sein (Hindy et al. 1995), was von den Patienten aufgrund postoperativer Schmerzen, die stärker als bei der konventionellen Technik bewertet werden, nicht als ein erheblicher Vorteil wahrgenommen wird (Praveen et al. 2007).

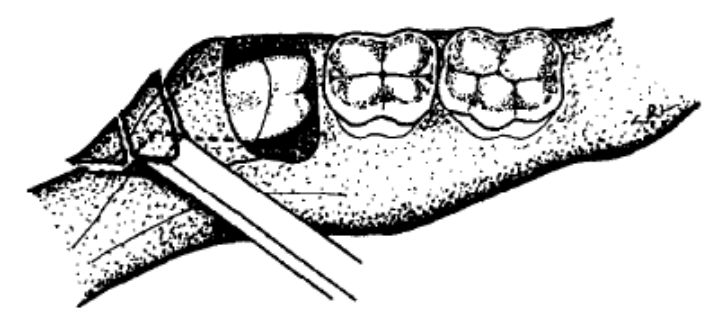

Abb.1.6: Bildliche Darstellung der lingualen Split-Technik (Davis et al.1983, S.3) 


\subsubsection{Koronektomie}

Die Koronektomie, oder auch partielle Weisheitszahnentfernung genannt, wurde zur Protektion des Nervus alveolaris inferior entwickelt. Hierbei erfolgte eine gezielte Entfernung des Kronenanteils vom Weisheitszahn, wobei der Wurzelanteil im Kochen (in situ) belassen wurde. Mit der Zeit sollte sich der Wurzelanteil allmählich in krestaler Richtung bewegen und somit einer Nervenschädigung vorbeugen.

Ziel dieser Technik war, eine mögliche Läsion, die bei der konventionellen Osteotomie aufgrund einer engen Beziehung zwischen Nervus alveolaris inferior und dem Zahn entstehen kann, zu vermeiden. Auch die Infektion bei einer Perikoronitis sollte durch die Entfernung der Krone eliminiert werden.

Die erste Publikation zu dieser Technik wurde im Jahre 1984 von Ecuyer und Debien veröffentlicht, gefolgt von weiteren Studien wie z. B. von Knutsson et al. (1989). Dabei gab es Berichte über Komplikationsraten von 27\%. Mit der Zeit wurden weitere Verbesserungen der Trennmethode und Ausschlusskriterien vorgeschlagen.

In der Studie von Pogrel et al. (2004) erfogte die Entfernung der Krone mit einer $45^{\circ}$ igen Odontosektion und einem Abtrag der Zahnsubstanz bis auf $3 \mathrm{~mm}$ unter dem Knochenniveau. Um eine hohe Komplikationsrate zu vermeiden, wurden strenge Ausschlusskriterien aufgestellt. Horizontal angulierte Zähne, akut infizierte Zähne und mobile Zähne wurden ausgeschlossen.

Die praktische Durchführung bei dieser Technik ist im Vergleich zu anderen Techniken nicht anspuchsvoller. Aber die Komplikationsrate wie auch die strengen Ausschlusskriterien werden hierbei als Nachteil gesehen. 


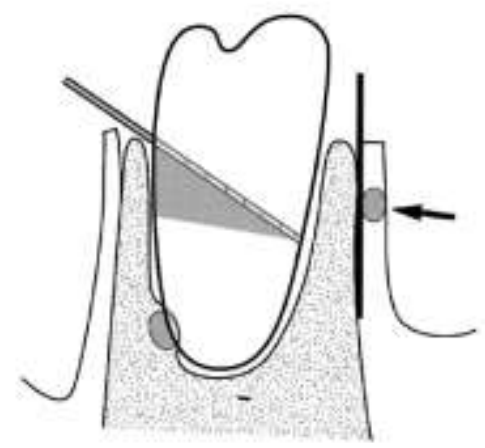

Abb.1.7: Bildliche Darstellung der Koronektomie (Pogrel et al. 2004, S.1448)

\subsubsection{Perikoronale Ostektomie-Technik}

Ausgehend von der Koronektomie wurde 2011 von Tolstunov et al. die Technik „Perikoronale Ostektomie“ vorgestellt.

Das Prinzip, den Weisheitszahn leicht eruptieren zu lassen, um eine Distanz zum Nervus alveolaris inferior zu erzielen, ist gleichgeblieben. Die Raumschaffung im Knochen, den der Zahn benötigt, um zu eruptieren, wird anders als bei der Koronektomie durch Abtragen des perikoronalen Knochens geschaffen. In der zweiten Operationsphase, die nach ca. sechs bis acht Wochen erfolgt, wird der leicht eruptierte Weisheitszahn komplett entfernt.

Vorteil dieser Technik ist, dass weniger Nervenläsionen entstehen und die Heilung der Alveole einer normalen Extraktionsalveole gleicht. Die Kontraindikation bei distoangulären sowie bei horizontal angulierten Weisheitszähnen bleibt gleich.

Als Nachteil dieser Prozedur wird die zweiphasige Operation genannt, welche für die Patienten eine doppelte Belastung bedeutet.

\subsubsection{Wundversorgung und Nachbehandlung}

Nach Exposition des dritten Molaren wird abschließend kürettiert, um das Weichgewebe, wie z. B. Zahnsäckchen oder Zystengewebe, zu entfernen. Anschließend wird eine Glättung der Knochenkanten wie auch eine Spülung mit physiologischer Kochsalzlösung durchgeführt. Je nach Größe 
der Weichteilwunde und Infektionsgefahr kann die Wunde offen, halboffen oder geschlossen behandelt werden.

Die offene Wundversorgung wird bei Weisheitszahnentfernungen im Unterkiefer oft bevorzugt, um Infektionsgefahren zu vermeiden. Es wird eine Tamponade in die Wunde eingelegt, wobei die Weichteilwunde mit Nähten adaptiert wird. Bei größeren Knochenwunden kann zur Stabilisierung des Blutkoagulums oder auch des Knochendefektes ein Kollagenkegel oder Kollagenvlies eingelegt werden. Zwar ergibt sich eine verzögerte Wundheilung und die Nachsorge ist aufgrund ständiger Drainage aufwendiger, allerdings wird das postoperative Komplikationsrisiko damit gesenkt.

Die halboffene Wundversorgung kann bei primär infizierten Wunden oder als Alternative zur offenen Wundversorgung erfolgen, um die Nachsorgeintervalle zu verringern. Hierbei wird die Wunde verschlossen und nur zur vestibulären Seite eine Drainage durch einen Jodoformstreifen oder eine adaptierte Gummilasche zur Entlastung eingelegt. Nach ca. drei Tagen kann die Drainage entfernt werden.

Eine geschlossene Wundversorgung ist Voraussetzung einer primären Wundheilung und sichert gleichzeitig das Blutkoagulum. Bei größeren Knochenwunden, bei denen Defektfüllungen mit Knochenersatzmaterial oder regenerativen Membranen durchgeführt werden, ist ein Nahtverschluss obligatorisch. Für die Patienten ist diese Art der Wundversorgung angenehm und die Nachsorge ist bei gutem Heilungsverlauf nicht oft erforderlich.

Außerdem sollten die Patienten darüber aufgeklärt werden am Operationstag weiche Kost zu bevorzugen und Milchprodukte zu vermeiden. Ebenfalls sollte er bei starken Nachblutungen sowie Beschwerden sich melden. Der behandelnde Arzt sollte bei längeren und schwierigeren Eingriffen Antibiotika sowie Analgetika verschreiben 
Die nächste postoperative Kontrolle wird in der Regel nach 7-14 Tagen vereinbart. An diesem Termin werden die Fäden entfernt und die Wundverhältnisse überprüft.

\subsection{Literaturübersicht zur Auswertung der Knochendefektsituation}

Die unterschiedlichen Verfahren zur Analyse der knöchernen Defektsituation wurden schon bei vielen anderen wissenschaftlichen Arbeiten im Gebiet der Oralchirurgie, Implantologie und Parodontologie erwähnt und durchgeführt. Diese Verfahren sollen helfen eine räumliche Darstellung und Vermessung der Kiefer bzw. der Alveolen zu bekommen, um einerseits eine Operationsmethode positiv/negativ zu beurteilen und anderseits ein Ergebnis zur Fragestellung des Knochenverlustes zu erhalten.

\subsubsection{Modellvermessung}

Die Gipsmodelle, die in der zahnärztlichen Praxis alltäglich begutachtet werden, dienen für verschiedene Zwecke. Einerseits wird ein Ausgangszustand festgehalten, in dem Situationsmodelle hergestellt werden, um damit die Planung von Zahnersatz durchzuführen. Anderseits erfolgt die Herstellung von Zahnersatz auf angefertigten Modellen. Die Gipsmodelle werden auch im Gebiet der restaurativen Zahnmedizin, Kieferorthopädie und kraniomandibulären Dysfunktionen verwendet, um z. B. die Kieferverhältnisse zueinander zu analysieren.

Das Modell hat den Vorteil, bezahnte sowie auch unbezahnte Stellen des Kiefers detailgetreu wiederzugeben, damit die verschiedenen Situationen räumlich und bildlich dargestellt werden können. Diesbezüglich kann ein Modell auch zum prä- und postoperativen Vergleich, Ausmaß einer Extraktionswunde oder Knochendicke angewendet werden (Pietrokovski und Massler 1967). Allerdings ist die Befunderhebung bei dieser Methode auf koronale Zahnabschnitte beschränkt, sodass eine Aussage zur Beschaffenheit des Knochens oder der Zahnwurzel nicht erfolgen kann. 


\subsubsection{Röntgenologische Vermessung}

Die Befundung von Röntgenbildern dienen im zahnärztlichen Alltag sowohl der Diagnostik von pathologischen Prozessen als auch der Begutachtung von Zähnen, Knochen, Kiefergelenken sowie Zahnersatz und Restaurationen. Durch die Entwicklung der digitalen Geräte werden heutzutage immer mehr röntgenologische Aufnahmen zur Diagnostik und zur Operationsplanung verwendet, da durch genaue dreidimensionale Angaben und Vermessungen mögliche intraoperative Komplikationen zu vermeiden sind (Heurich et al. 2002).

Je nach Indikation werden durch verschiedene Verfahren unterschiedliche Röntgenbilder oder Dateien hergestellt, die zur Hilfestellung der Befundung dienen sollen.

Bei kleineren Bereichen des Kieferabschnittes können Einzelzahnfilme oder Bissflügelaufnahmen angefertigt werden, die zur Befundung von apikalen Herden oder einer Karies dienen (Pasler 1995). Eine komplette Übersicht der Zähne, Kieferknochen und Kiefergelenke kann durch eine Panoramaschichtaufnahme erfolgen. Wenn eine zweidimensionale Bildgebung nicht ausreichend ist, können zwei Verfahren kombiniert in zwei verschiedenen Ebenen aufgenommen werden, um z. B. die Lokalisation von pathologischen Prozessen oder die Lage von verlagerten Zähnen zu bestimmen (Pasler 1995). Im Gebiet der Oralchirurgie werden oft die digitalen Röntgengeräte verwendet, um genaue dreidimensionale Lokalisationen von Nachbarstrukturen zu bekommen. Auch in der Forschung werden oft im Rahmen einer Verlaufskontrolle röntgenologische Aufnahmen verwendet, um Ausmaß der bestehenden oder entstandenen Defekte etc. auszuwerten.

In der Literatur wurden, als eine mögliche Methode, prä- und postoperative Röntgenbilder verwendet, um die Knochendefektsituation, z. B. nach Entfernung der Weisheitszähne, zu vermessen. Ältere Studien zeigen eine 
Vermessung der Röntgenbilder anhand transparenter Lineale (Kugelberg et al. 1985). Mittlerweile können diese Röntgenbilder in Kombination mit einer Mess-Software digitalisiert aufgenommen, eingescannt und ausgemessen werden. Hierbei dienen Schmelz-Zement-Grenze des Nachbarzahnes und Alveolarkammoberfläche als Referenzstrukturen der Messung (Krausz et al. 2005; Andrade Munhoz et al. 2011).

\subsubsection{Intraorale Vermessung}

In vielen Studien wurden Parameter, die zur Bewertung der Knochendefektsituation beitragen konnten, durch intraorale Vermessungen ausgewertet (Caplanis et al. 2005). Mit Referenzhilfen wie z. B. einem Titan-Pin oder individuell angepassten Acryl-Schienen konnten die Knochendefekte bestimmt werden (lasella et al. 2003; Lekovic et al. 1997). Dazu dienten skalierte Hilfsmittel wie digitale Schiebelehren oder modifizierte Parodontalsonden. 


\subsection{Zielsetzung}

Ziel der vorliegenden Arbeit ist, eine neue Methode der operativen Entfernung eines Weisheitszahnes vorzustellen, welcher über einen okklusalen Zugang mikrochirurgisch entfernt wird.

Hierbei wird die Nutzung der visuellen Hilfsmittel je nach Verlagerungsart der Zähne wie auch der Operationsphasen untersucht. Die Operationsdauer sowie auch die Komplikationsrate und Knochendefekte werden ebenfalls ermittelt, um folgende Fragestellungen zu beantworten:

1. Können mithilfe von Mikroskop und Endoskop alle Strukturen bei der Zahnentfernung dargestellt werden?

2. Welche Vor- und Nachteile ergeben sich für die Anwendung von Endoskop und Mikroskop?

3. Kann eine Routine in der Anwendung der visuellen Hilfsmittel bei verschiedenen Angulationstypen definiert werden?

4. Wie hoch ist die Operationszeit?

5. Kann ein Zusammenhang zwischen den Angulationstypen und der Operationsdauer ermittelt werden?

6. Wie hoch ist der postoperative Knochenverlust?

7. Besteht ein Zusammenhang zwischen den Angulationstypen und dem Knochenverlust?

8. Wie hoch ist die intra- und postoperative Komplikationsrate? 


\section{Material und Methode}

\subsection{Untersuchungsgut}

Das Patientenkollektiv dieser Studie setzt sich aus 40 Patienten der Universitätsklinik Göttingen zusammen. Es wurden 19 Männer und 21 Frauen ohne bekannte Allgemeinerkrankungen im Alter von 15 bis 55 Jahren, im Zeitraum von April 2010 bis Juni 2011 untersucht und mit der minimal-invasiven „okklusalen Expositionstechnik“ operiert.

Es wurden 50 Zähne, 26 auf der linken Seite und 24 auf der rechten Seite des Unterkiefers, operativ entfernt. Die Einschlusskriterien für die Studie erfüllen Patienten, bei denen eine Indikation zur uni- oder bilateralen Unterkieferweisheitszahnentfernung bestand und die bereit waren, an der Studie teilzunehmen. Ausgeschlossen wurden unkooperative Patienten wie auch Patienten, die zur generellen Gruppe der Nichteinwilligungsfähigen gehörten.

Zu Beginn wurde von allen Patienten die Anamnese erhoben, worauf hin die intraorale Befundaufnahme sowie die Röntgenuntersuchung erfolgte. Bei einem Verdacht auf eine enge Beziehung zum Nervus alveolaris inferior, die man anhand der Panoramaschichtaufnahme feststellen konnte, wurde zusätzlich noch eine Aufnahme mittels digitaler Volumen-Tomographie (DVT) angefertigt. Die Verlagerung der Zähne wurde anhand der Klassifikation nach Winter (1926) aufgeteilt.

Die Patienten wurden ausführlich über die Einzelheiten der Vorgehensweise sowie eine alternative konventionelle Vorgehensweise informiert, aufgeklärt und auf mögliche Komplikationen hingewiesen. Sie gaben ihr schriftliches Einverständnis für die Anwendung der okklusalen Expositionstechnik. 


\subsection{Instrumentarium}

\section{Endoskop}

Bei der folgenden Studie erfolgten sämtliche Eingriffe mit einem Stützimmersionsendoskop, welches unterstützend für eine Übersicht des Operationsfeldes wie auch zur Beurteilung der Morphologie und der knöchernen Defektsituation der Extraktionsalveole ohne Lappenbildung gut geeignet ist (Juodzbalys et al. 2008).

Das Endoskop ist mit einer Storz-Hopkins-Optik (Durchmesser 2,7 mm, 30 Vorausblick, Fa. Storz, Tuttlingen) und mit einem Stütz-Spülschaft bestückt (Abb.2.1). Die gesamte Operation konnte mithilfe eines Videorekorders über die Endoskopkamera aufgezeichnet werden.

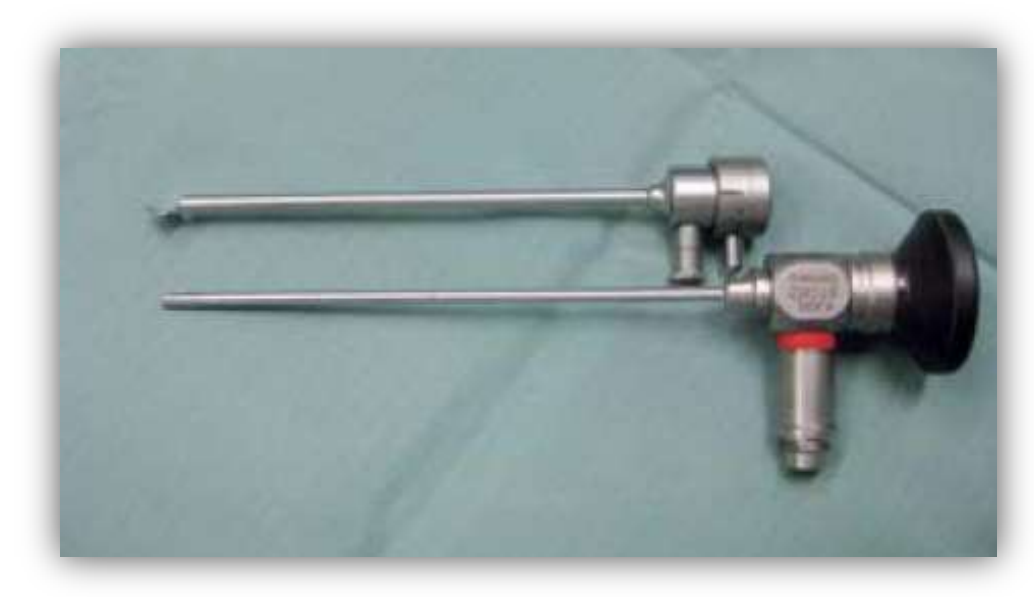

(a)

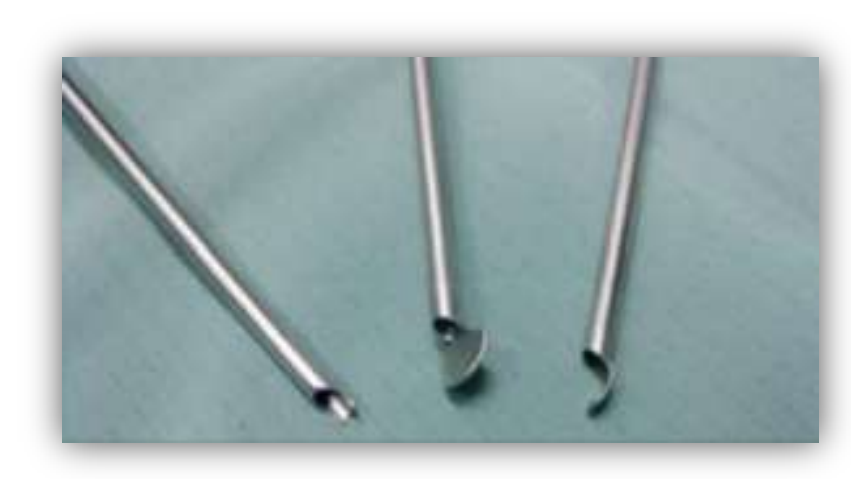

(b)

Abb.2.1: a) Storz-Hopkins-Optik mit Stütz-Spülschaft

b) Verschiedene Stütz-Spülschäfte zur Aufnahme der Optik

(Engelke et al. 2011, S.416) 


\section{Mikroskop}

Die Anwendung des Operationsmikroskops in der Oralchirurgie sowie in anderen Bereichen wurde bereits ausführlich beschrieben (GarciaCalderon et al. 2007).

In dieser Studie wurde analog zum Endoskop eine visuelle Unterstützung durch ein Operationsmikroskop (Zeiss OPMI) gewährleistet (Abb.2.2). Durch die interne Lichtquelle und die bis zu 40-fache Vergrößerung der Optik wird die Übersicht des Operationsfeldes gesichert. Hierbei konnte eine Aufzeichnung durch eine Software digitalisiert erfolgen.

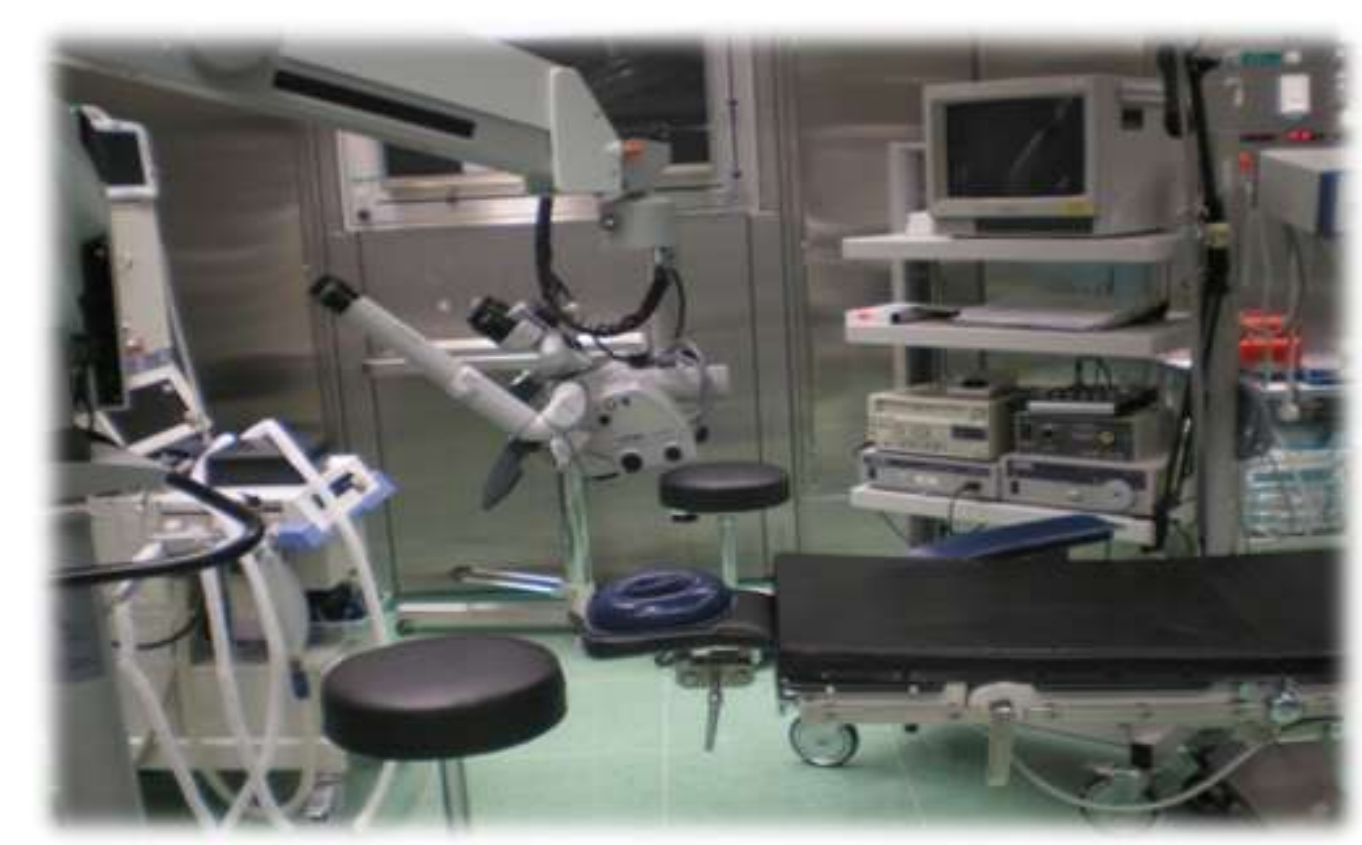

Abb.2.2: Darstellung des Mikroskops im Operationssaal

\section{Operationsbesteck}

Für die Operation wurde das Osteotomiebesteck verwendet. Außerdem erfolgte eine Anwendung des Handstücks mit Lindemannfräse, Rosenbohrer und kugelförmigen Diamanten zur Odontosektion.

Neben der Anwendung von Bein- und Flohr-Hebeln wurden auch Heidbrink-Elevatoren (Hu-Friedy) und Mikroelevatoren zur Entfernung von Zahn-fragmenten genutzt. In der Gruppe von Heidbrink-Elevatoren wurden besonders die grazilen Wurzelspitzenheber zur Entfernung von Wurzeln in 
schwer zugänglichen Bereichen verwendet.

\subsection{Chirurgisches Vorgehen}

\section{Die Anästhesie}

Alle chirurgischen Eingriffe wurden mit Ultracain-DS (Articain 2\% mit 1:1000000 Adrenalinzusatz) unter Leitungsanästhesie im Bereich des Nervus alveolaris inferior und Nervus buccalis sowie mit einer Lokalanästhesie im Bereich des zweiten und dritten Molaren durchgeführt. Die Durchführung erfolgte am liegenden Patienten mit einer Injektionsmenge von $1,5 \mathrm{ml}$ für die Leitungsanästhesie und $1,0 \mathrm{ml}$ für die Lokalanästhesie.

Nach einer ausreichenden Einwirkzeit des Anästhetikums erfolgte der mikrochirurgische Eingriff. Der Operateur sitzt hierbei auf 12-Uhr-Position und die Assistenz auf der linken Seite des Patienten, wobei der Kopf vom Patienten leicht rekliniert gelagert sein sollte.

\section{Die Inzision}

Es erfolgte als erstes die Exposition des Operationsfeldes unter Sicht des Operationsmikroskops oder mit der Lupenbrille.

Der Zugang erfolgte im Sinne einer selektiv okklusalen Aufklappung unter Bildung eines Mini-Flaps ohne laterale Lappenbildung (Mini-Flap-Inzision). Der Mukoperiostlappen hat die Aufgabe das Operationsfeld übersichtlich zu gestalten, was bei der mikrochirurgischen Technik durch Endoskop und Mikroskop gewährleistet wird und somit nicht erforderlich ist. Bei vollständig retinierten oder auch bei impaktierten Zähnen würde aufgrund dessen eine relativ minimale Inzision von ca. $1,5 \mathrm{~cm}$ ausreichen. Beginnend mit einem Schnitt distobukkal am aufsteigenden Ast des Kiefers wurde dies bis zum Sulkus des zweiten Molaren im lingualen Drittel des Kieferkammes geschwenkt. Die Inzision folgte dem Sulkus entlang und endete mit einer vestibulären Entlastung (Abb.2.3). 


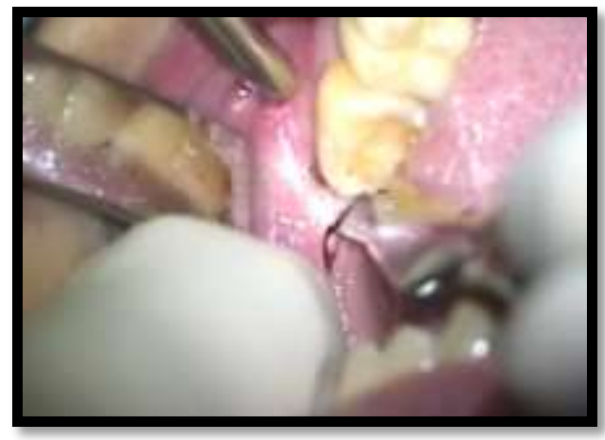

(a)

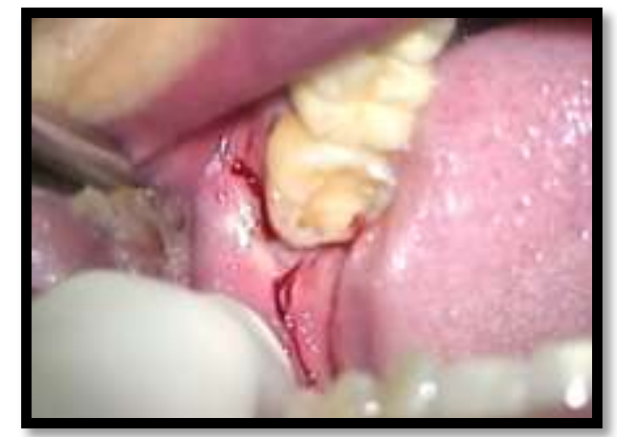

(b)

Abb.2.3: a und b: Inzision ohne Bildung eines großflächigen Mukoperiostlappens (Mini-Flap-Inzision) beim vollretinierten Weisheitszahn

Es folgte nach dem Ablösen des okklusalen Weichgewebes die Darstellung der lingualen und bukkalen Begrenzung der Kieferkammoberfläche. Ein Wundhaken (modifizierter Langenbeck-Haken) wurde auf der bukkalen Seite eingesetzt, ohne dass eine kaudal gerichtete Ablösung des Mukoperiostlappens erfolgte. Auf einen lingualen Wundhaken wurde in der Regel verzichtet (Abb.2.4).

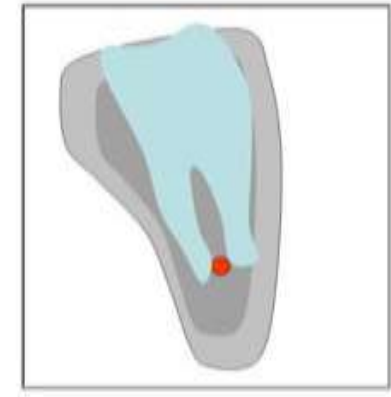

(a)

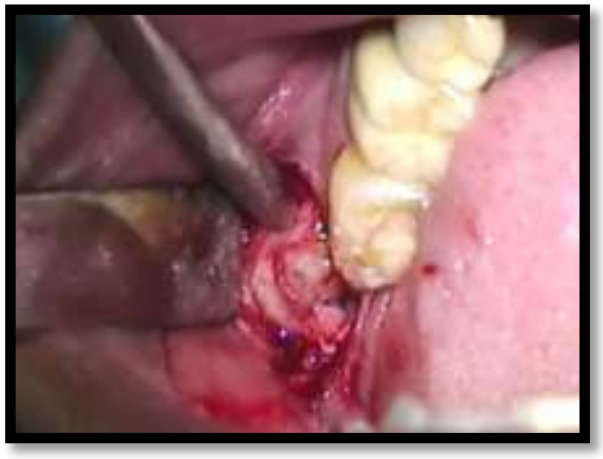

(b)

Abb.2.4: Exposition der Knochenoberfläche

a) Schematische Darstellung

b) Mikroskopische Aufnahme

Nach Darstellung der Knochenoberfläche in der Region des dritten Molaren konnte im distalen Bereich des Operationsfeldes das Stützendoskop (Karl Storz, Tuttlingen, Deutschland) eingesetzt werden. Als Abstandshalter zur Knochenoberfläche diente der Spatel des Stützendoskops, der gleichzeitig auch das Weichgewebe separierte. Um eine gute Übersicht zu 
gewährleisten, wurde je nach Lage des Zahnes mit dem Mikroskop und/oder mit dem Stützendoskop weitergearbeitet (Abb.2.5).

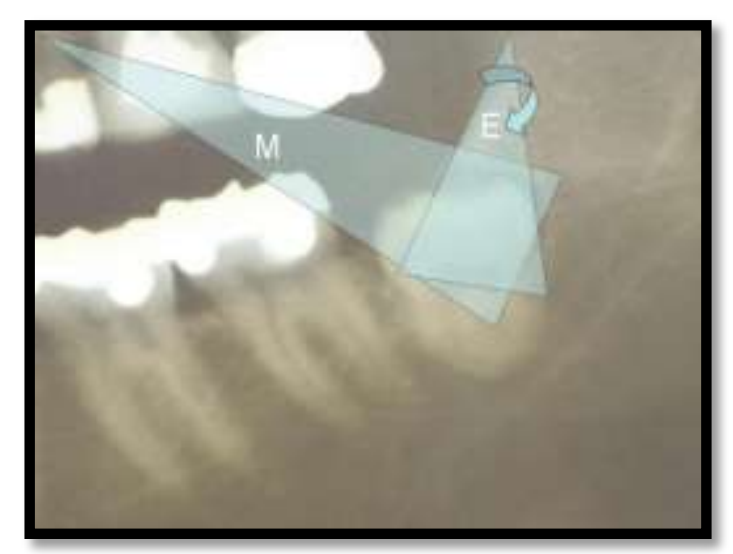

Abb.2.5: Mikrochirurgische Visualisierung des Operationsfeldes

M: Einblick mit Mikroskop oder Lupenbrille von lateral anterior

E: Einblick mit Stützendoskop von posterior superior

(Engelke et al. 2011, S.417)

\section{Die Osteotomie}

Nach Exposition der Knochenoberfläche wurde die okklusale Exposition der Krone mittels Osteotomie durchgeführt. Hierbei wurde ein Rosenbohrer unter Wasserkühlung angewendet, bis der nach okklusal zeigende Teil der Krone exponiert war. Das bedeutet, dass nur so viel Knochen osteotomiert wurde, bis man den Zahn an der Oberfläche erkennen konnte, unabhängig von der Position des Zahnes im Kiefer (Abb.2.6). 


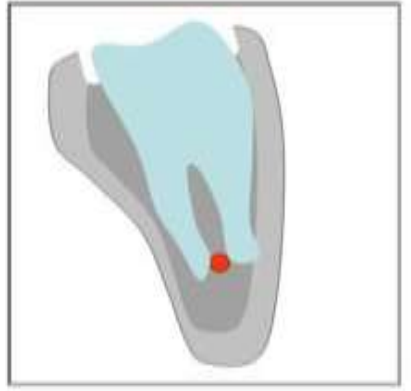

(a)

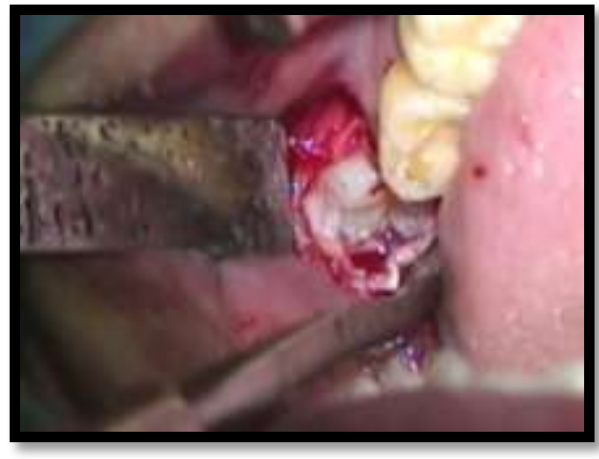

(b)

Abb.2.6: Okklusale Exposition

a) Schematische Darstellung

b) Mikroskopische Aufnahme

\section{Phase 1 - Raumschaffende Trepanation}

Als nächstes wurde die transversale raumschaffende Trepanation durchgeführt. Dabei wurde das bukkale und mittlere Drittel der Krone mit einer Lindemannfräse transversal eröffnet und mithilfe von Rosenbohrern und diamantierten Fräsen erweitert (Abb.2.7).

Diese raumschaffende Präparation wurde, je tiefer und näher die benachbarten Knochenstrukturen wie auch Gewebestrukturen kamen, mithilfe von Kugeldiamanten durchgeführt, um Traumatisierungen umgebener Strukturen auszuschließen.

Wenn die Pulpa nach kaudal so weit eröffnet wurde, dass eine ausreichende Übersicht zur weiteren Trennung bestand, wurde der nächste Schritt, die Odontosektion, durchgeführt. 


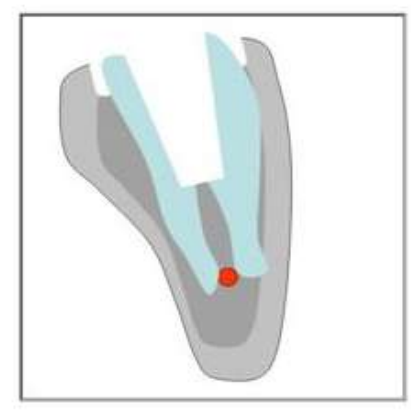

(a)

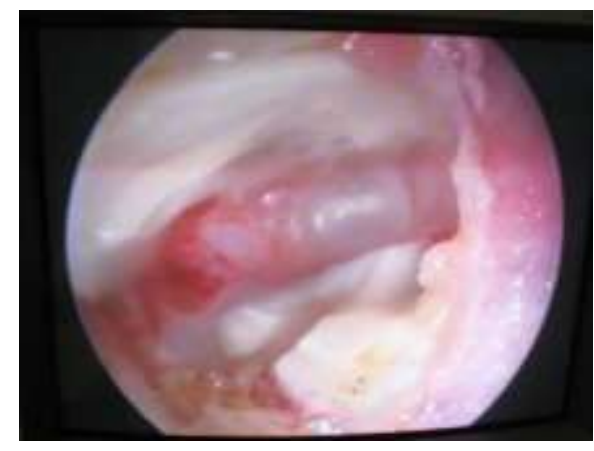

(b)

Abb.2.7: Phase 1 - Raumschaffende Trepanation

a) Schematische Darstellung der raumschaffenden Trepanation

b) Endoskopische Aufnahme der raumschaffenden Trepanation

\section{Phase 2 - Odontosektion}

Die Odontosektion erfolgte, je nach Positionierung des Zahnes, weiter mit Mikroskop oder mit Endoskop. Die Dislokation des Zahnes entschied über die Trennungsrichtung. Es wurde unterschieden zwischen einer Trennung in Richtung der Furkation (bei vertikaler, mesioangulierter, distoangulierter Lage) oder einer Separation zwischen Krone und Wurzel (bei horizontaler Lage). Dabei entstand eine Kavität (erweiterter Pulpenraum), die später als Verfügungsraum der Fragmentmobilisation mittels Implosiontechnik (Luxation in den Innenraum des Zahnes) diente. Wichtig war hierbei die Hilfestellung des Mikroskops oder Endoskops, was eine Separation unter direkter Beobachtung ermöglichte. Mit Anwendung von diamantierten Fräsen konnten kritische Zonen, wie z. B. die linguale Alveolenwand wie auch kanalnahe Bereiche, geschont werden.

In Zonen, die kein großes Gefährdungspotential aufweisen, wie z. B im bukkalen Bereich, konnte der Rosenbohrer verwendet werden. Bei ungünstiger Lage und Größe des Kronenfragments wurden diese durch eine sagittale Trennung zusätzlich soweit reduziert, dass sie ohne weiteren Knochenverlust nach okklusal entwickelt werden konnten.

Anders als bei der konventionellen Technik wurde die Krone bzw. das einzelne Fragment nicht nach außen luxiert, sondern in die durch die 
Trepanation geschaffene Kavität des Zahnes (Implosionstechnik). Eine weitere Opferung der Knochensubstanz ist somit vermeidbar gewesen (Abb.2.8).

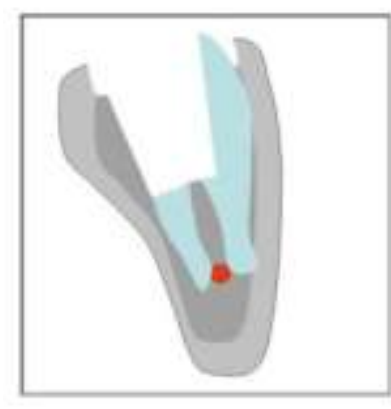

(a)

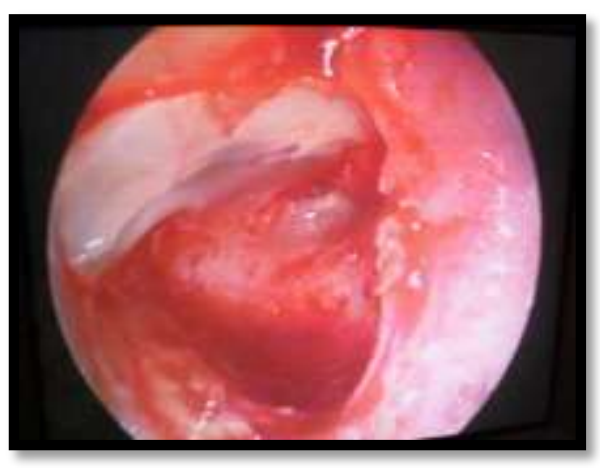

(b)

Abb.2.8: Phase 2 - Odontosektion

a) Schematische Darstellung

b) Endoskopische Aufnahme

\section{Phase 3 - Wurzelentfernung}

Wenn nur noch Wurzelreste in der Alveole verblieben, wurde mit dem Endoskop gezielt unter Sicht gearbeitet. Die Wurzelfragmente konnten mikrochirurgisch zirkulär freigelegt werden, ohne großflächigen Abtrag der bukkalen oder lingualen Alveolenwände. Zur Erleichterung der Entfernung des Wurzelrestes war es sinnvoll, Knochen von der Wurzeloberfläche abzutragen. Wenn weitere Trennungen erforderlich waren, was bei stark gekrümmten Wurzeln oft der Fall war, konnten diese unter endoskopischer Sicht gezielt getrennt werden. Die grazilen Heidbrink-Elevatoren (Hu-Friedy) wie auch Mikroelevatoren dienten zusätzlich dazu, die kleinen Fragmente der Wurzel vorsichtig herauszuluxieren (Abb.2.9).

Für den Fall einer Ankylose wurden die Wurzelanteile unter endoskopischer Sicht mit einem rotierenden Instrument vollständig abgetragen. Das Stützimmersionsendoskop diente hierbei auch zur sicheren Unterscheidung von Wurzeldentin und Knochen, wobei die Volkmann-Kanälchen im Knochengewebe als Unterscheidungskriterium galten. 


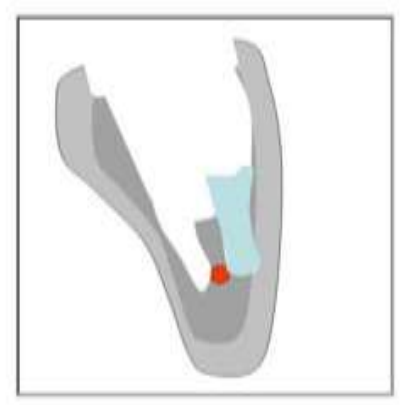

(a)

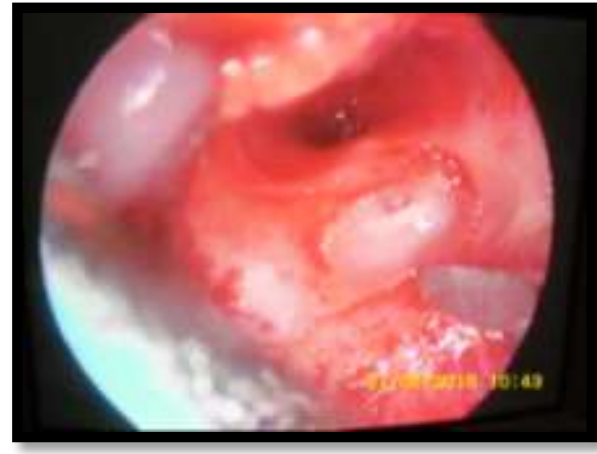

(b)

Abb.2.9: Phase 3 - Wurzelentfernung

a) Schematische Darstellung

b) Endoskopische Aufnahme

\section{Phase 4 - Alveolenmessung}

Zum Abschluss der Operation wurde das Operationsfeld auf Wurzelreste und Reste des Zahnfollikels hin untersucht. Für die Studie wurde im Anschluss mithilfe einer Parodontalsonde die bukkale Knochenhöhe gemessen (Abb.2.10).

Die Wundversorgung erfolgte je nach Ausdehnung der Knochenhöhle mit Kollagenvlies und mit einer nachfolgenden Nahtversorgung (5-0 monofil).

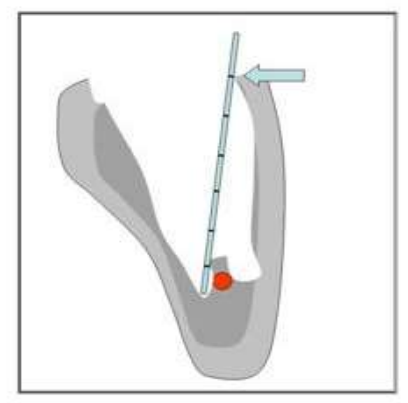

(a)

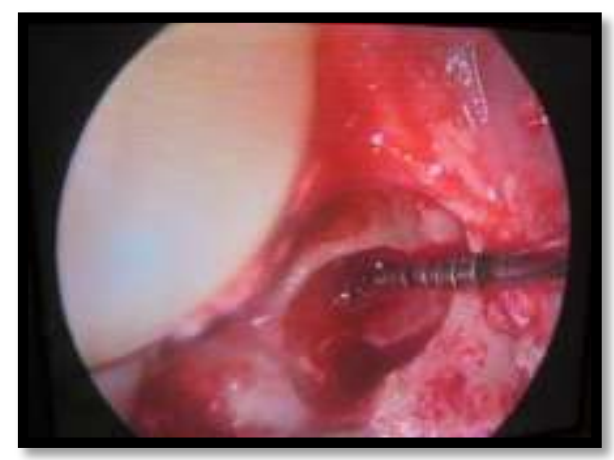

(b)

Abb.2.10: Phase 4 - Alveolenmessung

a) Schematische Darstellung der bukkalen Knochenhöhenmessung

b) Endoskopische Aufnahme der bukkalen Knochenhöhenmessung 


\subsection{Dokumentation der Röntgenbefunde}

Anhand der präoperativ angefertigten Röntgenaufnahmen konnten die Angulationstypen der Weisheitszähne entsprechend der Klassifikation nach Winter (1926) eingeteilt werden.

Dies wurde in Tabellen überführt, um eine Übersicht der Anzahl zu bekommen.

Des Weiteren wurde die Beziehung zwischen dem Weisheitszahn und dem Nervus alveolaris inferior vermessen und beurteilt, ob diese einen Kontakt aufweisen. Die dazu verwendeten Kriterien gleichen denen der Implantologie, bei der ein Sicherheitsabstand von $2 \mathrm{~mm}$ vom Nervus alveolaris inferior eingehalten werden sollte, um eine Nervenschädigung zu vermeiden (Greenstein und Tarnow 2006). In dieser Studie wurde ein Abstand unter $2 \mathrm{~mm}$ als Nervenkontakt dokumentiert.

\subsection{Dokumentation der Operation}

Die gesamte Operation wurde mithilfe des Endoskops und Mikroskops aufgezeichnet. Die endoskopischen Aufzeichnungen erfolgten über eine Videodokumentation und die mikroskopischen Aufnahmen über eine integrierte Software (MediLife Video Tools) in digitalisierter Form. Diese Operationsaufzeichnung wurde später zur Dokumentation verschiedener Parameter ausgewertet.

Die Gesamtdauer der Operation, Operationszeit einzelner Phasen, Beobachtung der Strukturen anhand der Krone und Wurzel, verwendete visuelle Hilfsmittel in einzelnen Phasen, intraoperative Komplikationen und Verwendung der Instrumente wurden in einem Protokoll dokumentiert (Abb.2.11). 


\begin{tabular}{|c|c|c|}
\hline Op - Schritte & Endoskop & Mikroskop \\
\hline \multirow[t]{3}{*}{$\begin{array}{l}\text { 1. Raumschaffende } \\
\text { Trepanation }\end{array}$} & Vorteil: & Vorteil: \\
\hline & Nachteil: & Nachteil: \\
\hline & Bemerkung: & Bemerkung: \\
\hline \multirow[t]{3}{*}{ 2. Odontosektion } & Vorteil: & Vorteil: \\
\hline & Nachteil: & Nachteil: \\
\hline & Bemerkung: & Bemerkung: \\
\hline \multirow[t]{3}{*}{ 3. Wurzel Entfernung } & Vorteil: & Vorteil: \\
\hline & Nachteil: & Nachteil: \\
\hline & Bemerkung: & Bemerkung: \\
\hline 4. Alveolen Messung & Vorteil: & Vorteil: \\
\hline & | Nachteil: & Nachteil: \\
\hline & Bemerkung: & Bemerkung: \\
\hline
\end{tabular}

Abb.2.11: Protokoll zur Dokumentation und Bewertung der Operationsaufzeichnung

\subsection{Auswertung der Knochendefekte}

Um den postoperativen Knochenverlust der bukkalen Alveolenwand zu messen, wurde in dieser Studie das präoperative Röntgenbild mit der intraoralen Knochenhöhenmessung verglichen.

\subsubsection{Präoperative Vermessung des Röntgenbildes}

Um die Messung im Röntgenbild jederzeit reproduzierbar zu gestalten, wurde ein Referenzpunkt, hier der zweite Molar, fest definiert (Abb.2.12). 
Es wurde zuerst eine Tangente am Kronenrand des zweiten Molaren angelegt, die im Wesentlichen der Okklusionsebene entspricht. Anschließend wurde durch diese Kronentangente (kt) wie auch durch den Furkationspunkt $(F)$ des zweiten Molaren eine Senkrechte gezogen, die als Mittellinie $(m)$ dienen soll. An der Knochenkontur entlang wurde die entsprechende Linie nachgezeichnet und als Knochenkonturlinie (kl) definiert.

Am höchsten Punkt des dritten Molaren sowie am tiefsten, bzw. bei horizontal liegenden Zähnen am distalsten Punkt, wurden jeweils Punkt $\mathrm{H}$ und Punkt $A$ bzw. Punkt $D$ definiert. Parallel zur Linie kt konnte durch Punkt H (hp) wie auch durch Punkt A (ap) eine Linie senkrecht zur Mittellinie gezogen werden, die als Referenzlinie bei weiteren Vermessungen dienen konnte. Die Strecken senkrecht zur Mittellinie konnten dokumentiert und bei erforderlichen Mess-Wiederholungen verwendet werden.

Als nächstes wurde eine Senkrechte parallel zur Mittellinie durch Punkt $H$ gezogen (Ih), wobei der Schnittpunkt durch Linie ap als $A^{\prime}$ definiert wurde. Dieser Punkt diente zur Bestimmung der Zahnlänge (H-A, H-A').

Zuletzt wurde der Knochenpunkt (K) bestimmt, der am Schnittpunkt der Linie Ih und $\mathrm{kl}$ entstand. Von diesem Punkt $(\mathrm{K})$ wurde die Alveolenlänge $(\mathrm{K}$ $A^{\prime}$ bzw. K-D) vermessen. Des Weiteren konnte die Strecke K-H, bezogen auf den Impaktierungsgrad des Zahnes, Informationen geben.

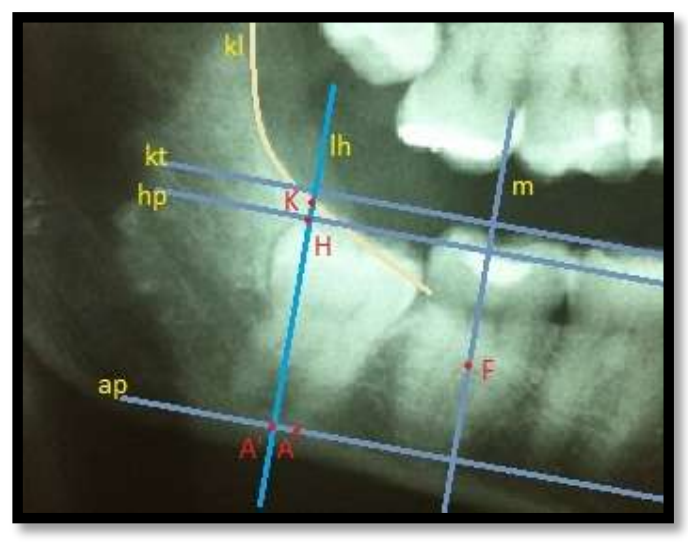

(a)

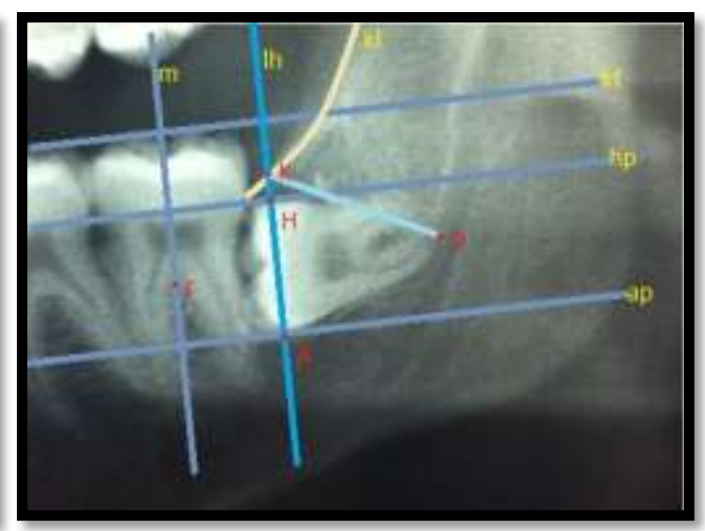

(b)

Abb.2.12: Röntgenologische Messung am (a) vertikalen und (b) horizontalen Weisheitszahn 


\title{
Punkte
}

F : Furkationspunkt $\mathrm{M}_{2}$

H : höchster Punkt von $\mathrm{M}_{3}$

A : tiefster Punkt von $\mathrm{M}_{3}$ (vertikal)

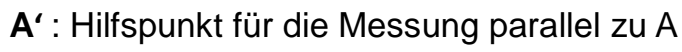

K : Knochenpunkt - Schnittpunkt von Linie Ih und kl

D : tiefster Punkt der Alveole in der Zahnlängsachse

\author{
Linien \\ kt : Kronentangente des $\mathrm{M}_{2}$ \\ $\mathbf{m}$ : Mittellinie des $\mathrm{M}_{2}$ \\ kl : Knochenlinie \\ hp : Parallele zu kt durch den Punkt $H$ \\ ap : Parallele zu kt durch den Punkt $A$ \\ Ih : Lot von kt-linie durch $\mathrm{H}$
}

\section{Strecken}

H-A bzw. H-A' : Zahnlänge

K-D bzw. K-A': Alveolenlänge

K-H : Distanz zwischen Knochenhöhe und Zahnlänge (positiv/negativ), Impaktierungsgrad

\subsubsection{Intraoperative Vermessung der Alveolenwand}

Mithilfe des Endoskops oder Mikroskops wurde der Knochendefekt vom tiefsten Punkt der Alveole bis zum höchsten Punkt der bukkalen Alveolenwand vermessen (Abb.2.13). Da bei den horizontal angulierten Zähnen die tiefste Stelle oft in der horizontalen Ebene liegt, wurde hier der distalste Punkt in der Alveole gemessen. Als Messinstrument wurde eine Parodontalsonde der Firma Hu-Friedy verwendet, die eine Skalierung im mm-Abstand aufwies.

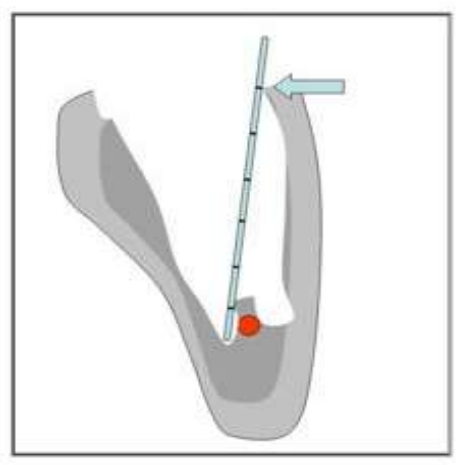

Abb.2.13: Schematische Darstellung der intraoralen Vermessung des Knochendefektes nach Entfernung des Weisheitszahnes 


\subsubsection{Betrachtung der Messfehler}

Da verschiedene Faktoren eine zweidimensionale Röntgenbilddarstellung der Knochensituation und eine dreidimensionale Intraoralmessung beeinflussen können, wurden diese geometrischen und/oder dimensionalen Fehlerfaktoren ausgerechnet und behoben (Abb.2.14).

Mithilfe eines extrahierten Weisheitszahnes wurde mit dem Abdruckmaterial (Silaplast) die Alveole nachkonstruiert. Dieser Abdruck wurde in der Mitte geteilt und ohne den Zahn wieder zusammengefügt, sodass eine nachgestellte Extraktionsalveole entstehen konnte.

Die Berechnung erfolge anhand der Bestimmung des Angulationsunterschiedes zwischen rechtwinkeliger und leicht geneigter Positionierung der Messsonde, die maximal bis zur bukkalen Alveolenwand reichen konnte.

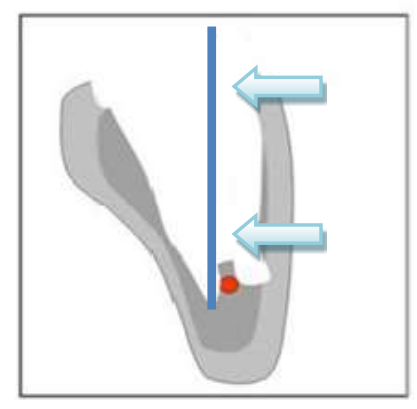

(a)

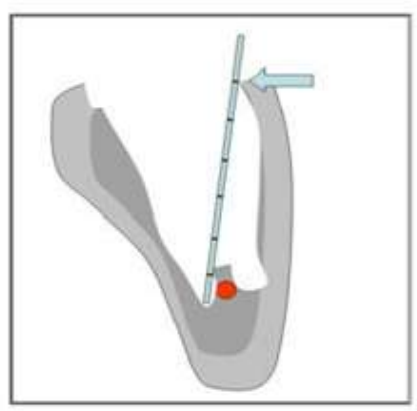

(b)

Abb.2.14: Schematische Darstellung der Fehlerfaktoren, die durch geometrische Umstellungen in der Vermessung verursacht werden konnten

(a) Zähne, die senkrecht zur Alveolarkammoberfläche gemessen werden (entspricht der röntgenologischen Vermessung)

(b) Zähne, die zum höchsten Punkt der bukkalen Alveolenwand, transversal leicht gekippt gemessen werden. (entspricht der intraoralen Vermessung)

Roter Punkt : Nervus alveolaris inferior

Blaue Linie : Senkrechte Messung

Pfeil : (a) Strahlengang (b) Messung an der bukkalen Alveolenwand

Dabei entstand aufgrund des Neigungsunterschiedes der Parodontalsonde 
ein Messunterschied von max. $20 \mathrm{Grad}$. Die Differenz wurde mithilfe des Hypotenusensatzes berechnet, um den Fehlerfaktor zu ermitteln (Abb.2.15).

Strecke ac (angulierte $\left.M_{.}\right) \times \cos 20^{\circ}=$ Strecke bc (senkrechte $\left.M_{\text {. }}\right)$ (intraorale Messung) (röntgenologische Messung)
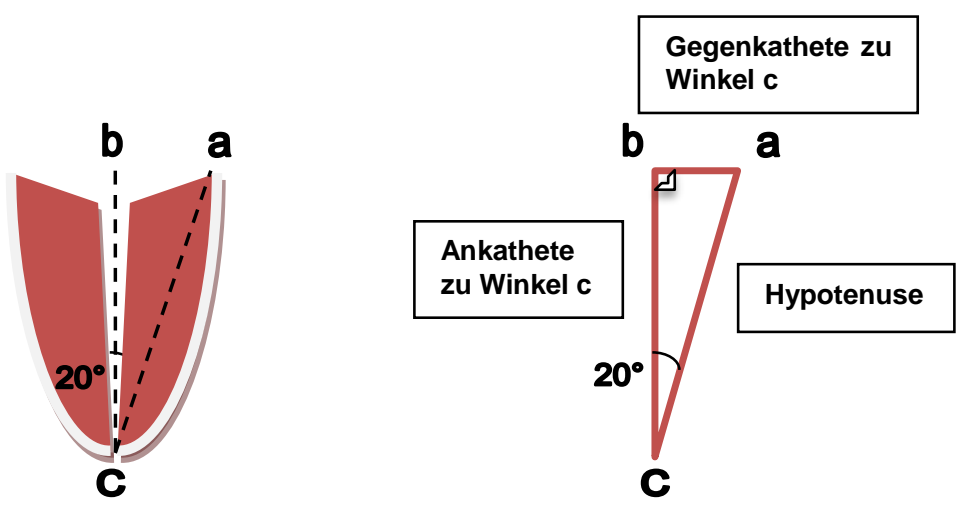

Abb.2.15: Schematische Darstellung der leeren Alveole im Sagittalschnitt

a: Punkt an der höchsten Stelle der bukkalen Alveolenwand

b: Punkt an der Knochenkonturlinie der Alveole im Röntgenbild

c: Punkt an der tiefsten Stelle der Alveole

a-c: Länge vom Apex bis zur bukkalen Alveolenwand (Hypotenuse)

b-c: Länge vom Apex bis zur Knochenkonturlinie im Röntgenbild (Ankathete zu Winkel c)

Die maximale Differenz, ein Vergrößerungsfaktor von $7 \%$, die zwischen beiden Messungen entstand, wurde von der intraoralen Messung subtrahiert.

Zusätzlich wurden alle Röntgenbilder um den Vergrößerungsfaktor von 1,25 korrigiert. 


\section{Ergebnisse}

\subsection{Patientenkollektiv}

Das in der Studie untersuchte Gesamtkollektiv beläuft sich auf 40 Patienten mit insgesamt 50 Unterkieferweisheitszähnen, 26 auf der linken und 24 auf der rechten Seite (Abb.3.1). Die Zähne wurden bei den Patienten nach rechtfertigender Indikation uni- oder bilateral operativ entfernt. Unmittelbar nach der Entfernung wurden die Extraktionsalveolen untersucht und vermessen.

Es handelte sich um 19 männliche und 21 weibliche Patienten (Abb.3.1), die im Alter von 15-55 $(27,27)$ Jahren behandelt worden sind (Abb.3.2). Die gesamte Studie erfolgte im Zeitraum von April 2010 bis Juni 2011.

Geschlechterverteilung

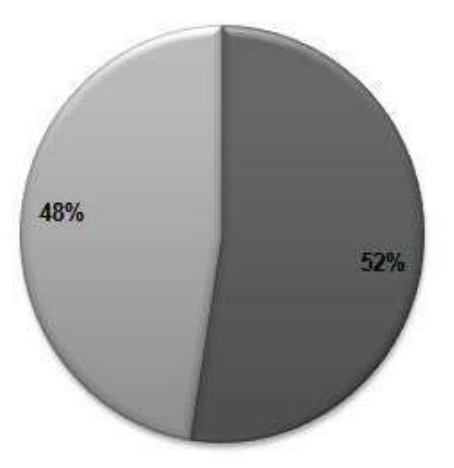

\section{Zahnverteilung}

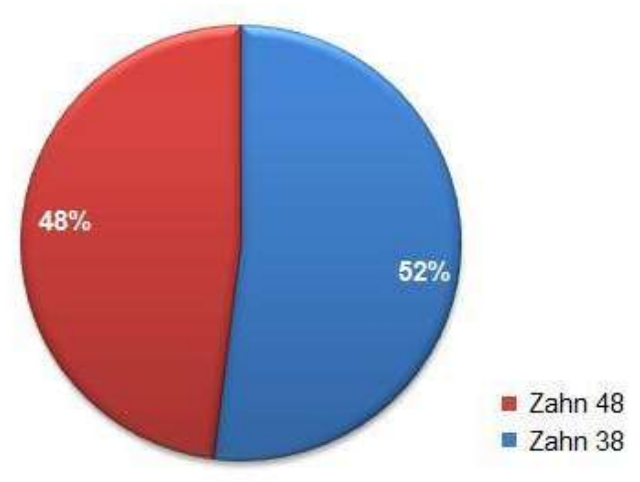

Abb.3.1: Schematische Darstellung der Geschlechterverteilung und Zahnverteilung im Unterkiefer 


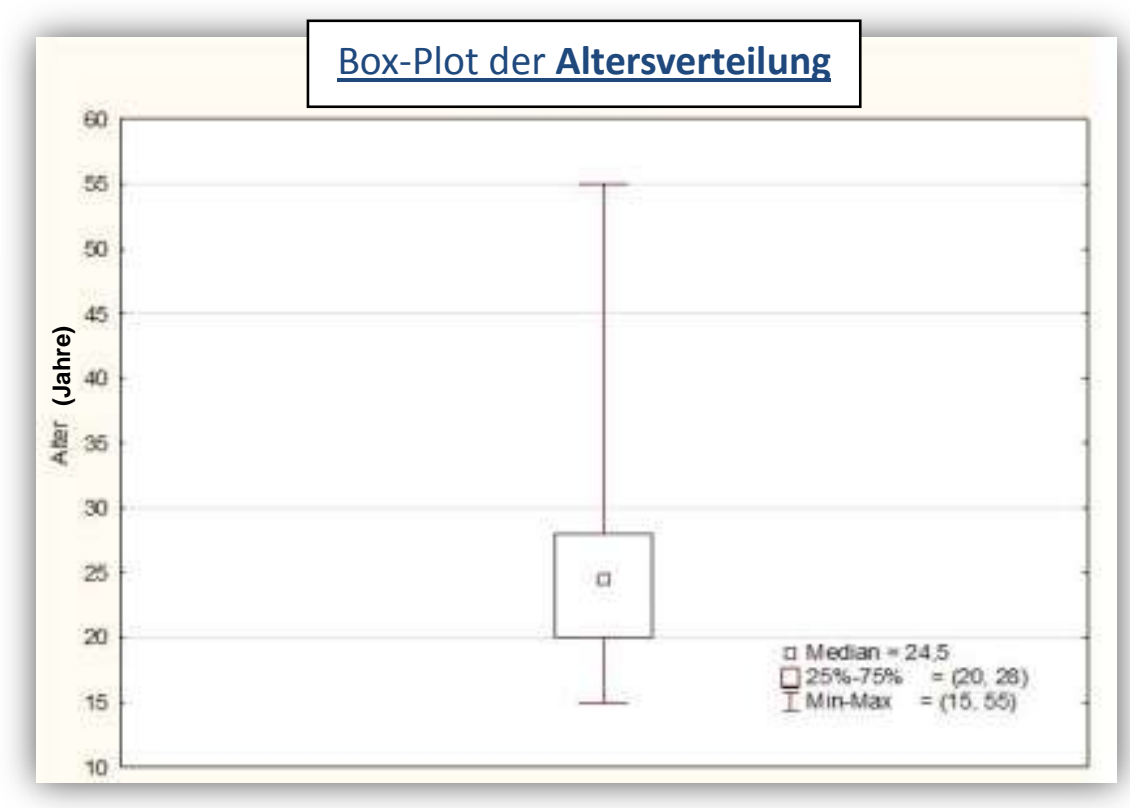

Abb.3.2: Box-Plot Darstellung der Altersverteilung vom Patientenkollektiv

Des Weiteren wurde mittels präoperativer Röntgenbildern die Angulation der einzelnen Weisheitszähne ermittelt und anhand der Klassifikation nach Winter (1926) unterteilt.

Von 50 Weisheitszähnen konnten 15 vertikal (30\%), jeweils 14 mesial (28\%) und horizontal (28\%) und 7 distal (14\%) anguliert, eingeteilt werden. Transversal angulierte Zähne wurden bei den untersuchten Patienten nicht identifiziert (Abb.3.3). 


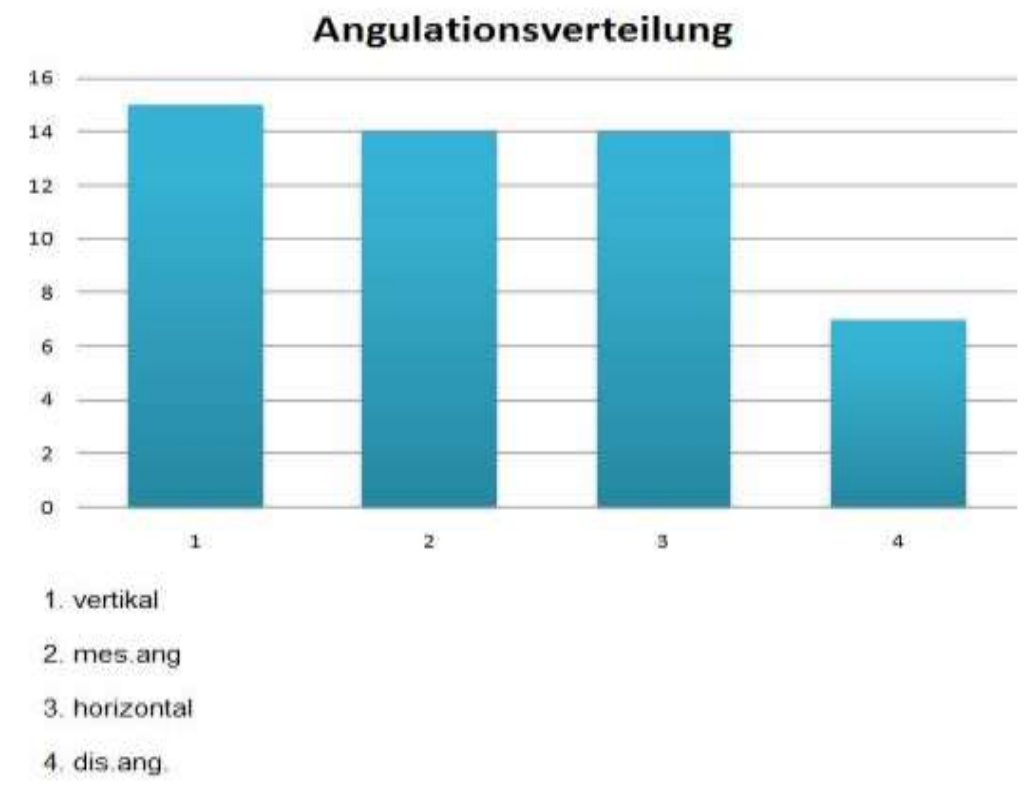

Abb.3.3: Grafische Darstellung der Angulationsverteilung anhand der Klassifikation nach Winter (1926)

\subsection{Endoskop und Mikroskop}

\subsubsection{Beobachtung der Strukturen}

Die Aufzeichnung der gesamten Operation, die mit Aufnahmen durch Mikroskop und Endoskop erfolgte, wurde zur Bewertung der Übersichtlichkeit des Operationsfeldes genutzt. Anhand der sichtbaren Strukturen, wie der Krone und der Wurzel, konnte eine Beurteilung der visuellen Hilfsmittel dargestellt werden (Tab.3.1).

\begin{tabular}{cccc}
\hline \multicolumn{2}{c}{ Mikroskop } & \multicolumn{2}{c}{ Endoskop } \\
\hline Krone & Wurzel & Krone & Wurzel \\
$88 \%$ & $50 \%$ & $12 \%$ & $50 \%$ \\
\hline
\end{tabular}

Tab.3.1: Tabellarische Darstellung der optischen Bewertung von anatomischen Strukturen 
Das Ergebnis zeigte, dass die Darstellung der Strukturen im Kronenniveau überwiegend mit dem Mikroskop beobachtet wurde, was sich anhand der häufigen Anwendung (88\%) äußerte. Die Strukturen, die im Wurzelniveau beobachtet werden konnten, wurden mit jeweils 50\% anhand des Mikroskops sowie auch Endoskops gleich gut beobachtet.

\subsubsection{Anwendung}

In einem Protokoll wurde die Operation dokumentiert und ausgewertet, wobei die Anwendung der visuellen Hilfsmittel (Endoskop und Mikroskop) in den verschiedenen Phasen ermittelt werden konnte.

Hierbei wurden die vier Phasen raumschaffende Trepanation, Odontosektion, Wurzelentfernung und Alveolenmessung festgehalten. Die vier verschiedenen Abbildungen zeigen, wie häufig die Anwendung von Endoskop und Mikroskop oder von beiden zusammen erfolgte. Die makroskopische Sicht, wobei keinerlei mikro-/endoskopische Hilfsmittel verwendet worden sind, wurde in der Untersuchung als Sonstiges bezeichnet (Abb.3.4).

In der ersten Phase überwog die Anwendung vom Mikroskop mit 44\%, gefolgt vom Endoskop mit 22\%, Mikroskop/Endoskop mit 18\% und $16 \%$ Sonstiges. In der zweiten Phase überwog die Anwendung vom Endoskop mit 46\%, wobei die Anwendung vom Mikroskop mit 38\% im Vergleich zur ersten Phase etwas abgenommen hat. Die kombinierte Anwendung von beiden Geräten erfolgte deutlich seltener (8\%). In der dritten Phase blieb das Endoskop mit $40 \%$ das am meisten verwendete Hilfsmittel. Die Anwendung vom Mikroskop sowie auch die Kombination von beiden Geräten blieben mit jeweils $38 \%$ und $8 \%$ gleich. In der vierten Phase gab es erneut eine deutliche Steigerung der endoskopischen Anwendung mit $56 \%$. $18 \%$ der Operationen erfolgten in diesem Abschnitt mit mikroskopischer Unterstützung. Eine Kombination blieb in dieser Phase ausgeschlossen.

Eine Zuordnung der visuellen Hilfsmittel zu verschiedenen Angulationstypen der Weisheitszähne konnte nicht ermittelt werden (keine Signifikanz 
feststellbar: $p \geq 0,05$ ).

Es gab lediglich eine Tendenz, dass beim Fortschreiten der Phasen mehr endoskopische Unterstützung in Anspruch genommen wurde.
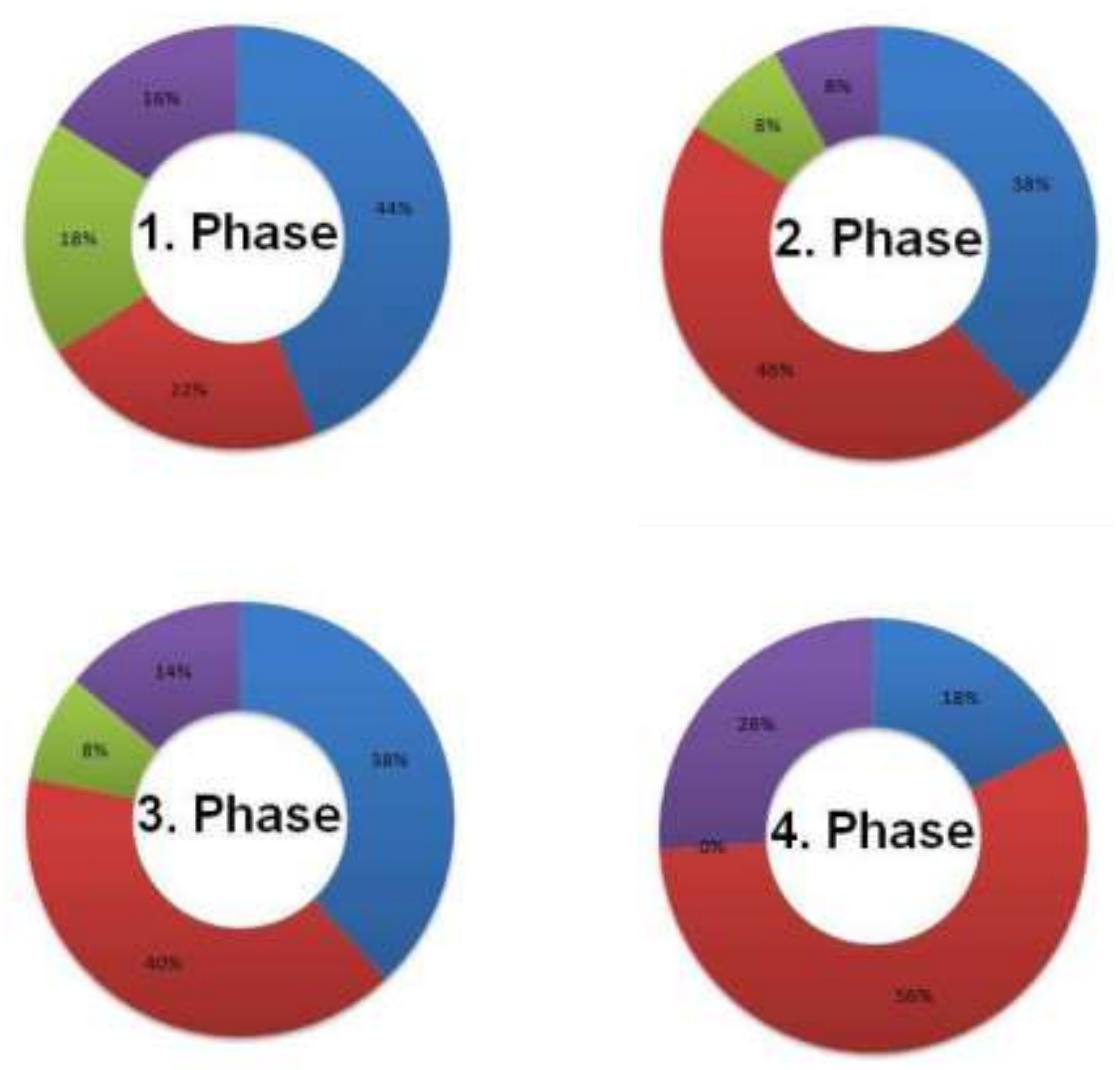

$\mathbf{a} \mathbf{m}$

$=2 \mathbf{e}$

w 3 mie

$=4$ sonstiges

Abb.3.4: Schematische Darstellung der Anwendung von Mikroskop und Endoskop in den vier Phasen

\subsection{Operationszeit}

Zur Beurteilung der Operationszeit wurden die letzten vier Phasen (raumschaffende Trepanation, Odontosektion, Wurzelentfernung, Alveolenmessung) der Operation sowie auch die Gesamtdauer der vier Phasen 
ausgewertet (Tab.3.2). Die verschiedenen Retentionsarten der Weisheitszähne verlangten unterschiedliche Vorgehensweisen, worauf der impaktierte Zahn erst mittels Osteotomie freigelegt wurde und der teilretinierte Zahn sofort trepaniert werden konnte. Um diese Variablen zu vermeiden, wurde die Zeit der Inzision und Osteotomie vernachlässigt.

In der Abb.3.5 erfolgte die Darstellung der Zeitverteilung in den vier Phasen. Im Durchschnitt zeigten die vier Phasen eine Durchführungszeit von ca. $346,048 \mathrm{~s}$ (5.76 min), $399.978 \mathrm{~s}$ (6.67 min), 352,581 s (5.87 min) und $52 \mathrm{~s}$, wobei die Streuung der einzelnen Fälle im Box-plot Diagramm deutlicher wurde.

Die erste Phase zeigte eine Streuung von $42 \mathrm{~s}$ bis zu $900 \mathrm{~s}$ (15 min) und die zweite Phase von $20 \mathrm{~s}$ bis zu $1874 \mathrm{~s}$ (30,78 min). Die dritte Phase variierte in der Zeit am meisten mit einer Zeit von $40 \mathrm{~s}$ bis zu ca. $3210 \mathrm{~s}$ (53 min). Im Gegensatz dazu war in der vierten Phase eine geringe Streuung festzustellen, von $18 \mathrm{~s}$ bis $101 \mathrm{~s}$ (1,62 min).

Die Gesamtdauer im Durchschnitt betrug 1105,675 s (18,41 min). Auch hier gab es in der Streuung einen Unterschied von minimal $216 \mathrm{~s}$ (3,6 min) bis zu maximal $5372 \mathrm{~s}(89,5 \mathrm{~min})$ (Abb.3.6).

\begin{tabular}{|c|c|c|c|c|}
\hline & valid N & mean & minimum & maximum \\
\hline Phase1 (Zeit in sek.) & 42 & 346,048 & 42 & 900 \\
\hline Phase2 (s) & 46 & 399,978 & 20 & 1847 \\
\hline Phase3 (s) & 43 & 352,581 & 40 & 3210 \\
\hline Phase4 (s) & 44 & 52 & 18 & 101 \\
\hline Gesamt OP. (s) & 40 & 1105,675 & 216 & 5372 \\
\hline
\end{tabular}

Tab.3.2: Tabellarische Darstellung der Operationszeiten 


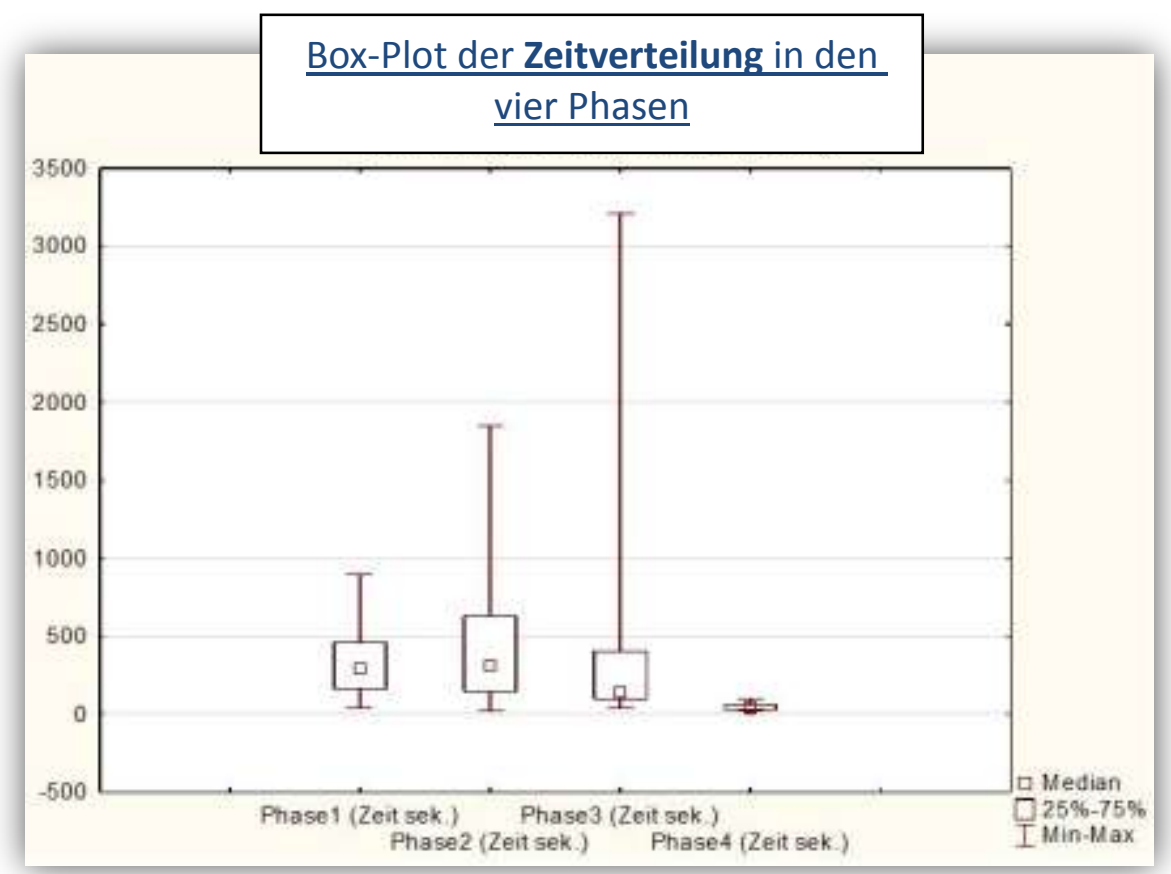

Abb.3.5: Box-Plot Darstellung der Zeitverteilung in den vier Phasen

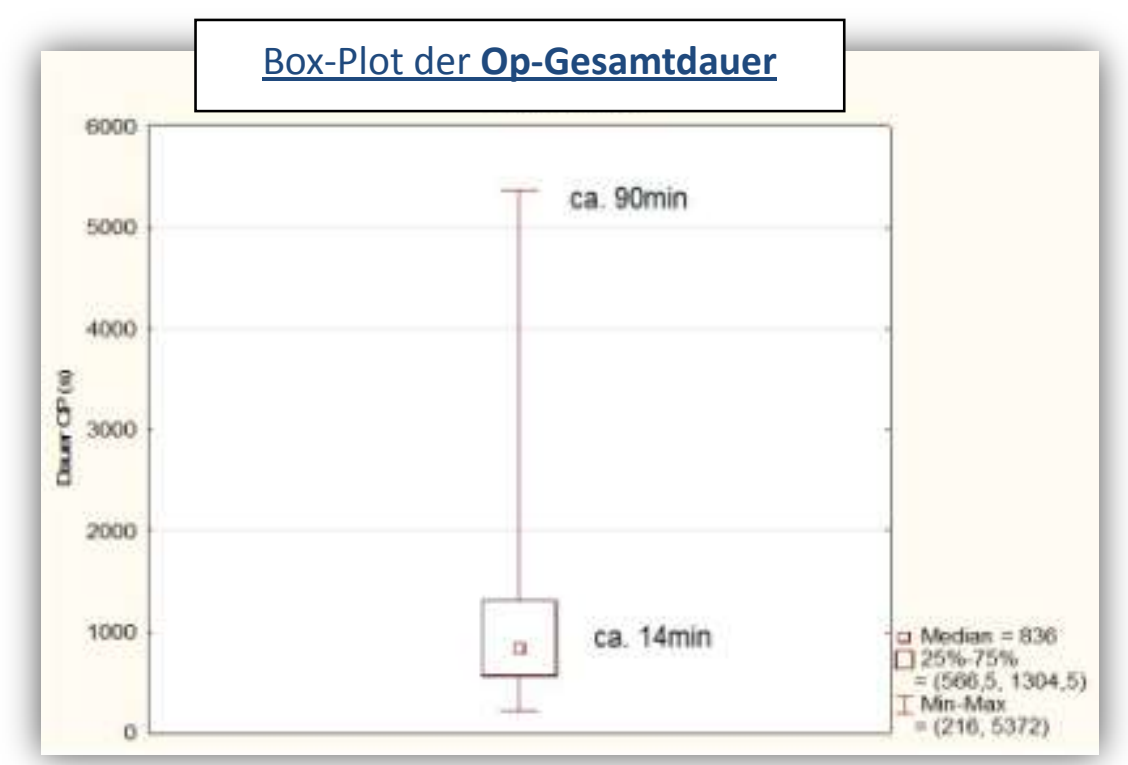

Abb.3.6: Box-Plot Darstellung der Op-Gesamtdauer

Weiterführend wurde in dieser Studie auch die Operationszeit in Korrelation zu den verschiedenen Angulationstypen ermittelt, um einen Zusammen- 
hang zwischen der Lage des Zahnes und der Operationszeit zu erfassen. Dabei stellte sich kein signifikanter Unterschied zwischen den vier Angulationstypen heraus $(p \geq 0,05)$.

Es ist eine Tendenz erkennbar gewesen, dass im Durchschnitt die vertikal angulierten Zähne die längste Operationszeit (22,58 min) in Anspruch genommen haben, gefolgt von den horizontal (21,55 min), mesial (16,11 min) und distal (12,10 min) angulierten Zähnen (Abb.3.7).

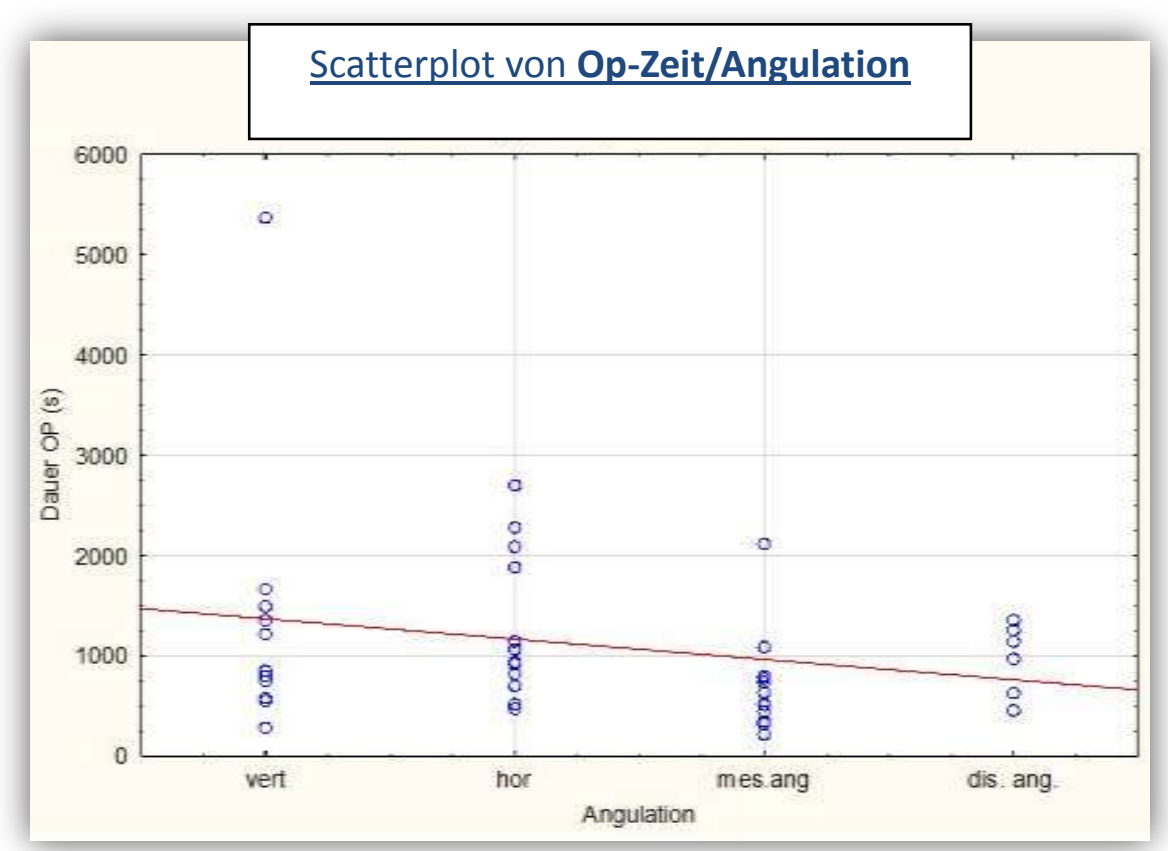

Abb.3.7: Grafische Darstellung der Angulation im Zusammenhang mit der Operationszeit

Rote Linie : Verbindungslinie der Durchschnittswerte der Operationszeit

\subsection{Knochendefektsituation}

In der vorliegenden Studie wurde anhand der präoperativen Röntgenbilder und der intraoperativen Alveolarwandmessungen die bukkale Knochenhöhe prä- und postoperativ bei 40 Zähnen verglichen. Die Auswertung erfolgte unter standardisierten Bedingungen. 
Die bukkale Alveolenwand wies präoperativ im Durchschnitt eine Knochenhöhe von 14,9 mm an, wobei eine Streuung zwischen $10 \mathrm{~mm}$ bis $19,5 \mathrm{~mm}$ feststellbar war. Postoperativ wurde eine durchschnittliche Knochenhöhe von 12,9 mm gemessen, mit einer Streuung von $8,7 \mathrm{~mm}$ bis 17,3 mm (Abb.3.8).

Der Vergleich der Knochendefekte zeigte prä- und postoperativ eine Differenz von minimal $0,4 \mathrm{~mm}$ bis maximal $6,5 \mathrm{~mm}$.

Letztendlich wurden im Durchschnitt ca. $2 \mathrm{~mm}$ Knochenhöhenverlust festgestellt. Der einzelne Vergleich des Knochendefektes aller untersuchten Zähne wird in Abb.3.9 dargestellt.

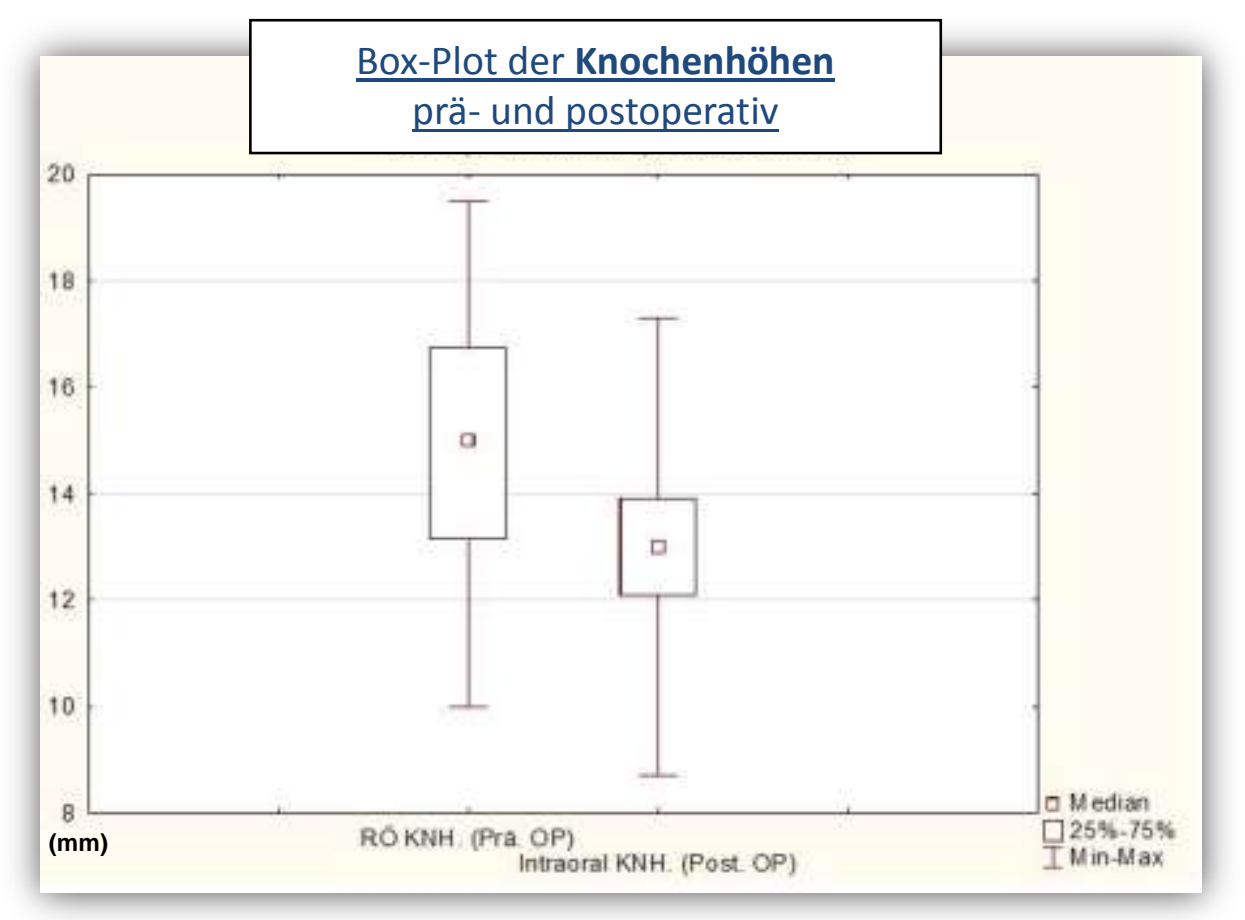

Abb.3.8: Box-Plot Darstellung der Knochendefektsituation der bukkalen Alveolenwand prä- und postoperativ (KNH: Knochenhöhe) 


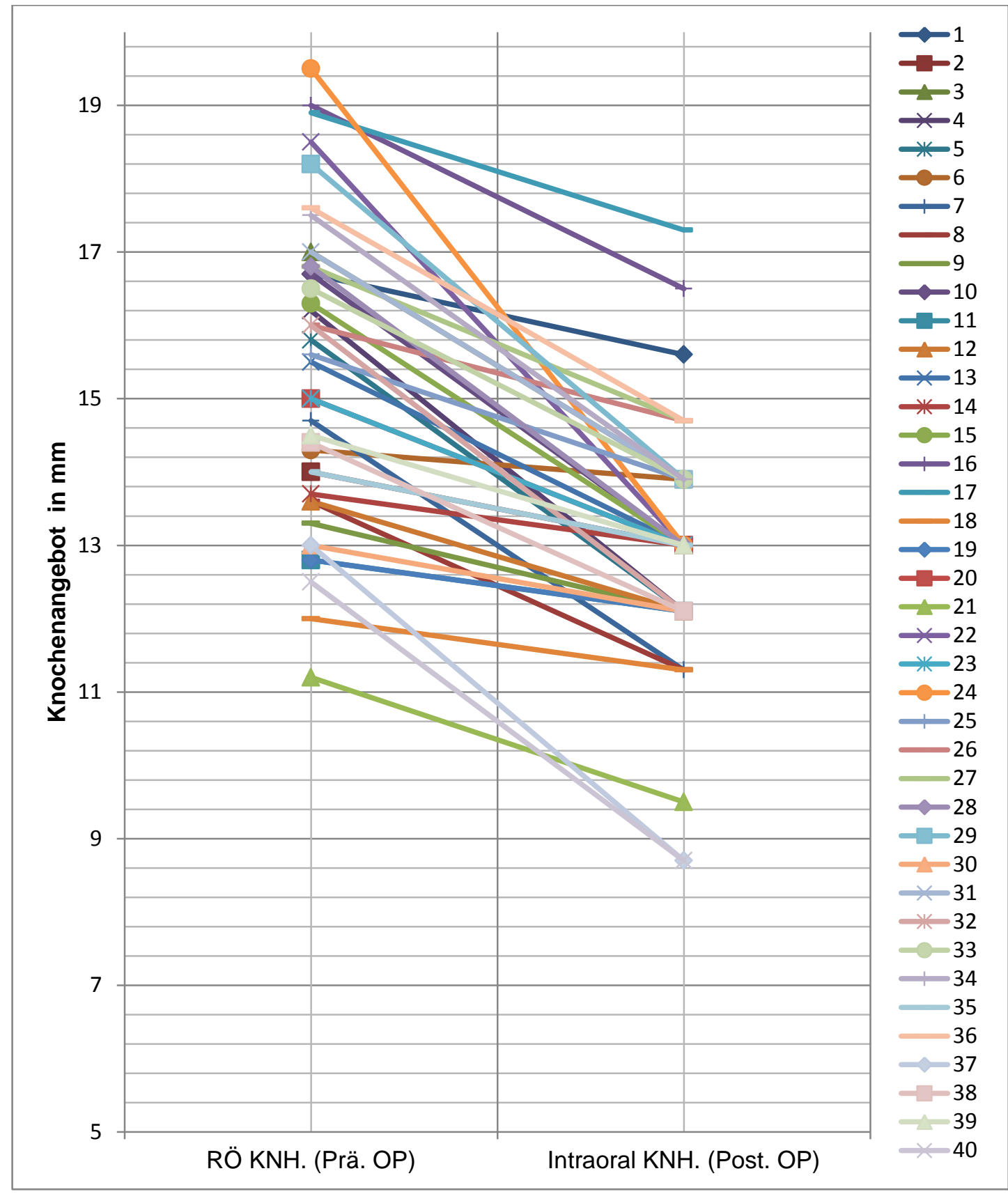

Abb.3.9: Schematische Darstellung der Knochenverlustsituation der bukkalen Alveolenwand prä- und postoperativ: Vergleich aller vermessenen Weisheitszähne (KNH: Knochenhöhe)

Die Knochendefekte wurden auch in Korrelation mit den Angulationstypen ausgewertet, wobei keine signifikanten Unterschiede festgestellt werden konnten $(p \geq 0,05)$. Abb.3.10 zeigt einen relativ konstanten Knochenverlust im Bereich von ca. 2-3 $\mathrm{mm}$ bei den vier verschiedenen Angulationstypen. 


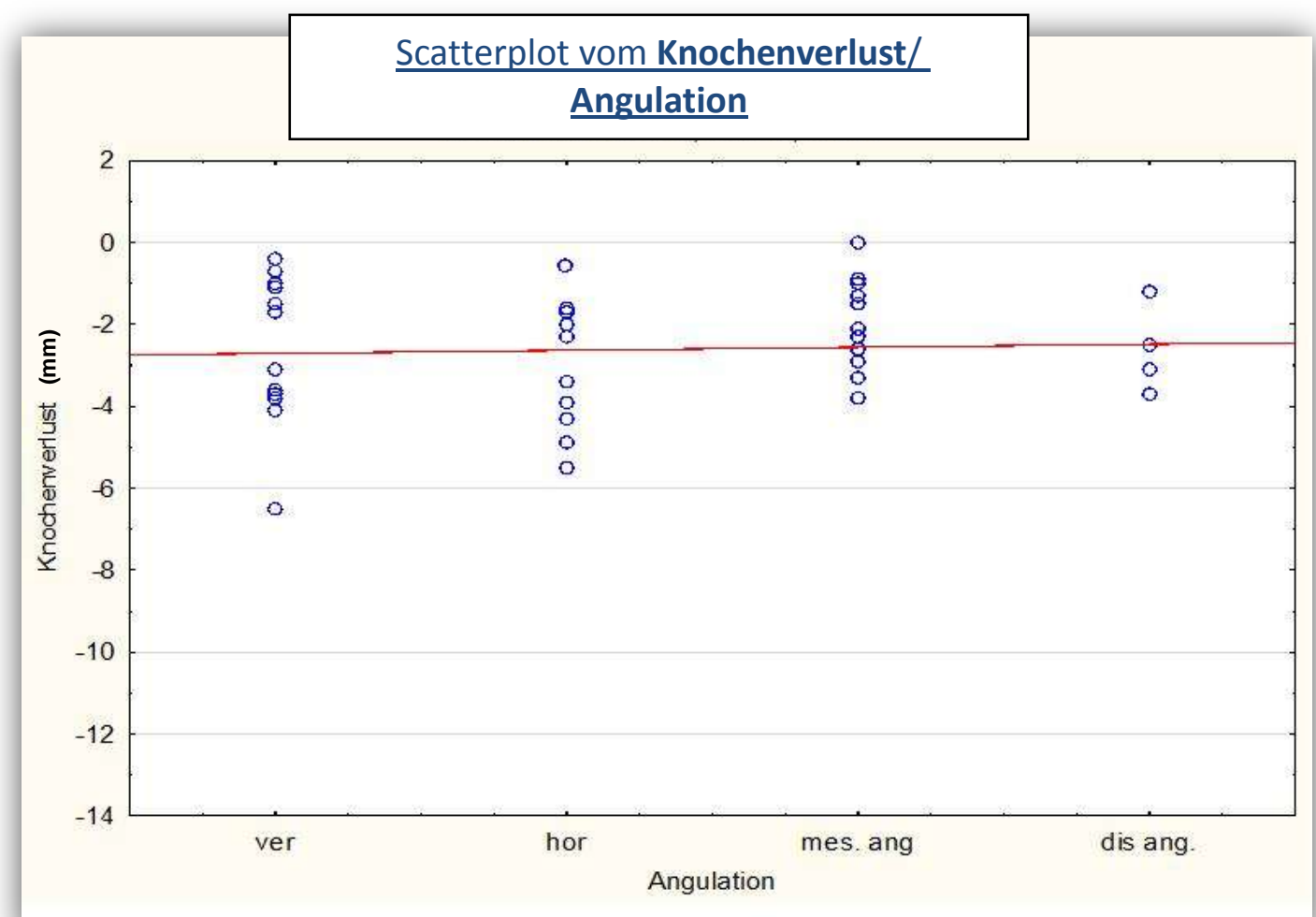

Abb.3.10: Grafische Darstellung des Knochenverlustes im Zusammenhang mit den Angulationstypen

Rote Linie: Verbindungslinie des Durchschnittsknochenverlustes der vier Angulationstypen

\subsection{Komplikationen}

Anhand intraoperativer Aufzeichnungen wie auch der Patientenakten erfolgte die Dokumentation der aufgetretenen Komplikationen.

Die Auswertung zeigte intraoperativ keine auffälligen Komplikationen, wobei postoperative Komplikationen festgestellt werden konnten.

92\% der entfernten Zähne wiesen eine enge Beziehung mit dem Nervus alveolaris inferior auf, was als Risikofaktor einer Nervenläsion betrachtet wurde (Benediktsdottir et al. 2004) (Tab.3.3). 


\begin{tabular}{cccc}
\hline Nervenkontakt & Anzahl & Prozent (\%) & Komplikation \\
\hline Ja & 46 & 92 & 6 \\
Nein & 4 & 8 & 0 \\
\hline
\end{tabular}

Tab.3.3: Tabellarische Darstellung der Zähne, die eine enge Beziehung zum Nervus alveolaris inferior aufweisen

Die Anzahl der Komplikationen, die postoperativ diagnostiziert werden konnten, zeigten sich in sechs Fällen (12\%). 44 Fälle (88\%) blieben unauffällig (Abb.3.11).

Die Mehrzahl der aufgetretenen Komplikationen waren Hyp-, wie auch Parästhesien $(6 \%)$, die postoperativ befundet werden konnten. Alle Sensibilitätsstörungen waren in späteren Nachuntersuchungen vollständig reversibel.

Postoperative Entzündungen wurden als zweithäufigstes beobachtet (4\%), die sich durch Rötung, Schwellung, Schmerz und Eiterbildung äußerten. Außerdem gab es einmalig eine Indikation zur Revision aufgrund eines Wurzelrestes. Dieses wurde durch postoperative Entzündungs-zeichen und anhand eines Röntgenbildes befundet.

Eine signifikante Zuordnung zu anderen Parametern wie Angulationstyp und Operationsdauer (Abb 3.12) konnte nicht festgestellt werden $(p \geq 0,05)$. 


\section{postoperative Komplikationen}

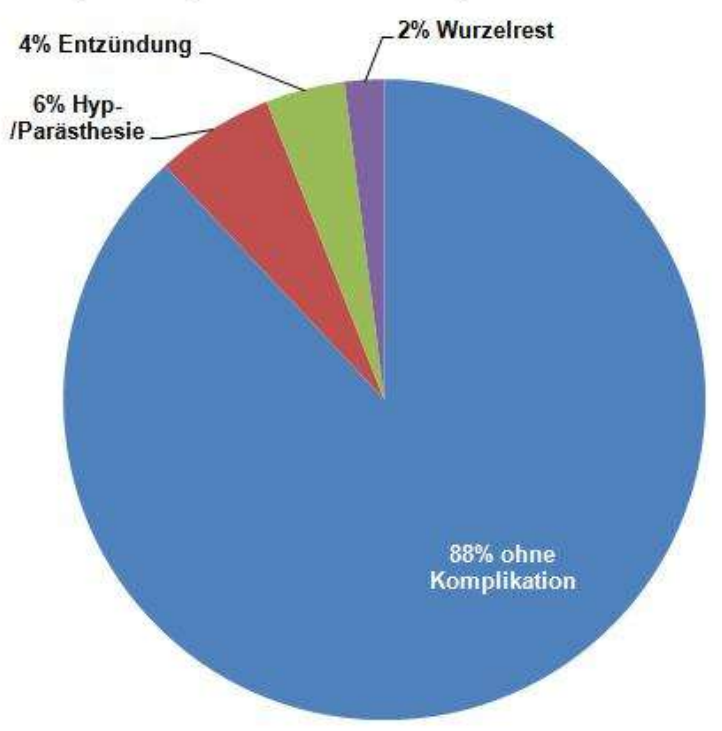

Abb.3.11: Schematische Darstellung der postoperativen Komplikationen

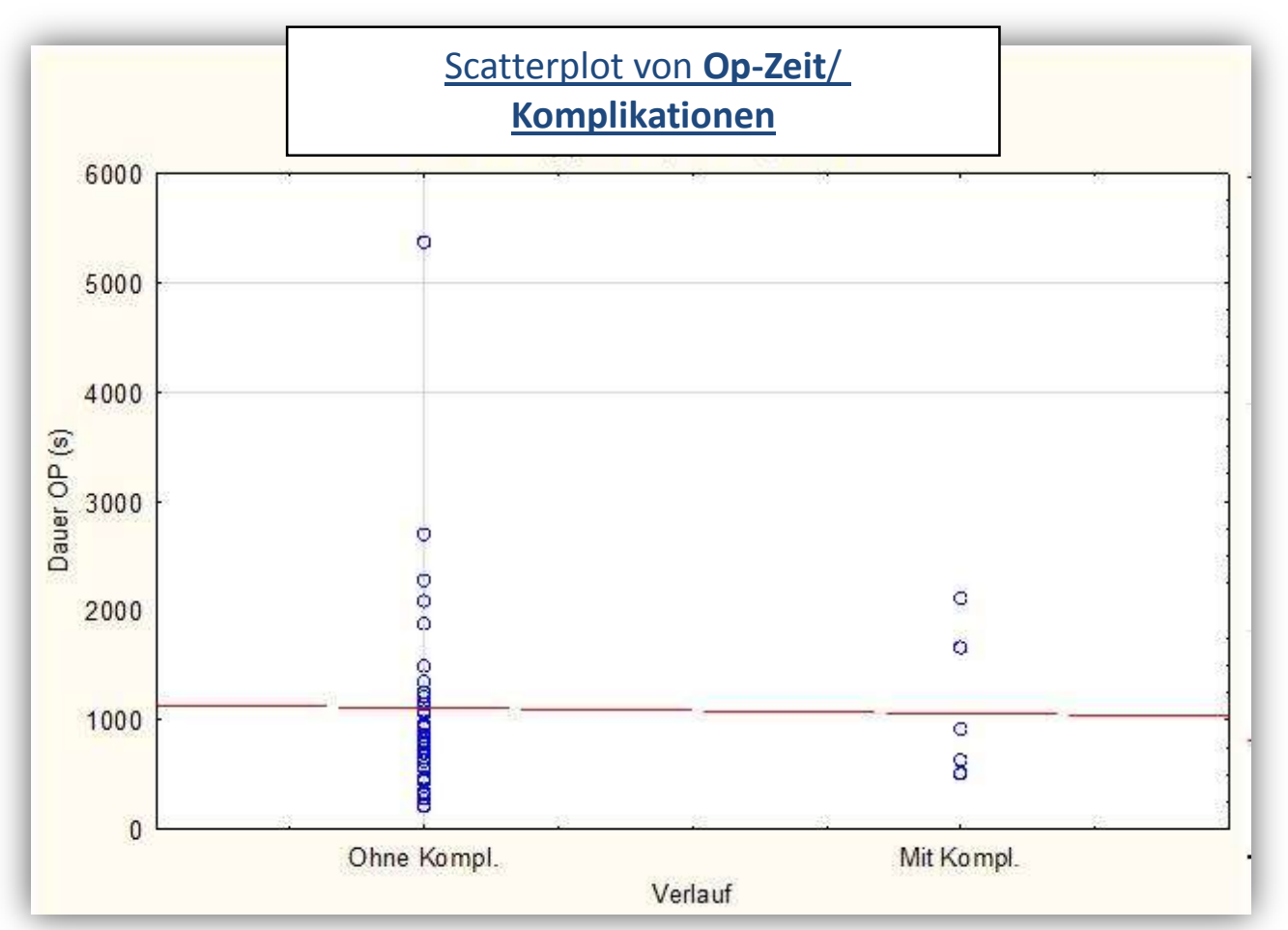

Abb.3.12: Schematische Darstellung der Komplikationen im Zusammenhang mit der Op-Zeit

Rote Linie: Verbindungslinie der Durchschnittszeiten der Operation mit und ohne Komplikation 


\section{Diskussion}

In vielen Disziplinen der Medizin haben minimal-invasive Verfahren Einzug gehalten und etablieren sich derzeit auch bei zahnmedizinischen Routineeingriffen wie der Weisheitszahnentfernung. Vor allem die Vermeidung intra- und postoperativer Komplikationen sowie die Verhinderung von großen Weich- und Hartgewebsdefekten sind das Ziel bei neueren Untersuchungen. Beispiele für die Optimierungen der chirurgischen Entfernung dritter Molaren stellen die Flapless-Inzision, zweizeitige Operation sowie die Trennung der Weisheitszähne dar (Kim et al. 2011; Pogrel et al. 2004; Tolstunov et al. 2011).

Die vorliegende Studie überprüft ebenfalls eine neue minimal-invasive Vorgehensweise bei der Weisheitszahnosteotomie auf ihre Anwendungstauglichkeit.

\subsection{Technische Bewertung}

\subsubsection{Durchführbarkeit}

In der Literatur werden verschiedene Verfahren der Weich- und Hartgewebspräparationen vorgestellt, die im einleitenden Kapitel schon zum Teil erwähnt wurden. Der Versuch, möglichst atraumatisch zu operieren wurde schon seit langem, teilweise mit guten Ergebnissen, untersucht (Ward 1956, Kim et al. 2011).

Die Idee, gewebeschonend zu arbeiten, ging erstmals in die Technik der Inzision ein, die sich über die Jahre von der konventionellen Inzisionstechnik, Komma-Inzision bis zur Flapless-Inzision entwickelte. Im Vergleich sind die letzteren Techniken auf Hinsicht der Weich- wie auch Hartgewebsschonung besser bewertet worden als die konventionelle Inzision (Nageshwar 2002; Kim et al. 2011). Es wurden weniger Schmerz, Schwellung, Trismus und periodontaler Attachmentverlust belegt. Ebenfalls wurden durch eine gering dimensionierte Lappenbildung oder sogar einen 
Verzicht auf den Mukoperiostlappen, Osteoklastenaktivitäten verhindert, was zu geringeren Knochenresorptionen führte (Fickl et al. 2011).

Auch in der Technik der Osteotomie wurden gewebeschonende Methoden angestrebt. Die Bemühungen, Alternativen zur konventionellen Technik zu entwickeln, um einen großflächigen Knochendefekt zu vermeiden, wurden deutlicher. In verschiedenen Studien wurden neue Osteotomietechniken beschrieben (Pogrel et al. 2004; Tolstunov et al. 2011).

Die okklusale Expositionstechnik, die in dieser Studie vorgestellt wurde, ist prinzipiell eine Kombination aus verschiedenen Techniken mit dem Ziel einer "minimal-invasiven Operation". Die Inzision erfolgte ohne großflächige Lappenbildung, um die Vorteile wie z. B. Reduktion des Weichgewebstraumas und geringere Narbenbildung etc., die in der Studie von Kim et al. (2011) beschrieben und belegt worden sind, zu erhalten.

Die Hartgewebspräparation wurde ebenfalls minimal-invasiv gehalten, indem nur der okklusale Teil der Krone dargestellt worden ist. Anders als bei den früheren Osteotomietechniken entsteht somit nur ein geringfügiger Knochendefekt. Durch die aufeinanderfolgenden Trennungen des Zahnes ist weiterhin eine großflächige Osteotomie nicht erforderlich und mithilfe der visuellen Hilfsmittel (Endoskop und Mikroskop) konnten einzelne Fragmente, die in der Tiefe verblieben sind, problemlos entfernt werden. Zusammenfassend kann auf diese Weise eine minimal-invasive Zahnentfernung erfolgen.

Im Gegesatz zu der Koronektomie (Pogrel et al. 2004) oder perikoronalen Ostektomie (Tolstunov et al. 2011) wurde angestrebt, eine zweiphasige Behandlung $\mathrm{zu}$ vermeiden. Diesbezüglich ist eine gezielte Eruptionsförderung durch Belassung eines Zahnfragments in situ nicht erwünscht.

Die in der Studie untersuchte okklusale Expositionstechnik setzt Kenntnisse in der Anwendung von visuellen Hilfsmitteln (Endoskop und Mikroskop) voraus. Die Handhabung der visuellen Hilfsmittel sollte zuerst anhand verschiedener Übungsmittel wie z. B. am Phantom und Präparat 
erlernt werden, um diese routiniert anwenden zu können.

Vom technischen Aufwand ist die Mini-Flap-Inzision wie auch die Kronenexposition nicht schwieriger als die konventionelle Technik.

Während der raumschaffenden Trepanation sollte darauf geachtet werden, möglichst nicht mit einer Fräse die linguale Knochenwand stark zu manipulieren. Aufgrund des Nervus lingualis, der häufig auf dem Periost der lingualen Knochenwand liegt (Kiesselbach und Chamberlain 1984), sollte um eine Nervenläsion zu vermeiden ein diamantierter Kugelbohrer bevorzugt werden. Auch einer Traumatisierung des Mundbodens wird durch die weichgewebsschonende Präparation vorgebeugt. Alternativ zum diamantierten Bohrer können auch analog zur Schädelbasischirurgie, ultraschallbasierte Piezochirurgie-Geräte für einen gezielten Abtrag des Knochens angewendet werden (Schipper 2011). Diese haben den Vorteil Weichteiltraumatisierungen vorzubeugen, wobei als Nachteil eine relativ lange Anwendungszeit genannt wird (Schipper 2011).

Eine gezielte Anwendung des Endoskops oder auch Mikroskops wird dabei als visuelle Hilfe dienen.

Die einzelnen Kronenfragmente können mit der Implosionstechnik (Luxation in den Innenraum des Zahnes) getrennt entfernt werden. Dies ist auch zum Vorteil des Alveolarknochens, da keine Knochenfragmente durch eine Luxation in die Peripherie frakturiert werden können. Bei großen Fragmenten können diese durch eine weitere Trennung zerkleinert werden und somit eine Entfernung erleichtern.

Die Phase der Wurzelentfernung kann aufgrund von internen Blutungen sowie der Kavitätentiefe im Knochen erschwert sein und ist in den meisten Osteotomietechniken als anspruchsvoll zu bewerten. Um ein Wurzelfragment zu entfernen, wurde bisher die Übersicht mittels großflächiger Osteotomie erzielt. Dies ist bei der okklusalen Expositionstechnik nicht erforderlich, da eine Übersicht durch das Mikroskop und Endoskop gewährleistet wird. Auch eine gezielte mikrochirurgische Entfernung wie auch das Abtragen des Wurzelrestes ist somit möglich, was bei ankylosierten Wurzeln und Wurzeln, die in enger Beziehung mit dem Nervus alveolaris inferior liegen, von Vorteil sein kann. Hierbei wird der 
Abtrag des Knochens unmittelbar an der Wurzeloberfläche mit Hilfe diamantierter Kugelbohrer oder piezochirurgisch erzielt.

Ein weiterer Vorteil dieser Technik ist die Behandlungsposition. Während der Operateur bei der konventionellen Vorgehensweise sich seitlich des Patienten befindet, wird bei der okklusalen Expositionstechnik die 12-Uhr Position gewählt. Dadurch ist zu beiden Operationsregionen eine gute Sicht und Handhabung der Instrumente gewährleistet.

Verschiedene Aspekte können zur Bewertung einer neuen Technik in Frage kommen, wobei die Durchführbarkeit den wichtigsten Aspekt stellt. Die okklusale Expositionstechnik ist für Operateure, die bei Extraktionen gelegentlich auch die Zähne trennen, keine neue Herausforderung. Dabei ist das Risiko einer Nervenläsion des Nervus lingualis bei der okklusalen Exposition geringer, was auf die gezielte Trennung wie auch schonende Präparationsinstrumente zurückzuführen ist. Weiterhin ist eine gute Übersicht ein wichtiger Faktor hinsichtlich der Vermeidung einer Nervenläsion. Eine professionelle Handhabung mit den visuellen Hilfsmitteln ist unumgänglich, damit während der gesamten Operation eine gute Übersicht gewährleistet werden kann.

\subsubsection{Stellenwert von Mikroskop und Endoskop}

Das Endoskop und Mikroskop sind im Gebiet der Oralchirurgie oft verwendete Geräte, die zur Übersicht und Darstellung sowie einer mikrochirurgischen Operation dienen (Engelke und Capobianco 2005; Juodzbalys et al. 2008; Cortellini und Tonetti 2011). Aufgrund der minimalinvasiven Inzision wie auch Präparation, war in diesem Fall eine vollständige Übersicht des Operationsfeldes nur schwierig durchzusetzten. Aufgrund dessen wurde in dieser Studie als visuelle Unterstützung das Endoskop wie auch das Mikroskop verwendet.

In der Studie von Taschieri et al. (2008) wurde belegt, dass in der 
Durchführung einer Wurzelspitzenresektion ein Endoskop mehr Vorteile zeigt als ein Mikroskop. Das Endoskop ist hinsichtlich der Verwendungsmöglichkeiten vielseitiger und hat den Vorteil, kein fixiertes Blickfeld zu haben. Dieses bedeutet, dass das zu behandelnde Operationsfeld aus jedem Winkel und jeder Entfernung inspizierbar ist, wobei eine ständige Fokussierung und Tiefenwahrnehmung durch die Stützfunktion des Endoskops erhalten bleibt (Bahcall et al. 1999).

Im Vergleich dazu hat das Mikroskop eine starre Fixierung und das Blickfeld ist eingeschränkt, wobei besonders im hinteren Teil des Kieferabschnittes eine Bildgebung oft nicht möglich ist. Auch die Fokussierung geht verloren, sobald sich der Patient oder das Mikroskop bei einer höheren Vergrößerung bewegt, was bei einer Operation unter Lokalanästhesie nicht ausgeschlossen werden kann.

Die Bedienung dieser Geräte weist auch Vor- und Nachteile auf. Das Endoskop wird mit einer Hand gehalten und die Fokussierung wie auch der Zoom werden mit einem Finger reguliert. Der Nachteil ist dabei, dass für die Operation eine Hand blockiert ist und so Schwierigkeiten auftreten können. Beim Mikroskop ist es umständlicher mit zwei Händen das Gerät zum Zwecke der Fokussierung und Vergrößerung zu regulieren. Von Vorteil ist dabei jedoch, dass nach der richtigen Einstellung beide Hände zur Operation verwendet werden können.

Ein weiterer Nachteil bei den Endoskopen tritt bei der Reinigung auf. Bei einer Kontamination mit Gewebeflüssigkeit wie Blut und Speichel muss die Optik des Endoskops während der Anwendung mittels Spülflüssigkeit gereinigt werden. Dieser Vorgang wird bei starken Blutungen öfter benötigt, was einen verlängerten Zeitaufwand von insgesamt 4-5 min in Anspruch nimmt (Taschieri et al. 2008). Im Gegensatz dazu wird bei dem Mikroskop aufgrund des Abstands zum Operationsfeld eine Reinigung der Optik seltener durchgeführt. Dennoch ist in Bereichen, wo eine Behandlung über den Spiegel erfolgen muss, eine wiederholte Reinigung des Spiegels notwendig. 
Während der Durchführung der Studie wurden verschiedene Anwendungsbereiche der visuellen Hilfsmittel in den einzelnen Phasen beobachtet.

In der Phase der raumschaffenden Trepanation überwog die Anwendung vom Mikroskop mit 44\%, gefolgt vom Endoskop mit 22\% und die Kombination Mikroskop/Endoskop mit 18\%. In 16\% der Fälle konnte die Phase der Trepanation nicht ausgewertet werden, da hier die Lupenbrille oder keine Hilfsmittel angewendet wurden. In der weiteren Phase der Odontosektion überwog ebenfalls die Anwendung vom Endoskop mit 46\%, gefolgt vom Mikroskop mit 38\%. Die Kombination Mikroskop/Endoskop wie auch die nicht auswertbaren Fälle betrugen jeweils 8\%. In der Phase der Wurzelentfernung zeigte sich ein ähnliches Bild, wobei weiterhin die Anwendung des Endoskops mit 40\% überwog, gefolgt vom Mikroskop mit $38 \%$. Die Kombinationsanwendung blieb mit $8 \%$ gleich und $14 \%$ konnten nicht ausgewertet werden. In der letzten Phase der Alveolenmessung wurde mit 56\% die Anwendung des Endoskop bevorzugt, gefolgt vom Mikroskop 18\%. Bei 26\% konnten die Vermessung nicht ausgewertet werden.

In verschiedenen Studien wurden beide Geräte hinsichtlich der Vorteile während einer Operation untersucht (Rubinstein und Kim 2002; GarciaCalderon et al. 2007), wobei kein signifikanter Unterschied im Vergleich beider Geräte festgestellt werden konnte. Es konnte lediglich belegt werden, dass die visuelle Unterstützung im Vergleich zur Operation ohne visuelle Unterstützung positiver bewertet wurde (von Arx et al. 2003; Kontakiotis et al. 2010).

In der Funktion wie auch Anwendung von visuellen Hilfsmitteln können Vorund Nachteile entstehen, wobei der Erfolg einer Operation nicht direkt mit der Auswahl von Endoskop oder Mikroskop zusammenhängt. Letztendlich ist eine optimale intraoperative Visualisierung notwendig, um den Erfolg einer Operation auf hohem Niveau zu ermöglichen. 


\subsection{Klinische Bewertung}

\subsubsection{Operationsdauer}

Die Operationsdauer wurde zur Untersuchung wie auch Bewertung des Schwierigkeitsgrades und Risikobestimmung der postoperativen Komplikationsrate herangezogen (Valmaseda-Castellon et al. 2000). Da sich die Schwierigkeit der Operation laut der Studie von Renton et al. (2001) in einer verlängerten Operationszeit widerspiegelt, ist diese auch ein wichtiger Parameter, um eine neue Methode zu bewerten.

Einige Faktoren sind als Risikoindikatoren zu betrachten, die eine Operationszeit verlängern können. Diese sind z. B. Angulationstyp, Impaktierungsgrad, eine enge Beziehung zum Nervus alveolaris inferior und die Erfahrung des Operateurs (Benediktsdottir et al. 2004; Baqain et al. 2008). Dementsprechend wurden in dieser Studie einige Parameter berücksichtigt, um die Bewertung der Operationszeit durchzuführen.

In unserer Studie wurde eine durchschnittliche Operationszeit von 18,41 min gemessen. Diese wurde ab der raumschaffenden Trepanationsphase bis zu Alveolenausmessung bestimmt. Da aufgrund der Variationen beim Impaktierungsgrad unterschiedliche Voraussetzungen bestanden, wurde die Vorbereitungsphase (Inzision, Osteotomie) bei der Zeitbestimmung nicht berücksichtigt. Außerdem entstand durch minimalinvasive Maßnahmen (Mini-Flap-Inzision) kein wesentlicher Zeitbedarf bei der Lappenbildung und der späteren Nahtversorgung.

Im Vergleich zur konventionellen Technik, mit Durchschnittszeiten von 14,62 min (Renton et al. 2001), 22,63 min (Bello et al. 2011) und $38 \mathrm{~min}$ (Chey et al. 1993), wurde kein erhöhter Zeitbedarf festgestellt.

Die linguale Split-Technik wurde hinsichtlich der Operationszeit untersucht, wobei eine Variation von 17,7 min (Praveen et al. 2007) bis zu 36,3 min (Hindy et al. 1995) belegt wurde. Somit ist auch im Vergleich zu anderen Techniken die Operationszeit im realistisch durchführbaren Bereich.

Es sollte auch beachtet werden, dass in unserer Studie überwiegend Zähne mit unmittelbarem Nervenkontakt behandelt wurden (92\%). Im 
Vergleich zur Arbeit von Kim et al. (2011), in der nur teilretinierte Zähne operiert worden sind, ist somit ein höherer Zeitaufwand durch den Impaktierungsgrad und Schwierigkeitsgrad in unserer Arbeit unvermeidbar.

Würde man die Zähne nach Einteilung der Angulationstypen betrachten, gibt es bei den vier Angulationstypen keinen signifikanten Unterschied. Das bedeutet, dass alle Angulationstypen mit der okklusalen Expositionstechnik einen relativ ähnlichen Zeitaufwand in Anspruch nehmen.

In der Studie konnte lediglich eine Tendenz ermittelt werden, die über Durchschnittszeiten der Operation erfolgen konnte. Diese deutet darauf hin, dass die vertikal (22,58 min) angulierten Zähne am zeitaufwändigsten waren, wobei für diesen Angulationstyp ein herausstechender Wert $(89,5$ min) ermittelt wurde. Würde dieser Extrem-Wert nicht miteinbezogen werden, wäre die Reihenfolge der Operationszeit folgende: horizontal (21,55 min) > mesioangulär $(16,11 \mathrm{~min})>$ vertikal $(15,9 \mathrm{~min})>$ distoangulär (12,1 min). Da aber in unserer Studie alle Werte mit ins Ergebnis einbezogen worden sind, folgen den vertikal angulierten Weisheitszähnen in absteigender Reihenfolge die horizontal, mesioangulären und distoangulären Zähne.

Bei der Operation der vertikal angulierten Zähne war aufgrund der Knochentiefe, besonders bei impaktierten Zähnen, die Entfernung der Wurzelreste schwierig. Dabei ergab sich beim Schweregrad der Operation ein Unterschied zu anderen Studien. Einige Studien belegen, dass die horizontal angulierten Zähne aufgrund der längsten Operationstzeit am schwierigsten zu entfernen waren (Benediktsdottir et al. 2004; Baqain et al. 2008). Anderseits belegen andere Studien, dass distoanguläre und mesioanguläre Weisheitszähne am schwierigsten zu entfernen waren, was mit intensiverem Knochenabtrag und Platzmangel bei der Instrumentenanwendung begründet wurde (Blondeau und Daniel 2007; Malkawi et al. 2011).

Hinsichtlich der Phasen ergab sich bei relativ eng beieinander liegenden 
Durchschnittszeiten (Phase 1: 5,76 min, Phase 2: 6,67 min, Phase 3: 5,87 min, Phase 4: $52 \mathrm{~s}$ ) die höchste Streuung bei der dritten Phase (40 s bis $53 \mathrm{~min}$ ). Dieses deutet daraufhin, dass in der Wurzelentfernungsphase, je nach Situation der Wurzellage, die meiste Variation bei der Entfernung entstand. Die zweite Phase folgt mit einer Streuung von $20 \mathrm{~s}$ bis 30,78 min und daraufhin die erste Phase mit einer Streuung von $42 \mathrm{~s}$ bis $15 \mathrm{~min}$. Die niedrigste Streuung ergab sich in der vierten Phase (18 $\mathrm{s}$ bis $101 \mathrm{~s}$ ), was sich durch ein routiniertes Messverfahren erklären lässt.

Aufgrund der verschiedenen Angulationstypen wie auch Krümmungsmuster und Anzahl der Wurzeln könnte solch eine Variation möglich sein.

\subsubsection{Knochenverlust}

Bei einer operativen Weisheitszahnentfernung wird immer mit einem gewissen Knochenverlust gerechnet. Dies ist die Voraussetzung, um einen impaktierten oder teilretinierten Weisheitszahn sicher zu entfernen ohne weitere Traumatisierungen des Nachbargewebes zu verursachen.

Bei der konventionellen Osteotomie wird empfohlen, soviel vom Alveolarknochen abzutragen bis die Schmelz-Zement-Grenze sichtbar wird (Klammt 2000), um eine sichere Retention zu erzielen und den Zahn mit dem Hebel bzw. mit der Zange fassen zu können. Durch den häufigen vestibulären Knochenabtrag wird die bukkale Knochenwand geschwächt. Diese Schwächung wird durch den Knochendefekt der leeren Alveole zusätzlich verstärkt. Dies ist auch in geringen Fällen eine Ursache postoperativer Kieferfrakturen (Schneider und Stern 1971).

Auch die spätere Regeneration des Knochens nach einer Zahnextraktion wird durch einen Knochenverlust gekennzeichnet, wobei zwei Drittel der ursprünglichen Knochenhöhe wieder erreicht werden können (Amler 1969). Durch vorangegangene Studien wurde belegt, dass die bukkale Alveolenwand im Vergleich zur oralen Wand einen höheren Knochenverlust post extraktionem aufweist (Pietrokovski und Massler 1967; Araujo und Lindhe 2005). Dies ist nicht nur im Molarenbereich, sondern auch im Frontzahnbereich nachgewiesen worden. Im Frontzahnbereich stellt dieser 
Knochenverlust in der Implantologie oftmals ein erhebliches ästhetisches Problem dar (Bidra und Chapokas 2011).

Um einen Knochendefekt und somit auch die nachfolgende Knochenresorption zu reduzieren, wurde in unserer Studie versucht, den Defekt der bukkalen Alveolenwand möglichst gering zu halten.

In verschiedenen Studien wurde durch modifizierte Inzisionen versucht, die physiologische Knochenresorption nach einem Defekt gering zu halten (Nageshwar 2002, Fickl et al. 2011). Obwohl diese Hypothese in verschiedenen Studien kontrovers diskutiert wird, ist eine Knochenresorption nach einer Inzision ohne Lappenbildung mit positiven Ergebnissen belegt worden (Kim et al. 2011). Dieses wurde auch in der vorliegenden Untersuchung berücksichtigt, weswegen wir eine Inzisionsmethode ohne großflächige Lappenbildung (Mini-Flap-Inzision) gewählt haben. Zusätzlich wurde in der Phase der Osteotomie zur Reduktion des Knochendefektes die bukkale Knochenwand nicht präpariert, sondern möglichst nur krestal bearbeitet, um so einen okklusalen Zugang zu schaffen.

Die Ergebnisse der prä- und postoperativ gemessen Werte zeigten einen Knochenverlust von durchschnittlich $2 \mathrm{~mm}(0,4 \mathrm{~mm}$ bis zu 6,5 mm). Dies ist im Vergleich zur konventionellen Technik, die bukkal bis zur SchmelzZement-Grenze osteotomiert wird, welches fast die Hälfte der Zahnlänge oder mehr ausmacht, ein erheblicher Gewinn. Es sollte zusätzlich beachtet werden, dass eine Standardisierung der Parameter durchgeführt wurde. Die Fehlerfaktoren bezüglich des Vergleichs Röntgenbild-intraorale Messung wurden darauf hin mit einem ausgerechneten Fehlerfaktor (Maximalwert von $7 \%$ ) bei jedem postoperativ gemessenen Wert abgezogen. Das bedeutet aufgrund der Tatsache, dass nicht bei jedem Zahn realistisch ein Messfehler von maximal 7\% entsteht, dass mit einem Knochenverlust von weniger als $2 \mathrm{~mm}$ zu rechnen ist.

Weiterhin wurde versucht einen Unterschied des Knochenverlustes in Relation zu den verschiedenen Angulationstypen zu ermitteln. Das 
Ergebnis zeigte, dass kein signifikanter Unterschied zwischen allen vier Angulationstypen besteht.

Es zeigte sich ebenfalls ein relativ konstanter Knochenverlust von 2-3 mm, was bedeutet, dass mit dieser Technik bei den verschiedenen Angulationstypen kein Unterschied hinsichtlich des Knochenverlustes besteht.

\subsubsection{Komplikationen}

\subsubsection{Intraoperative Komplikationen}

Intraoperative Komplikationen sind unerwünschte Operationsereignisse, die im Zusammenhang mit dem Allgemeinbefinden des Patienten stehen oder durch den Operateur ausgelöst werden können. Als intraoperative Komplikationen zählen Blutungen, Schädigung des Nachbarzahnes, Traumatisierung des Nachbargewebes, Anästhesie-bedingte Schädigung und Nervenläsion durch den Wundhaken (Van Gool et al. 1977; Arrigoni und Lambrecht 2004). Diese können auch zu weiteren postoperativen Komplikationen führen.

In den verschiedenen Operationstechniken der Weisheitszahnentfernung wurde angestrebt, die Komplikation möglichst gering zu halten.

Bei der lingualen Split-Technik wurde erwähnt, dass es aufgrund der massiven Blutung beim Ablösen der lingualen Knochenplatte vom Periost zu Modifikationen kam.

Auch bei der Koronektomie (Ecuyer und Debien 1984; Pogrel et al. 2004) wurde anfangs der Wurzelanteil belassen, welcher durch häufigeres Auftreten von Komplikationen später entfernt werden musste. Eine weiterführende Methode der perikoronalen Ostektomie (Tolstunov et al. 2011) ermöglichte durch eine zweizeitige Operation, kein Belassen von Wurzelresten und somit die Komplikationsrate zu senken. Allerdings wurde die zweizeitige Operation von den Patienten als Belastung empfunden. 
Die okklusale Expositionstechnik ist aufgrund der minimal-invasiven Operationstechnik der Weich- wie auch Hartgewebspräparation hinsichtlich der intraoperativen Komplikationen schonender als die herkömmlichen Techniken. Dieses spiegelte sich in den Ergebnissen wieder, da es intraoperativ nicht zu Komplikationen kam. Es ist somit eine relativ atraumatische Operation möglich.

\subsubsection{Postoperative Komplikationen}

Verglichen mit den intraoperativen Komplikationen sind die postoperativen Komplikationen zahlreicher (Arrigoni und Lambrecht 2004).

Im Vergleich zum Oberkiefer sind die postoperativen Komplikationen im Unterkiefer häufiger zu beobachten (Reichart 1995), da dieser eine exponierte Stellung für die Retention von Speiseresten, Zerfallsprodukten und Speichel aufweist. Auch die anatomische Gegebenheit der kompakteren Knochenstruktur mit geringerer Vaskularisierung kann ein Grund dafür sein (Krüger 1989; Schroeder 1997).

Wundheilungsstörungen, wie Infektionen und/oder Entzündungen (Alveolitis, Osteomyelitis), Sensibilitätsstörungen, Kieferbrüche, Belassen eines Wurzelfragmentes und Nachblutungen sind die am häufigsten postoperativ diagnostizierten Komplikationen, die belegt worden sind (Mercier und Precious 1992; Barbosa-Rebellato et al. 2011).

Bei der okklusalen Expositiontechnik sollte die postoperative Komplikationsrate so gering wie möglich gehalten werden, indem eine minimal-invasive Operation angewendet wird. Die Gefahr einer Infektion sollte anhand einer kleinflächigen Wunde vermieden werden. Da in der Operation kein Wundhaken zum Abhalten der lingualen Schleimhaut sowie zum Schutz des Nervus lingualis verwendet wurde, sollte eine Traumatisierung des Nerven durch das Instrumentarium ebenfalls vermieden werden. Ein weiterer Schutz waren auch die diamantierten Kugelbohrer oder alternativ die Piezochirurgie-Geräte, die einer Nervenläsion besser vorbeugen als Fräsen und Rosenbohrer. Alternativ 
zum Schutz des Nervus lingualis, war in dieser Technik das gezielte Abtragen der okklusalen Knochenoberfläche, wobei die bukkale sowie die linguale Knochenwand nicht osteotomiert wurden. Ebenfalls ist auch ein geringeres Risiko eines Kieferbruches durch diesen minimalen Knochenabtrag zu erzielen. Die Anwendung von visuellen Hilfsmitteln ermöglicht des Weiteren auch bei engem Kontakt zum Nervus alveolaris inferior einen Überblick in die tiefe Region, was zu einem geringeren Risiko einer Nervenläsionsentstehung beitragen kann.

In dieser Studie wurde insgesamt in sechs Fällen (12\%) eine postoperative Komplikation festgestellt. In drei Fällen (6\%) waren Sensibilitätsstörungen des Nervus alveolaris inferior aufgetreten, die aber innerhalb von zwei bis drei Monaten zurückgingen. Hierbei muss erwähnt werden, dass die aufgetretene Komplikation als gering betrachtet werden kann, da 92\% der hier untersuchten Weisheitszähne eine enge Beziehung zum Nervus alveolaris inferior aufwiesen. Dieser Aspekt wird in vielen Studien als bedeutender Risikofaktor bewertet (Gülicher und Gerlach 2001; Bui et al. 2003).

Hinsichtlich der Sensibilitätsstörung ist während dieser Studie keine Komplikation im Zusammenhang mit dem Nervus lingulis aufgetreten, was als positiver Effekt der okklusalen Expositionstechnik betrachtet werden kann.

Weiterhin wurden in zwei Fällen (4\%) putride Entzündungen festgestellt, wobei eine genaue Ursache nicht zu finden war. In beiden Fällen war der Patient weiblich und die Zähne hatten eine mesial angulierte Position. Nach Bui et al. (2003) und Blondeau und Daniel (2007) stellten das Geschlecht sowie der Angulationstyp Risikofaktoren dar.

Im ersten Fall könnten das Alter (>25) (Chiapasco et al. 1995; Chuang et al. 2007; Barbosa-Rebellato et al. 2011) sowie die Operationszeit als Risikofaktoren betrachtet werden (Renton et al. 2001; Lago-Mendez et al. 2007), wobei die Operationszeit auch kontrovers diskutiert wird (Benediktsdottir et al. 2004). Im zweiten Fall war eine Spätinfektion aufgetreten, die sich erst nach zwei Monaten durch eine Abzessbildung äußerte. Das Alter sowie 
auch die Operationszeit konnten hierbei nicht als Risikofaktoren betrachtet werden. Ob es sich an dieser Stelle um eine chronische Entzündung, Spätinfektion, Fremdkörperreaktion auf Bohrspanrückstände, eingedrungene Speisereste oder schlechte Mundhygiene handelte, konnte nach Aussage von Filippi (2001) nicht sicher belegt werden.

Einmalig (2\%) war auch ein Wurzelfragment verblieben, welches durch einen revidierenden Eingriff entfernt werden konnte.

Um einen Zusammenhang zwischen der Zahnposition und der aufgetretenen Komplikation zu erhalten, wurden diese Parameter überprüft. Ein signifikanter Unterschied konnte dabei nicht festgestellt werden.

Im Gegensatz zu unserer Untersuchung konnte in der Studie von Malkawi et al. (2011) die höchste Komplikationsrate bei den distoangulären Weisheitszähnen belegt werden und bei Bui et al. (2003) bei den mesioangulären Weisheitszähnen.

Weiterhin wurde kein signifikanter Zusammenhang zwischen der Operationszeit und der Komplikationsrate festgestellt. 


\section{Zusammenfassung}

Hintergrund: Die Osteotomie von Weisheitszähnen ist im zahnärztlichen Alltag ein Routineverfahren. Das primäre Ziel der modernen Operationsverfahren ist die Reduktion operationsbedingter Morbidität und der Erhalt von umgebendem Knochen.

Material \& Methode: Bei 40 Patienten (19 Männer, 21 Frauen), im Alter von 15-55 (27,27) Jahren, wurden 50 Unterkieferweisheitszähne uni- oder bilateral mittels der okklusalen Expositionstechnik entfernt. 92\% der Zähne wiesen einen engen Kontakt ( $\leq 2 \mathrm{~mm}$ ) zum Nervus alveolaris inferior auf. Die Operationen wurden durch die visuellen Hilfsmittel Mikroskop und Endoskop unterstützt. Nach Freilegung der Krone wurde bei der okklusalen Expositionstechnik eine gezielte raumschaffende Trepanation des Zahnes durchgeführt. Dieser raumschaffende Vorgang diente zur Fragmentmobilisierung, indem die Fragmente in den Freiraum hineinluxiert wurden (Implosionstechnik). Weiterhin wurden die verbliebenen Fragmente gezielt durchtrennt und einzeln entfernt. Alle Operationsschritte wurden videodokumentiert und ausgewertet. Um den prä- und postoperativen Knochenverlust zu ermitteln, wurden Röntgenbilder vermessen und mit intraoralen Messungen verglichen. Dabei wurde die bukkale Lamelle intraoperativ endoskopisch und mikroskopisch durch Sondierung vermessen.

Ergebnisse: Von 50 Weisheitszähnen waren 15 vertikal (30\%), jeweils 14 mesial (28\%) und horizontal (28\%) und 7 distal (14\%) anguliert. Die mittlere Operationszeit betrug 18,41 $\mathrm{min}$ (4-89,5 $\mathrm{min}$ ), wobei sich zwischen den einzelnen Angulationstypen kein signifikanter Unterschied zeigte (vertikal 22,58 min, horizontal - 21,55 min, mesial - 16,11 min, distal - 12,10 min). Das Operationsfeld wurde durchgängig mittels Endoskop und Mikroskop beobachtet, was anhand der visuellen Dokumentationen festzustellen war (Phase 1: Mikroskop 44\%, Endoskop 22\%, Mikroskop \& Endoskop 18\%, Sonstiges 16\%; Phase 2: Mikroskop 38\%, Endoskop 46\%, Mikroskop \& 
Endoskop 8\%, Sonstiges 8\%; Phase 3: Mikroskop 38\%, Endoskop 40\%, Mikroskop \& Endoskop 8\% Sonstiges 14\%; Phase 4: Mikroskop 18\%, Endoskop 56\%, Sonstiges 26\%). Hier wurde mit zunehmender Tiefe vermehrt das Endoskop eingesetzt.

Der durchschnittliche Knochenverlust betrug ca. 2 mm (0,4-6,5 mm), wobei eine gleichmäßige Verteilung in allen vier Angulationstypen zu ermitteln war. Intraoperative Komplikationen traten nicht auf $(0 \%)$. In sechs Fällen (12\%) wurden postoperative Komplikationen beobachtet, drei temporäre Hypoder Parästhesien (6\%), zwei Entzündungen (4\%) und ein verbliebener Wurzelrest (2\%).

Schlussfolgerung: Die okklusale Expositionstechnik mit endoskopischer und mikroskopischer Visualisierung erlaubt unter Verzicht einer großflächigen Lappenbildung eine gezielte und schonende Zahnseparation. Das gilt insbesondere für die Entfernung komplex verlagerter Zähne mit enger Lagebeziehung zum Nervus alveolaris inferior. Die okklusale Expositions-technik ist auch bei Zähnen mit unmittelbarem Nervenkontakt anwendbar.

Bei der Weisheitszahnentfernung und analog auch in anderen Kieferregionen kann diese Methode Knochenverluste und großflächige Gewebetraumata vermeiden.

Dem Nachteil eines erhöhten Zeitaufwandes stehen eine hohe Präzision der Op-Techniken und eine bessere Übersicht in komplexen Fällen positiv gegenüber. 


\section{Literaturverzeichnis}

Alling CC (1986):

Dysesthesia of the lingual and inferior alveolar nerves following third molar surgery. J Oral Maxillofac Surg 44, 454-457

\section{Amler MH (1969):}

The time sequence of tissue regeneration in human extraction wounds.

Oral Surg Oral Med Oral Pathol 27, 30939318

Andrade Munhoz E, Bodanezi A, Ferreira Junior O, Mauro Granjeiro J (2011): Bone crestal height and bone density after third molar extraction and grafting: a longterm follow-up study.

Clin Oral Invest $\underline{15}, 123-126$

\section{Appiah-Anane S, Appiah-Anane MG (1997):}

Protection of the lingual nerve during operations on the mandibular third molar: a simple method.

Br J Oral Maxillofac Surg $\underline{35}, 170-172$

Araujo MG, Lindhe J (2005):

Dimensional ridge alterations following tooth extraction. An experimental study in the dog.

J Clin Periodontol $\underline{32}, 212-218$

Arrigoni J, Lambrecht JT (2004):

Komplikationen bei und nach operativer Weisheitszahnentfernung.

Schweiz Monatsschr Zahnmed 114, 1271-1279

\section{Azaz B, Taicher S (1982):}

Indications for the removal of the mandibular impacted third molar.

J Can Dent Assoc 4ㅇ, 731-734

\section{Bähr W (1996):}

Die operative Entfernung der unteren Weisheitszähne.

Zahnärztl Mitt $\underline{\text { 86, 886-892 }}$

\section{Bahcall JK, Di Fiore PM, Poulakidas TK (1999):}

An endoskopic technique for endodontic surgery.

J Endod 25, 132-135 
Baqain ZH, Karaky AA, Sawair F, Khaisat A, Duaibis R, Rajab LD (2008):

Frequency estimates and risk factors for postoperative morbidity after third molar removal: a prospective cohort study.

J Oral Maxillofac Surg 66, 2276-2283

Barbosa-Rebellato NL, Thomé AC, Costa-Maciel C, Oliveira J, Scariot R (2011): Factors associated with complications of removal of third molars: a transversal study. Med Oral Patol Oral Cir Bucal 16, 376-380

\section{Bell GW (2004):}

Use of dental panoramic tomographs to predict the relation between mandibular third molar teeth and the inferior alveolar nerve. Radiological and surgical findings, and clinical outcome.

$\mathrm{Br} \mathrm{J}$ Oral Maxillofac Surg 느, 21-27

Bello SA, Adeyemo WL, Bamgbose BO, Obi EV, Adeyinka AA (2011):

Effect of age, impaction types and operative time on inflammatory tissue reactions following lower third molar surgery.

Head Face Med 28, 1-8

Benediktsdottir IS, Wenzel A, Petersen JK, Hintze H (2004):

Mandibular third molar removal: risk indicators for extended operation time, postoperative pain, and complcations.

Oral Surg Oral Med Oral Pathol Oral Radiol Endod 97, 438-446

Bidra AS, Chapokas AR (2011)

Treatment planning challenges in the maxillary anterior region consequent to severe loss of buccal bone.

J Esthet Restor Dent 23, 354-361

Birn H (1966):

The vascular supply of the periodontal membrane. An investigation of the number and size of perforation in the alveolar wall.

J Periodontal Res $\underline{1}, 51-68$

Blackburn CW, Bramley PA (1989):

Lingual nerv damage associated with the removal of lower third molar.

Br Dent J $\underline{167}, 103-107$

Blondeau F, Daniel NG (2007):

Extraction of the impacted mandibular third molars: postoperative complications and their risk faktor.

J Can Dent Assoc 그, 325a-325e 


\section{Brägger U, Pasquali L, Kornman KS (1988):}

Remodelling of interdental alveolar bone after periodontal flap procedures assessed by means of computer-assisted densitometric image analysis (CADIA).

J Clin Periodontol $\underline{15}, 558-564$

Bruce RA, Frederickson GC, Small GS (1980):

Age of patients and morbidity associated with mandibular third molar surgery .

J Am Dent Assoc 101, 240-245

Bui CH, Seldin EB, Dodson TB (2003):

Types, frequencues, and risk factors for complications after third molar extraction.

J Oral Maxillofac Surg $\underline{61}, 1379-1389$

Caplanis N, Lozada JL, Kan JY (2005):

Extraction Defect: assesment, classification and management.

J Calif Dent Assoc 33, 853-863

Carmichael FA, McGowan DA (1992):

Incidence of nerve damage following third molar removal: a West of Scotland Oral Surgery Research Group study.

$\mathrm{Br} \mathrm{J}$ Oral Maxillofac Surg $\underline{30}, 78-82$

Chey EP, Young IG, Osborn GA, Rudkin GE (1993):

Outcomes after same day oral surgery: a review of 1180 cases at a major teaching hospital.

J Oral Maxillofac Surg $\underline{51}, 846-849$

Chiapasco M, Lorenzo DC, Marrone G (1993):

Side effects and complications associated with third molar surgery.

Oral Surg Oral Med Oral Pathol $\underline{76}, 412-420$

Chiapasco M, Crescentini M, Romanoni G (1995):

Germectomy or delayed removal of mandibular impacted third molar: the relationship between age and incidence of complications.

J Oral Maxillofac Surg $\underline{53}, 418-422$

\section{Choi B:}

Flapless implantology.

Quintessence, London 2010.

Choi B, Engelke W (2009):

Flapless implantology-Möglichkeiten und Grenzen.

Implantologie 17, 139-152 
Chuang SK, Perrott DH, Susarla SM, Dodson TB (2007):

Age as a risk factor for third molar surgery complikations.

J Oral Maxillofac Surg 타, $1685-1692$

\section{Cortellini P, Tonetti MS (2011):}

Clinical and radiographic outcomes of the modified minimally invasive surgical technique with and without regenerative materials: a randomized-controlles trial in intra-bony defects.

J Clin Peridontol $\underline{38}, 365-373$

Davis WH, Hochwald DA, Kaminishi RM (1983):

Modified distolingual splitting technique for removal of impacted mandibular third molars: technique.

Oral Surg Oral Med Oral Pathol $\underline{56}, 2-8$

\section{Drews U:}

Taschenatlas Embryologie, 2.Auflage;

Thieme Verlag, Stuttgart 2006

Dunstan SP, Sugar AW (1997):

Fractures after removal of wisdom teeth.

Br J Oral Maxillofac Surg $\underline{35}, 396-397$

Ecuyer J, Debien J (1984):

Surgical deductions.

Actual Odontostomatol $\underline{38}, 695-702$

Engelke WG (2002):

In situ examination of implant sites with support immersion endoscopy.

Int J Oral Maxillofac Implants 17, 703-706

\section{Engelke W, Capobianco M (2005):}

Flapless sinus floor augmentation using endoscopy combined with CT-scan designed surgical templates: method and report of 6 consecutive cases.

Int J Oral Maxillofac Implants 므, 891-897

\section{Engelke W, Bierbaum J, Choi EJ (2011):}

Die mikrochirurgische Entfernung unterer dritter Molaren über einen okklusalen Zugang.

Dtsch Zahnärztl Z $\underline{66}, 415-423$ 
Fickl S, Kebschull M, Schupbach P, Zuhr O, Schlagenhauf U, Hürzeler MB (2011):

Bone loss after full-thickness and partial-thickness flap elevation.

J Clin Periodontol $\underline{38}, 157-162$

Filippi A (2001):

Wundheilung und Heilungsstörungen nach Entfernung dritter Molaren.

Schweiz Monatsschr Zahnmed 111, 847-856

Garcia-Calderon M, Torres-Lagares D, Calles-Vazques C, Uson-Gargallo J, Gutierrez-Perez JL (2007):

The application of microscopic surgery in dentistry.

Med Oral Patol Oral Cir Bucal 12, 311-316

Goetzke HG, Klammt J (1991):

Die Größe der Wundfläche und die Häufigkeit der Alveolitis nach Zahnextraktion.

Dtsch Z Mund Kiefer Gesichtschir 15, 306-310

Gold SI, Hasselgren G (1992):

Peripheral inflammatory root resorption: a review of the with case reports.

J Clin Periodontol $\underline{19}$, 523-534

Greenstein G, Tarnow D (2006):

The mental foramen and nerve: clinical and anatomical factors related to dental implant placement: a literture review.

J Periodontol $\underline{77}$, 1933-1943

Gülicher D, Gerlach KL (2001):

Sensory impairment of the lingual and inferior alveolar nerves following removal of impacted mandibular third molar.

Int J Oral Maxillofac Surg 30, 306-312

Güven O, Keskin A, Akal UK (2000):

The incidence of cysts and tumors around impacted third molars.

Int J Oral Maxillofac Surg 29, 131-135

Hellwig E, Klimek J, Attin TH:

Einführung in die Zahnerhaltung

Urban \& Fischer, München/Jena 1999 
Heurich T, Ziegler C, Steveling H, Wörtche R, Mühling J, Hassfeld S ( 2002):

Digital volume tomography - an extension to the diagnostic procedures available for application before surgical removal of third molars.

Mund Kiefer Gesichtschir 므, 427-432

Hindy AM, Ismaiel ME, Fayed N (1995):

Modified lingual split technique versus conventional buccal technique in odontectomy of impacted third molars.

Egypt Dent J $\underline{41}, 1137-1144$

\section{lasella JM, Greenwell H, Miller RL, Hill M, Drisko C, Bohra AA, Scheetz JP (2003):}

Ridge preservation with freeze-dried bone allograft and a collagen membrane compared to extraction alone for implant site development: a clinical and histologic study in humans.

J Periodontol $\underline{74}$, 990-999

Jensen OT, Cullum DR, Baer D (2009):

Marginal bone stability using 3 different flap approaches for alveolar split expansion for dental Implants:a 1-year clinical study.

J Oral Maxillofac Surg 67, 1921-1930

\section{Junqueira L, Carneiro J, Kelly R:}

Histologie. 4.Auflage;

Springer-Verlag. Berlin/Heidelberg 1996

Juodzbalys G, Bojarskas S, Kubilius R, Wang HL (2008):

Using the support immersion endoscope for socket assessment.

J Periodontol $\underline{79}, 64-71$

Kiesselbach JE, Chamberlain JG (1984):

Clinical and anatomic observation on the relationship of the lingual nerve to the mandibular third molar region.

J Oral Maxillofac Surg $\underline{42}, 565-567$

Kim HR, Choi BH, Engelke W, Serrano D, Xuan F, Mo DY (2011):

A comparative study on the extractions of partially impacted mandibular third molars with or without a buccal flap-a prospective study.

J Oral Maxillofac Surgery $\underline{69}$, 966-970

\section{Kirtiloglu T, Bulut E, Sümer M, Cengiz I (2007):}

Comparison of 2 flap design in the periodontal healing of second molar after fully imacted mandibular third molar extractions.

J Oral Maxillofac Surg 65, 2206-2210 
Klammt J (2000):

Praxis der Zahnentfernung.

Forum med-dent, 4-85

Knutsson K, Lysell L, Rohlin M, (1989):

Post-operative status after partial removal of the mandibular third molar.

Swed Dent J $\underline{13}, 15-22$

Kontakiotis EG, Palamidakis FD, Farmakis ET, Tzanetakis GN (2010):

Comparison of isthmus detection methods in the apical third of mesial roots of maxillary and mandibular first molars: macroscopic ovservation versus operating microscope.

Braz Dent J 21, 428-431

Krausz AA, Machtei EE, Peled M (2005):

Effect of lower third molar extraction on attachment level and alveolar bone height of the adjacent second molar.

Int J Oral Maxillofac Surg $\underline{34}, 756-760$

Kristen K (1978):

Retinierte Zähne.

Zahnärztl Welt 87, 167-170

\section{Krüger R:}

Operationslehre für Zahnärzte.

Quintessenz Verlags-GmBH, Berlin 1989

Kugelberg CF, Ahlströhm U, Ericson S, Hugoson A (1985):

Periodontal healing after impacted lower third molar surgery: a retrospective study. Int J Oral Surg 14, 29-40

\section{Kunkel M, Wagner W (2006):}

Leitlinie: operative Entfernung von Weisheitszähnen.

Zahnärztliche Zentralstelle Qualitätssicherung im Institut der Deutschen Zahnärzte $1-8$

Lago-Mendez L, Diniz-Freitas M, Senra-Rivera C, Gude-Sampedro F, Rey JMG, Garcia-Garcia A (2007):

Relationships between surgical difficulty and postoperative pain in lower third molar extractions.

J Oral Maxillofac Surg $\underline{65}, 979-983$ 


\section{Leesemann K:}

Zur Entfernung retinierter Weisheitszähne und präoperativen Risikoabschätzung intraoperativer Komplikationen.

Med. Diss. Köln 2001

\section{Lekovic V, Kenney EB, Weinlaender M, Han T, Klokkevold P, Nedic M, Orsini M} (1997):

A bone regenerative approach to alveolar ridge maintenance following tooth extraction. Report of 10 cases.

J Periodontol $\underline{68}, 563-570$

\section{Lopes V, Mumenya R, Feinmann C, Harris M (1995):}

Third molar surgery: an audit of the indications for surgery, postoperative complaints and patient satisfaction.

$\mathrm{Br} J$ Oral Maxillofac Surg $\underline{33}, 33-35$

\section{Malkawi Z, Al-Omiri MK, Khraisat A (2011):}

Risk indicators of postoperative complications following surgical extraction of lower third molars.

Med Princ Pract 20, 321-325

\section{McMillan HW (1924):}

The structures and functions of the alveolar process.

J Am Dent A 11,1059

\section{Mercier P, Precious D (1992):}

Risk and benefits of removal of impacted third molars. A critical review of the literature.

J Oral Maxillofac Surg 21, 17-27

\section{Merskey H, Bogduk H:}

Classification of chronic pain, second edition, Part III: Pain terms, a current list with definitions and notes on usage.

IASP Press, Seattle 1994

\section{Middlehurst RJ, Barker GR, Rood JP (1988):}

Postoperative morbidity with mandibular third molar surgery. A comparison of two techniques.

J Oral Maxillofac Surg 46, 474-475

\section{Nageshwar (2002):}

Comma incision for impacted mandibular third molar.

J Oral Maxillofac Surg 60, 1506-1509 


\section{Neumann F:}

Der Einfluss des chirurgischen Vorgehens zum Schutz des Nervus lingualis bei der Entfernung unterer Weisheitszähne - Eine prospektive Studie.

Med.Diss. Greifswald 2003

Nordenram A, Hultin M, Kjellman O, Ramström G (1987):

Indications for surgical removal of the mandibular third molar. Study of 2,630 cases.

Swed Dent J 11, 23-29

\section{Otten J:}

Odontogene Entzündungen; in Zahnärztliche Chirurgie Band 1; hrsg.v. Reichart PA, Hausamen JE, Becker J, Neukam F, Schliephake H, Schmelzeisen R

Quintessenz Verlags-GmbH Berlin 2002, 503-505

\section{Pasler FA:}

Zahnärztliche Radiologie

Thieme-Verlag, Stuttgart 1995

\section{Pederson G:}

Oral surgery.

Saunders, Philadelphia 1988

Pell GJ, Gregory BT (1933):

Impacted mandibular third molars; classification and modified techniques for removal.

Dent Dig $\underline{39}$, 330-338

\section{Pfeiffer J (1965):}

The reaction of alveolar bone to flap procedures in man.

Periodontics $\underline{3}, 135-141$

\section{Pietrokovski J, Massler M (1967):}

Alveolar ridge resorption following tooth extraction.

J Pros Dent 17, 21-27

Pogrel MA, Goldman KE (2004):

Lingual flap retraction for third molar removal.

J Oral Maxillofac Surg 62, 1125-1230

Pogrel MA, Lee JS, Muff DF (2004):

Coronectomy: a technique to protect the inferior alveolar nerv.

J Oral Maxillofac Surg $\underline{62}, 1447-1452$ 
Praveen G, Rajesh P, Neelakandan RS, Nandagopal CM (2007):

Comparison of morbidity following the removal of mandibular third molar by lingual split, surgical bur and simplified split bone technique.

Indian J Dent Res $\underline{18}, 15-18$

Regezi JA, Kerr DA, Courtney (1978):

Odontogenic tumors: analysis of 706 cases.

J Oral Surg $\underline{36}, 771-778$

Reich W, Maurer P, Schubert J (2005):

Intraoperative Komplikationen in der zahnärztlichen Chirurgie.

Mund Kiefer GesichtsChir $\underline{9}$, 369-376

Reichart PA :

Dentoalveoläre Chirurgie; in Mund-, Kiefer- und Gesichtschirurgie; hrsg. v.

Hausamen JE, Machtens E, Rheuter J

Springer, Berlin 1995

Renton T, Smeeton N, McGurk M (2001):

Factors predictive of difficulty of mandibular third molar surgery.

$\mathrm{Br}$ Dent J $\underline{190}$, 607-610

Robinson PP, Smith KG (1996):

Lingual nerv damage during lower third molar removal: a comparison of two surgical method.

Br Dent J $\underline{180}, 456-461$

Rubinstein RA, Kim S (2002):

Long-term follow-up of cases considered healed one year after apical microsurgery.

J Endod 28, 378-383

Sands T, Pynn BR, Nenniger S (1993):

Third molar surgery: current ceoncepts and controversies. Part1.

Oral Health $\underline{85}, 11-14,17$

Schersten E, Lysell L, Rohlin M (1989):

Prevalence of impacted third molars in dental students.

Swed Dent J 13, 7-13

Schilli W, Krekeler G:

Der Verlagerte Weisheitszahn.

Quintessenz Verlag - GmbH, Berlin 1984 
Schipper J (2011)

Interdisziplinäre Schädelbasischirurgie. Von der explorativen Kraterloch- zur prädiktiven Tunnelchirurgie.

HNO $\underline{59}, 327-331$

Schleier P, Bierfreund G, Schultze-Mosgau S, Moldenhauer F, Küpper H, Freilich M (2008):

Simultaneous dental implant placement and endoscope-guided internal sinus floor elevation: 2-year post-loading outcomes.

Clin Oral Implants Res $\underline{19}$, 1163-1170

Schneider S, Stern M (1971):

Teeth in the line of mandibular fractures.

J Oral Surg $\underline{29}, 107-109$

\section{Schroeder H:}

Pathobiologie oraler Strukturen - Zähne, Pulpa, Parodont.

Karger, Basel 1997

\section{Schroeder H:}

Orale Strukturbiologie: Entwicklungsgeschichte, Struktur und Funktion normaler Hart- und Weichgewebe der Mundhöhle und des Kiefergelenks; 5.Auflage.

Georg Thieme Verlag, Stuttgart 2000

\section{Schwenzer N, Ehrenfeld M:}

Zahnärztliche Chirurgie.

Thieme, Stuttgart/New York 2009

Song F, O'Meara S, Wilson P, Golder S, Kleijnen J (2000):

The effectiveness and cost-effectivness of prophylactic removal of wisdom teeth. Health Technol Assess 4, 1-55

\section{Strietzel FP, Reichart PA (2002):}

Wundheilung nach operativer Weisheitszahnentfernung.

Mund Kiefer GesichtsChir $\underline{6}, 74-84$

\section{Suarez-Cunqueiro MM, Gutwald R, Reichman J, Otero-Cepeda XL,} Schmelzeisen R (2003):

Marginal flap versus paramarginal flap in impacted third molar surgery: a prospective study.

Oral Surg Oral Med Oral Pathol Oral Radiol Endod 95, 403-408 
Taschieri S, Del Fabbro M, Testori T, Weinstein R (2008):

Microscope versus endoscope in root-end managment: a randomized controlled study.

Int J Oral Maxillofac Surg $\underline{37}, 1022-1026$

\section{Ten Cate A:}

Oral Histology: Development, structure, and function.

Mosby Elsevier, St. Louis Missouri 1998.

Tolstunov L, Javid B, Keyes L, Nattestad A (2011):

Pericoronal ostectomy: an alternative surgical technique for management of mandibular third molar in close proximity to the inferior alveolar nerv.

J Oral Maxillofac Surg $\underline{69}$, 1858-1866

Valmaseda-Castellon E, Berini-Aytes L, Gay-Escoda C (2000):

Lingual nerv damage after third lower molar surgical extraction.

Oral Surg Oral Med Oral Pathol Oral Radiol Endod 90, 567-573

Van Gool AV, Ten Bosch JJ, Boering G (1977):

Clinical consequences of complaints and complications after removal of the mandibular third molar.

Int J Oral Surg $\underline{6}, 29-37$

Von Arx T, Frei C, Bornstein MM (2003):

Periradikular surgery with and without endoscopy: a clinical and prospective study.

Schweiz Monatsschr Zahnmed 113, 860-865

\section{Von Wowern N, Nielsen HO (1989):}

The fate of impacted lower third molar after the age of 20 : a four year clinical followup.

Int J Oral Maxillofac Surg $\underline{18,277-280}$

\section{Wagner W, Kunkel M, Koch FP:}

Zahnentfernung; in Zahnärztliche Chirurgie; hrsg. v. Schwenzer N, Ehrenfeld M, Thieme-Verlag, Stuttgart/NewYork 2009, 24

\section{Ward T (1956):}

The split bone technique for removal of lower third molar.

Br Dent J 101, 297-304

Weber T :

Memorix-Zahnmedizin.

Thieme-Verlag, Stuttgart 2000 


\section{Williams PL:}

Gray`s anatomy. The anatomical basis of medicine and surgery.

Churchill Livingstone, Edinburgh London New York

Philadelphia Sydney Toronto 1995, 1240-1241

\section{Winter G:}

Principles of exodontia an applied to the impacted mandibular third molar.

American Medical Book Co, St. Louis 1926 


\section{Abbildungsverzeichnis}

Abb.1.1 Röntgenologische Darstellung der Verlagerungsformen, die Klassifikation ist nach Winter (1926)

Abb.1.2 Schematische Darstellung der Pell- und Gregory- Klassifikation nach kranio-kaudaler Lage

Abb.1.3 Schematische Darstellung der Pell- und Gregory- Klassifikation nach Lagebeziehung zum aufsteigenden Unterkieferast

Abb.1.4

Bildliche Darstellung der Komma-Inzision

Abb.1.5 Bildliche Darstellung der Flapless-Odontotomie: Trennung des Weisheitszahnes ohne vorangegangene Inzision

Abb.1.6

Bildliche Darstellung der lingualen Split-Technik

Abb.1.7

Bildliche Darstellung der Koronektomie

Abb.2.1

Abb.2.2

a) Storz-Hopkins-Optik mit Stütz-Spülschaft

b) Verschiedene Stütz-Spülschäfte zur Aufnahme der Optik

Darstellung des Mikroskops im Operationssaal

Abb.2.3 a und b, Inzision ohne Bildung eines großflächigen Mukoperiostlappens (Mini-Flap-Inzision) beim vollretinierten Weisheitszahn

Abb.2.4 Exposition der Knochenoberfläche, a) Schematische Darstellung b) Mikroskopische Aufnahme

Abb.2.5

Mikrochirurgische Visualisierung des Operationsfeldes

Abb.2.6

Okklusale Exposition, a) Schematische Darstellung

b) Mikroskopische Aufnahme

Abb.2.7 Phase 1-Raumschaffende Trepanation, a) Schematische Darstellung der raumschaffenden Trepanation b) Endoskopische Aufnahme der raumschaffenden Trepanation

Abb.2.8 Phase 2 - Odontosektion, a) Schematische Darstellung

b) Endoskopische Aufnahme

Abb.2.9 Phase 3 - Wurzelentfernung, a) Schematische Darstellung

b) Endoskopische Aufnahme

Abb.2.10 Phase 4 - Alveolenmessung, a) Schematische Darstellung der bukkalen

Knochenhöhenmessung b) Endoskopische Aufnahme der bukkalen

Knochenhöhenmessung

Abb.2.11 Protokoll zur Dokumentation und Bewertung der Operationsaufzeichnung

Abb.2.12 Röntgenologische Messung am (a) vertikalen und (b) horizontalen Weisheitszahn

Abb.2.13 Schematische Darstellung der intraoralen Vermessung des Knochendefektes nach Entfernung des Weisheitszahnes 
Abb.2.14 Schematische Darstellung der Fehlerfaktoren die durch geometrische Umstellungen in der Vermessung verursacht werden konnten

Abb.2.15 Schematische Darstellung der leeren Alveole im Sagittalschnitt

Abb.3.1 Schematische Darstellung der Geschlechtsverteilung und Zahnverteilung im Unterkiefer

Abb.3.2 Box-Plot Darstellung der Altersverteilung vom Patientenkollektiv

Abb.3.3 Grafische Darstellung der Angulationsverteilung mittels Klassifikation nach Winter (1926)

Abb.3.4 Schematische Darstellung der Anwendung von Mikroskop und Endoskop in den vier Phasen

Abb.3.5 Box-Plot Darstellung der Zeitverteilung in den vier Phasen $\quad \mathbf{S . 5 4}$

Abb.3.6 Box-Plot Darstellung der OP-Gesamtdauer $\quad \mathbf{5 . 5 4}$

Abb.3.7 Grafische Darstellung der Angulation im Zusammenhang mit der Operationszeit

Abb.3.8 Box-Plot Darstellung der Knochendefektsituation der bukkalen Alveolenwand prä- und postoperativ

Abb.3.9 Schematische Darstellung der Knochenverlustsituation der bukkalen Alveolenwand prä- und postoperativ: Vergleich aller vermessenen Weisheitszähne

Abb.3.10 Grafische Darstellung des Knochenverlustes im Zusammenhang mit den Angulationstypen

Abb.3.11 Schematische Darstellung der postoperativen Komplikationen

Abb.3.12 Schematische Darstellung der Komplikationen im Zusammenhang mit der Op-Zeit

\section{Tabellenverzeichnis}

Tab.3.1 Tabellarische Darstellung der optischen Bewertung von anatomischen Strukturen

Tab.3.2 Tabellarische Darstellung der Operationszeiten

Tab.3.3 Tabellarische Darstellung der Zähne die eine enge Beziehung zum Nervus alveolaris inferior aufweisen 


\section{Anhang: Patiententabelle}

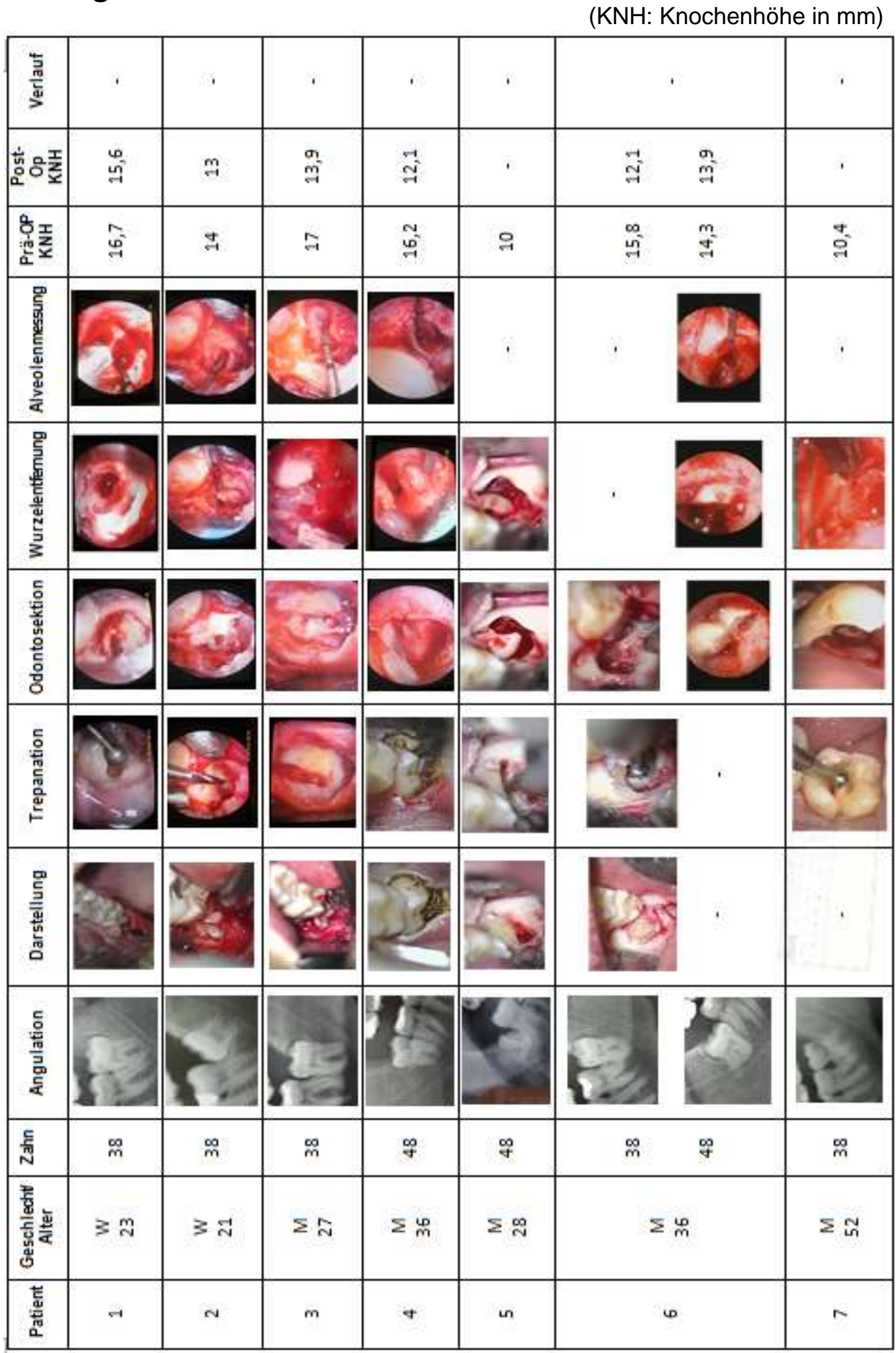




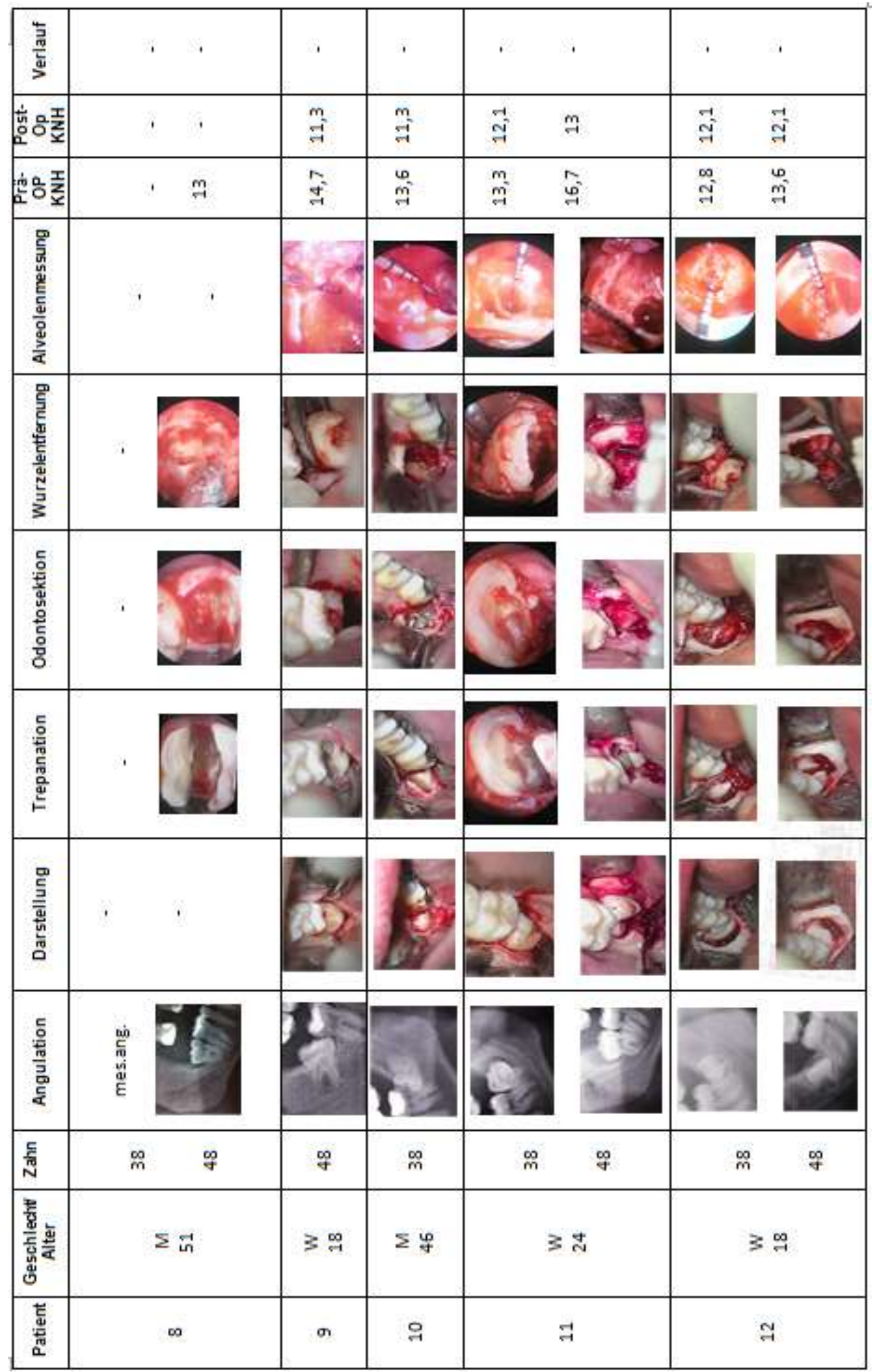




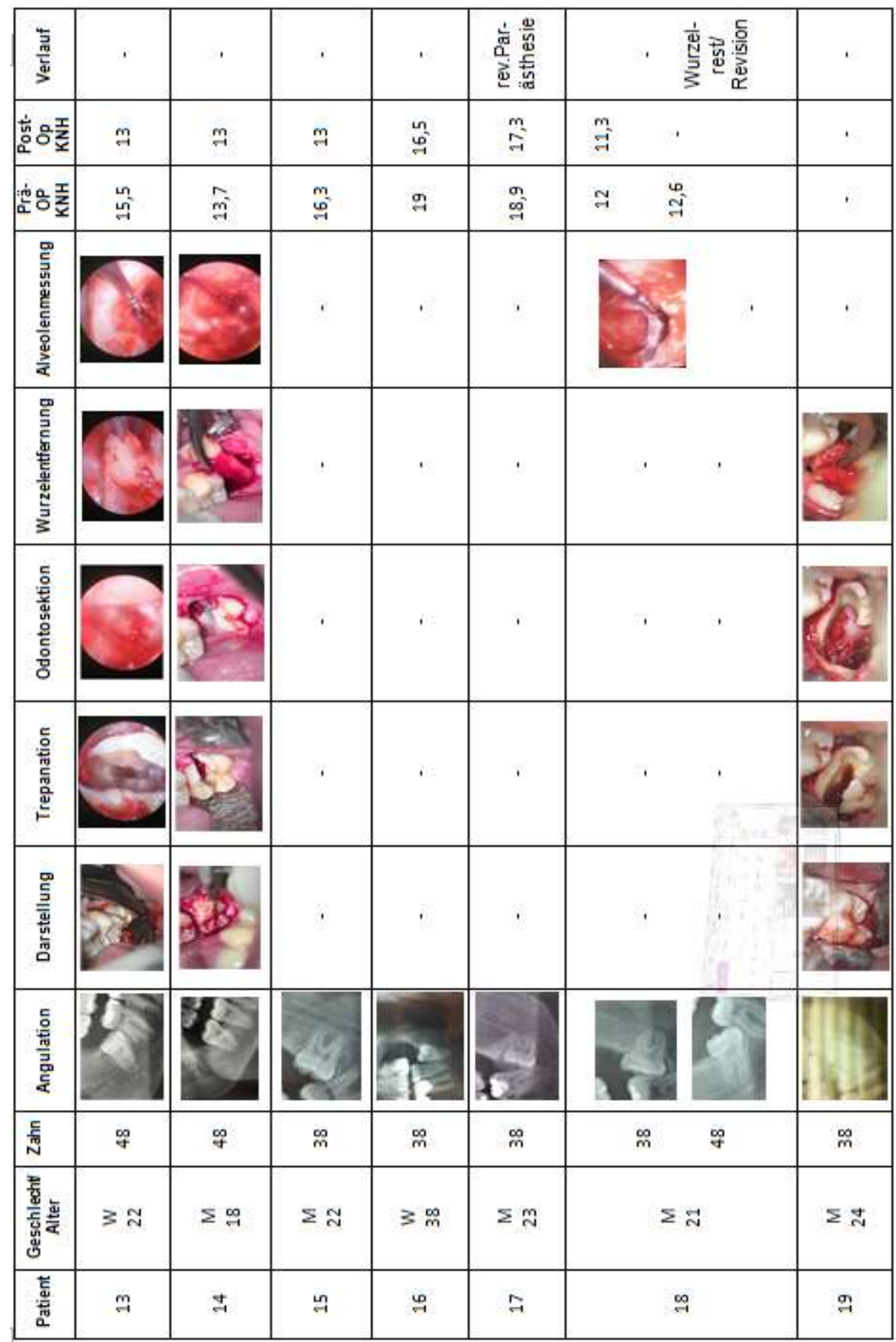




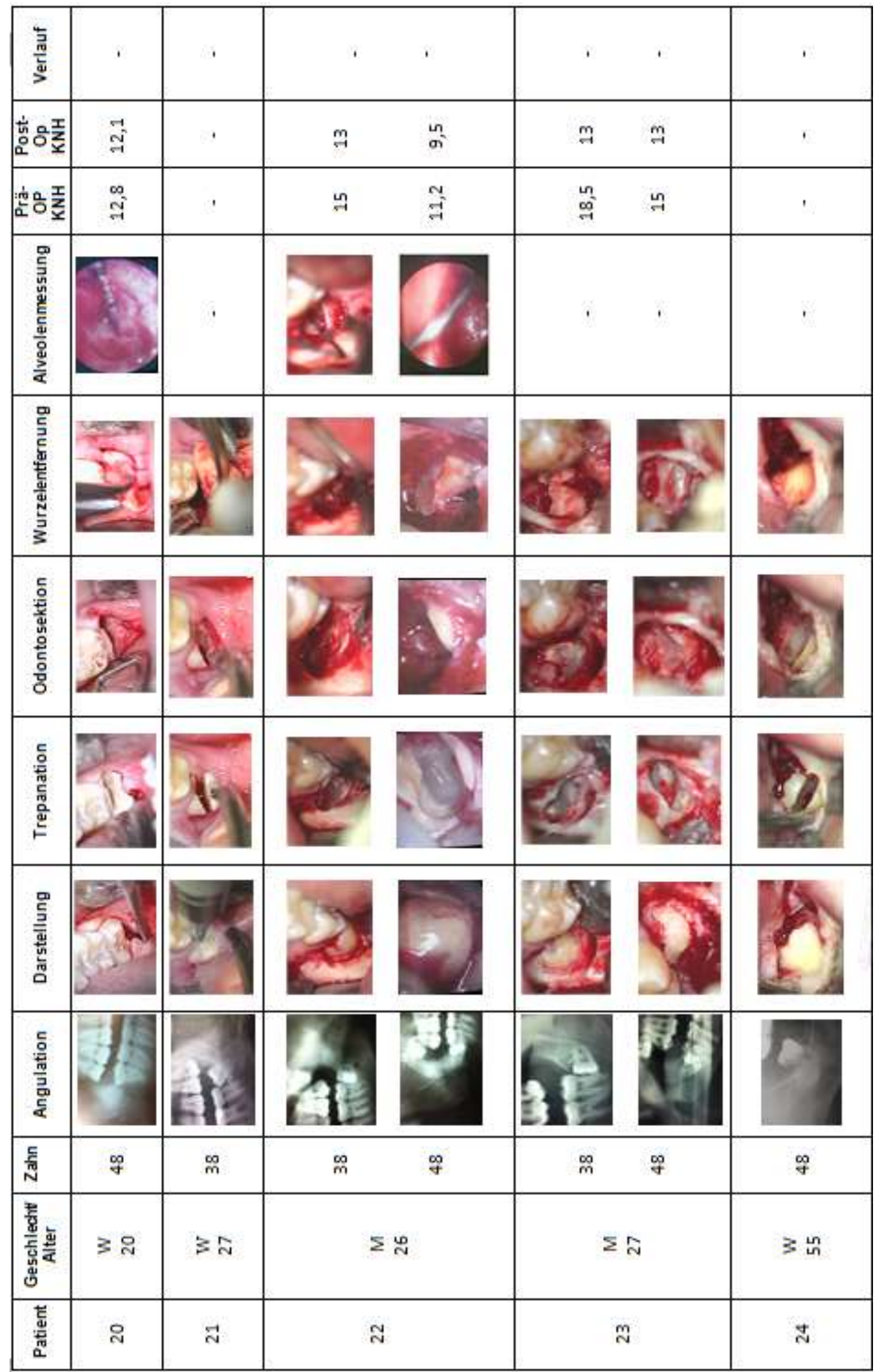




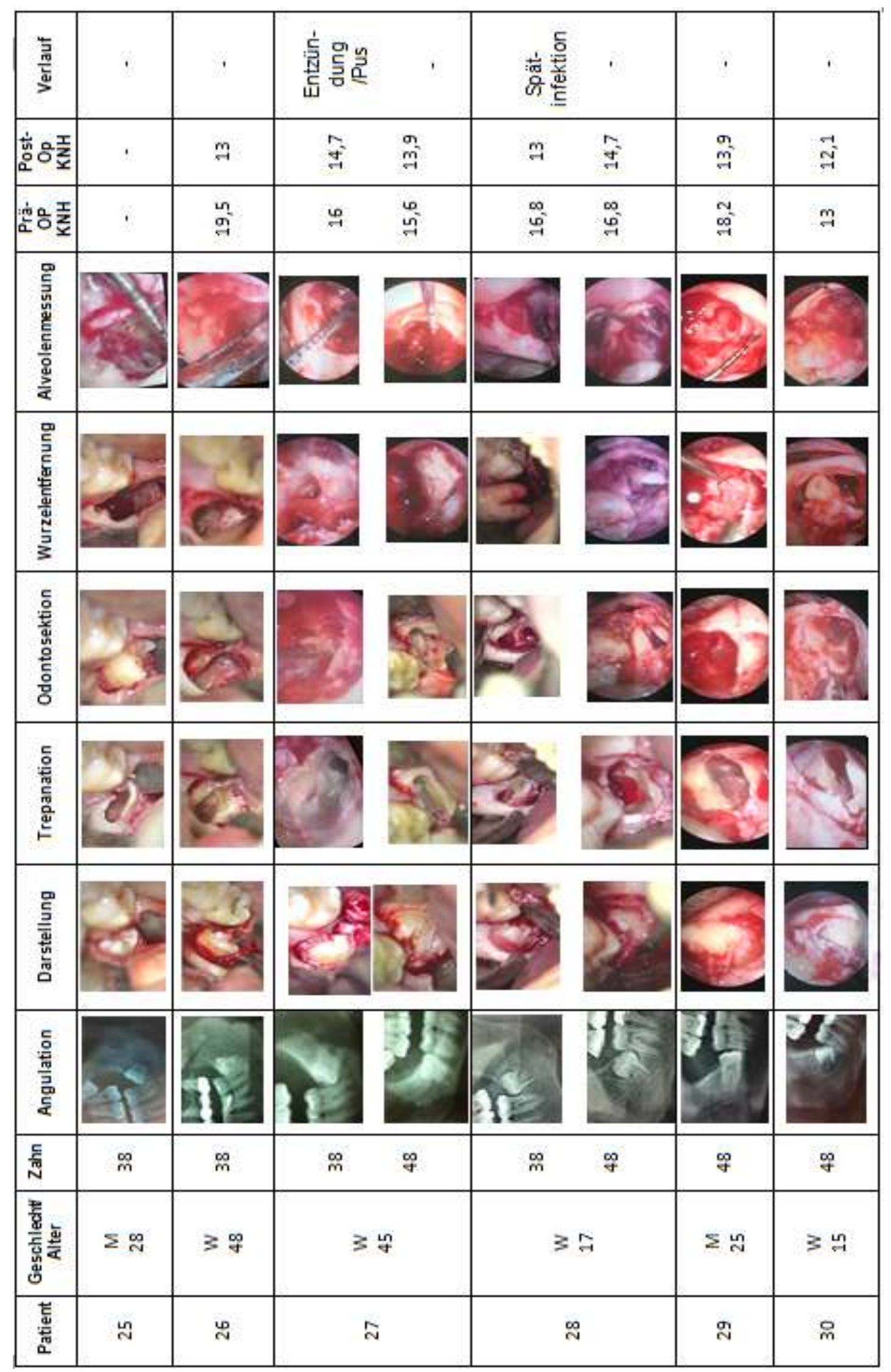




\begin{tabular}{|c|c|c|c|c|c|c|c|c|}
\hline $\begin{array}{l}\frac{5}{3} \\
\text { 竞 } \\
>\end{array}$ & ' & . & 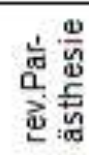 & 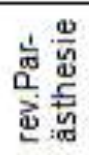 & . & ' & . & ' \\
\hline ثั่ & $\begin{array}{l}0 \\
\text { ले }\end{array}$ & 국 & $\begin{array}{l}\text { ㄱ. } \\
\text { ले }\end{array}$ & $\begin{array}{l}\stackrel{\sigma}{1} \\
\text { ले }\end{array}$ & ' & 9 & 等 & ${ }^{\circ}$ \\
\hline 我을 $\frac{1}{2}$ & 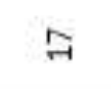 & $\stackrel{\oplus}{-1}$ & $\stackrel{n}{\tilde{F}}$ & 凋 & $\stackrel{n}{7}$ & $\underset{F}{ \pm}$ & $\stackrel{\circ}{\stackrel{0}{\sigma}}$ & $\stackrel{9}{-1}$ \\
\hline 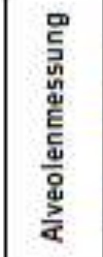 & & & & & ' & & . & . \\
\hline 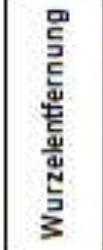 & & & & & & ' & 8 & ' \\
\hline 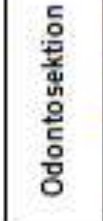 & & & & & & & & ' \\
\hline 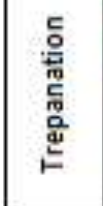 & & & & & & & 28 & ' \\
\hline 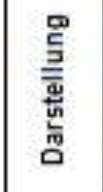 & & & & & & & ' & . \\
\hline 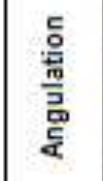 & & & & & & & & 8 \\
\hline \begin{tabular}{|l|} 
岳 \\
N
\end{tabular} & 喿 & $\stackrel{\circ}{m}$ & & 喿 & 哭 & $\stackrel{m}{m}$ & go & $\stackrel{\infty}{\infty}$ \\
\hline 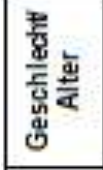 & $3 \stackrel{\circ}{\circ}$ & $3 \pi$ & & & उ & 32 & $\Sigma \bar{d}$ & 3 ิㅗ \\
\hline 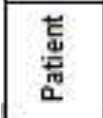 & $\vec{m}$ & लै & & & జా & 菂 & ๓ & $\tilde{\omega}$ \\
\hline
\end{tabular}




\begin{tabular}{|c|c|c|c|}
\hline $\begin{array}{l}\text { 莺 } \\
\text { ऐ. }\end{array}$ & ' & ' & , \\
\hline 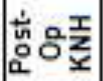 & 국 & 9 & $\underset{\infty}{n}$ \\
\hline 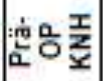 & 売 & 哭 & $\frac{n}{7}$ \\
\hline 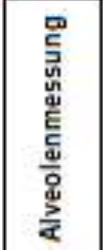 & ' & . & , \\
\hline 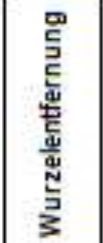 & ' & ' & . \\
\hline 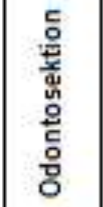 & ' & 1 & . \\
\hline 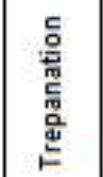 & . & . & . \\
\hline 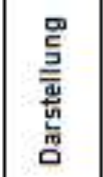 & ' & ' & , \\
\hline 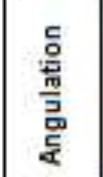 & & & 8 \\
\hline 熺 & 舫 & $\stackrel{\infty}{\infty}$ & क्ष \\
\hline 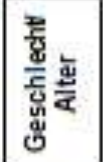 & 39 & $\Sigma 8$ & $\Sigma \bar{N}$ \\
\hline 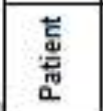 & ஜ & 이 & 웡 \\
\hline
\end{tabular}




\section{Danksagung}

An dieser Stelle möchte ich mich ganz herzlich bei Herrn Prof. Dr. Dr. W. Engelke für die freundliche Überlassung des Themas und für seine hilfreiche Unterstützung dessen Bearbeitung bedanken.

Bedanken möchte ich mich auch bei Herrn S. Schneider aus der Abteilung medizinische Statistik, für die geleistete Unterstützung bei der statistischen Auswertung der Untersuchungsergebnisse dieser Arbeit.

Für die interessanten wissenschaftlichen Anregungen wie auch Korrektur dieser Arbeit bedanke ich mich auch bei meinen Kollegen J. Kupis und M. Müller. 


\section{Lebenslauf}

Ich Eun-Jin Choi, bin am 19.04.1985 in Aachen geboren und bin Südkoreanerin. Meine Eltern sind Sung-In Choi geboren am 23.02.1954 in Korea und Kyung-Ok Choi (geb. Lee) geboren am 07.07.1960 ebenfalls in Korea. Ich habe noch zwei Schwestern.

Ich besuchte von 1991-1995 die Glückaufgrundschule in Siegen und wanderte 1995 mit der Familie nach Korea aus. Dort besuchte ich weiterhin noch 2 Jahre die Koreanische Grundschule. Von 1998-2001 besuchte ich die Jung-Ja Middle School und von 2001-2004 die Imae High School. Die allgemeine Hochschulreife erwarb ich in Korea 2003.

Im Sommer 2004 kam ich nach Deutschland, besuchte für 8 Monate einen Deutschkurs in Hamburg und von 2005-2010 studierte ich an der Georg-AugustUniversität Göttingen Zahnmedizin.

Seit Oktober 2011 arbeite ich als wissenschaftliche Mitarbeiterin in der Abteilung Präventive Zahnmedizin, Parodontologie und Kariologie an der Universitätsmedizin Göttingen. 